\section{Surface Chemistry of Catalysis by Gold*}

\author{
R. Meyer*, C. Lemire, Sh. K. Shaikhutdinov, \\ H.-J. Freund \\ Department of Chemical Physics, Fritz-Haber-Institute \\ der Max-Plank-Gesellschaft, Faradayweg 4-6, 14195 \\ Berlin, Germany
}

\section{Introduction}

Gold has long been regarded as an "inert" surface and bulk gold surfaces do not chemisorb many molecules easily. However, in the last decade, largely through the efforts of Masatake Haruta, gold particles, particularly those below $5 \mathrm{~nm}$ in size, have begun to garner attention for unique catalytic properties (1-8). In recent years, supported gold particles have been shown to be effective as catalysts for low temperature CO oxidation (9), selective oxidation of propene to propene oxide (10), water gas shift (11), NO reduction (12), selective hydrogenation of acetylene (or butadiene) (13) and other reactions (1-5,14-16). Currently used in Japanese toilets for odour reduction (3), gold has demonstrated industrial potential as well for the hydrochlorination of ethyne to vinyl chloride (16-18) and as a bimetallic component of vinyl acetate monomer production catalysts $(19,20)$. Low temperature CO oxidation is of particular importance, finding applications in indoor air quality applications (21) and as a guard bed catalyst to prevent $\mathrm{CO}$ poisoning of proton exchange membrane fuel cells (22-24).

${ }^{*}$ This review is an expansion of the Keynote lecture given by Prof. Freund at Gold 2003
Numerous experimental and theoretical studies are currently being performed which are aimed at understanding why gold starts to catalyse these reactions when its dimensions go to a nanometer scale. The recent surge in study of gold catalysts, which has been reviewed by Haruta (1-4), Bond (57) and Thompson $(5,6,8)$, has been accompanied by a corresponding swell of interest in the surface science of gold. This review will not attempt to be completely comprehensive but rather to give the reader a view of the current state of research in the attempts to understand some of the unique catalytic chemistry of gold. Of particular note, the electrochemistry of gold and the formation of self assembled monolayers of various organics (particularly thiols) are subjects that demand their own separate reviews and the authors will not present a discussion of these areas. The review will focus on issues in heterogeneous chemistry, although gold has proven to have applications in homogeneous catalysis as well $(8,16,25,26)$. In general, surface science studies attempt to relate properties of welldefined model structures to more complex systems. Therefore both results from gold single crystals and more intricate systems involving deposited gold particles will be presented with a discussion of their relevance to real catalysts.

Although the catalytic nature of gold may be linked to size and structure issues, it seems appropriate to begin any discussion with some general observations about gold's unique chemistry. Due to the large size of its nucleus, relativistic effects become critically important to an evaluation of gold chemistry (27-30). In short, the s orbitals and to a lesser extent the $p$ orbitals are found to contract in response to the large nuclear charge, while the $d$ orbitals (and $f$ orbitals) are in fact expanded. In the case for gold, the energy difference between the $6 s$ and $5 d$ orbitals shrinks by over $60 \%$ (28) when compared to the theoretically modeled non-relativistic case. Calculations reveal that these effects experience a local maximum for gold when examining its place in the Periodic Table (29). Consequently, the $5 d$ band becomes a focal point of gold chemistry and gold displays many extraordinary characteristics. For example, the higher cohesive energy of gold as compared to silver can be explained by the increased participation of the $d$ orbitals in metal-metal bonding. Gold also has the highest electronegativity of any metal and is only slightly more electropositive than such non-metals as sulfur and iodine. Gold possesses an extremely high electron affinity (2.31 eV) and ionization potential (9.22 eV) as well (30). Gold is the only transition metal which does not have a stable oxide $\left(\mathrm{Au}_{2} \mathrm{O}_{3}\right.$ is only metastable) (31). Gold also possesses a unique ability to interact with itself, known as aurophilic bonding, which allows for the formation of superstructures of gold containing molecules (32). As we will discuss later, relativistic 
effects continue to be important even when shrinking the size of gold and/or changing its chemical environment $(27,33,34)$.

This review is organized as follows. First, we begin with the structure and adsorption properties of Au crystalline surfaces. Next, we will discuss reactions that have been observed on gold single crystals, focusing on the oxidation of CO using oxygen atoms. We will also briefly discuss the use of gold in bi-metallic systems and the contribution to this field made by surface science. Thereafter, we will examine lessons learned from gas phase experiments before moving on to theoretical predictions for the adsorption and reaction properties of gold single crystal surfaces and free gold clusters. Then, we will discuss model catalyst systems, focusing on the relationship between the support and gold with regard to structure and reactivity. Lastly, we will discuss the unresolved questions surrounding gold raised by the catalytic community and attempt to frame how surface science can help answer such questions.

Most common abbreviations used in surface science and related experimental techniques can be found in Table 1. A further point of clarification is that for the purposes of this review, "clusters" are defined to be particles with a precise number of atoms.

\section{Gold single crystal chemistry \\ 2.1 Surface structures of Au single crystals}

The low Miller indexed surfaces, (111), (110) and (100), of gold, depicted in Fig. 1a-c, are all known to undergo reconstruction. Gold is the only element with a face centered cubic (fcc) crystalline structure whose (111) face reconstructs under UHV conditions (36). The reconstruction can be described by a complex stacking-fault-domain model by which both fcc and hcp domains are present on the surface with narrow transition regions in between them. The reconstructed surface, which is sometimes referred to as a $(23 \times \sqrt{3})$ structure $(37)$, has been examined in much detail by LEED (37), helium atom scattering (38) and STM (36). The unit cell of $63 \times 4.7 \AA$ experiences contraction in the $\{1-10\}$ direction, as 23 atoms sit on top of 22 hollow sites. The transition domains between regions of

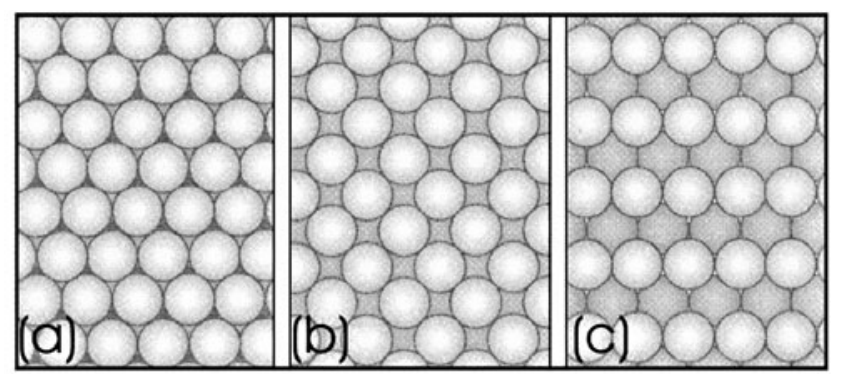

Figure 1

The (a) (111), (b) (100) and (c) (110) fcc crystal surfaces. (35)
Table 1

Useful abbreviations found in the text

\begin{tabular}{|c|c|}
\hline Abbreviation & Technique \\
\hline AES & Auger electron spectroscopy \\
\hline AFM & Atomic force microscopy \\
\hline DOS & Density of states \\
\hline DFT & Density functional theory \\
\hline DRIFTS & $\begin{array}{l}\text { Diffuse reflectance Infrared transmission } \\
\text { spectroscopy }\end{array}$ \\
\hline EELS & Electron energy loss spectroscopy \\
\hline EID & Electron induced decomposition \\
\hline EPR & Electron paramagnetic resonance \\
\hline EXAFS & Extended $\mathrm{x}$-ray absorption fine structure \\
\hline FTIR & Fourier transform infrared spectroscopy \\
\hline HREELS & $\begin{array}{l}\text { High resolution electron energy loss } \\
\text { spectroscopy }\end{array}$ \\
\hline HREM & High-resolution electron microscopy \\
\hline HRSEM & $\begin{array}{l}\text { High resolution scanning electron } \\
\text { microscopy }\end{array}$ \\
\hline HOMO & Highest occupied molecular orbital \\
\hline IRAS & $\begin{array}{l}\text { Infrared reflection absorption } \\
\text { spectroscopy }\end{array}$ \\
\hline LEED & Low energy electron diffraction \\
\hline LEIS & Low energy ion spectroscopy \\
\hline LUMO & Lowest unoccupied molecular orbital \\
\hline SEM & Scanning electron microscopy \\
\hline STM & Scanning tunneling microscopy \\
\hline TEM & Transmission electron microscopy \\
\hline TPD & Temperature programmed desorption \\
\hline TPR & Temperature programmed reaction \\
\hline UHV & Ultra high vacuum \\
\hline UPS & Ultraviolet photoemission spectroscopy \\
\hline XANES & X-ray absorption near-edge structure \\
\hline XPS & X-ray photoelectron spectrocopy \\
\hline XRD & X-ray diffraction \\
\hline $\mathrm{ML}$ & Monolayer coverage \\
\hline L & Langmuir, $10^{-6}$ torr-sec \\
\hline
\end{tabular}

different stacking configurations were imaged as pairs of parallel lines along the $\{11-2\}$ direction. A surface corrugation of $0.2 \AA$ was measured by STM between the high point (transition lines) and low point (fcc minima) on the reconstructed terraces. Transition lines were found to come together occasionally to form closed off $U$-structures with the fcc region enclosed by the $U$ and the hcp region left outside as shown in the inset of Fig 2. 


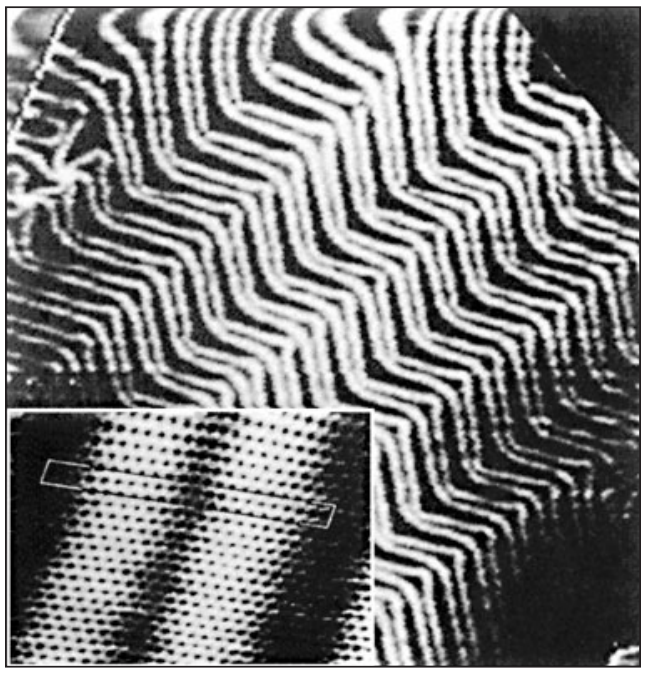

Figure 2

STM image of the reconstructed Au (111) surface (size $120 \times 120 \mathrm{~nm}^{2}$ ). Inset $\left(8 \times 6 \mathrm{~nm}^{2}\right)$ shows an atomic resolution image with the unit cell indicated. (36)

Large-scale images, as presented in Fig. 2, revealed a zigzag pattern (named the herringbone pattern) that results from joining of $120^{\circ}$ rotated domains. The surface reconstruction of $\mathrm{Au}(111)$, while unique to fcc pure metals, is actually similar to that found when depositing a metal adlayer on top of another metal substrate with a slightly larger atomic spacing (39). The reconstruction of gold can be rationalized in terms of unique surface states that arise due to the interaction of $s p$ and $d$ states, a consequence of its relativistic nature (40). X-ray scattering measurements indicate that the reconstruction is stable up to $865 \mathrm{~K}$, whereupon long range order is lost (41). Although the reconstruction is stable in air at room temperature (42), it can be lifted upon adsorption of certain gases. For example, exposure of above 500 mbar $\mathrm{CO}$ at $300 \mathrm{~K}$ results in an expansion of the surface layer such that it is partially returned to the unreconstructed $\mathrm{Au}(111)$ (43). In their examination of the stability of the reconstruction, Ertl and co-workers observed that the distance between the stacking fault lines shrank continuously with increasing potassium coverage (44). At coverages above $0.2 \mathrm{ML}$, the surface formed a new structure whereby the density of surface atoms was increased. This was attributed to an adsorbate induced weakening of bonds between the first and second layer and a simultaneous increase in bonding among surface atoms. Atomic oxygen has also been used to alter the (111) surface structure such that a $(\sqrt{3} \times \sqrt{3}) \mathrm{R} 30^{\circ}$ structure evolves (4549). This surface can be reduced back to the herringbone pattern described above when the sample is exposed to a reducing agent such as methanol or hydrogen (49). However, the extreme conditions under which these restructurings take place (several hours at over $800^{\circ} \mathrm{C}$ and 1 bar $\mathrm{O}_{2}$ ) suggest

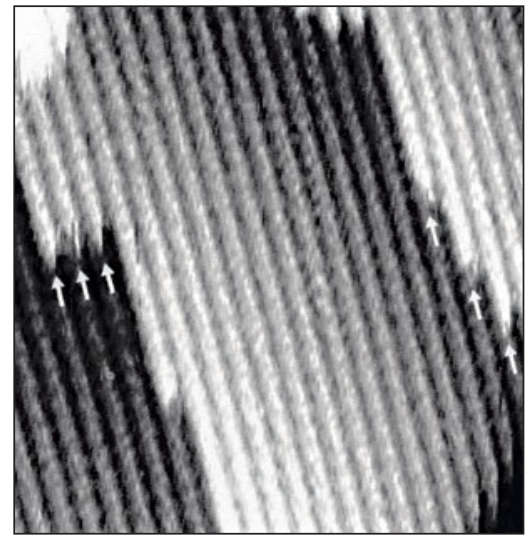

Figure 3

STM image of a "missing row" reconstruction of the $A u(110)-(1 \times 2)$ surface (size $15 \times 15 \mathrm{~nm}^{2}$ ). (56)

that such surfaces are not appropriate models for catalytically active systems.

As first observed by Fedak and Gjostein (50), the Au(110) surface reconstructs into the $(1 \times 2)$ surface which is formed by "missing row" along $\{1-10\}$ direction which is also observed for $\operatorname{Ir}(110)$ (51) and Pt(110) (52) and shown in Figure 3. The missing row reconstruction gives rise to surface atoms with three different coordination numbers: on top of row atoms, side of row atoms, and trench atoms. The adsorption behavior of $\mathrm{fcc}(110)$ surfaces is often considered in terms of different types of sites relating to the coordination number (53). The sides of row atoms give rise to a site similar to a three-fold hollow site on an $\mathrm{fcc}(111)$ metal and are sometimes referred to as (111) microfacets. Although this structure has been observed by LEED (54), X-ray diffraction (55) and STM $(53,56)$, the exact details are still not entirely known. The top layer spacing is estimated to be contracted by up to $20 \%$ in the vertical direction (57). The reconstruction affects not only interatomic spacings in the surface layer but also the second layer and third layers experience compensating movements both laterally and vertically. As mentioned above, the missing row reconstruction is observed for the $5 d$ metals but not their $4 d$ counterparts. This suggests that the reconstruction is stabilized by the interactions involving more delocalized $5 d$ electrons (58). The surface has been previously described to undergo an order-disorder transition at above $700 \mathrm{~K}$ (59). STM experiments have shown this is actually a two-step process as the reconstructed surface is stable in UHV up to $\sim 650 \mathrm{~K}$ whereupon two-dimensional roughening occurs adjacent to step edges (60). Further three-dimensional roughening concomitant to surface deconstruction occurs upwards of 700 K. In an examination of CO adsorption on defects, Gottfried et al. found that defects on Au(110)-(1x2) induced by $\mathrm{Ar}^{+}$ion bombardment were completely healed by heating to $500 \mathrm{~K}$, thus showing the high mobility of surface atoms at relatively low temperatures (61). 


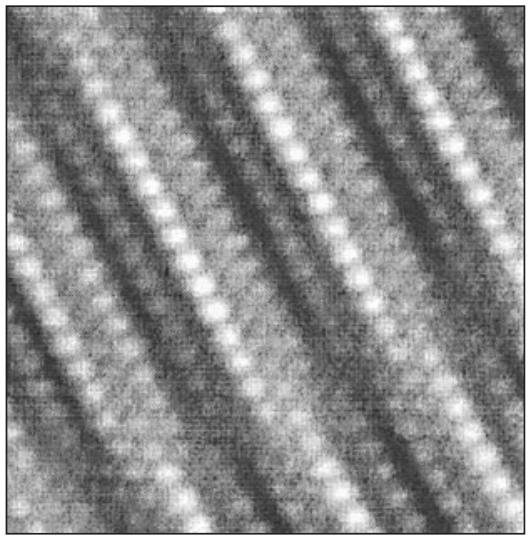

Figure 4

High resolution STM image of the reconstructed Au (100)-(1x5) surface (size $\left.5.3 \times 5.3 \mathrm{~nm}^{2}\right) .(62)$

Perhaps the most complicated of the three low indexed surfaces, the reconstruction of the (100) surface of gold is a subject of significant controversy. In Figure 4, a high resolution STM image reveals significant surface corrugation that seems to agree well with the originally suggested $(5 \times 1)$ reconstruction (50) where the added rows run along the $\{100\}$ direction. Since that time, various models have been put forward, and then later a $(5 \times 20)(63)$, a $(5 \times 20)$ with

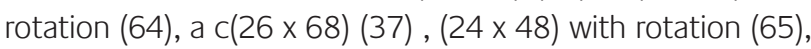
and a hexagonal $(28 \times 5)$ R0.6 ${ }^{\circ}(66)$.

It has even been proposed that the reconstructed surfaces may experience different combinations of these structures depending upon step density and surface temperature (67). The reconstruction is generally believed to be limited to the first layer, thus indicating that the more compact surface arrangement is favored to a degree where the energy cost due to a lack of commensuration with the layer underneath can be overcome (68). However, Rieder et al using electron density calculations found that the best fit to their Hediffraction data occurred when they allowed for small changes in the interatomic spacing of the lower layers as well (69). The enhancement of surface bonding was seen by Annett and Inglesfield to arise as a consequence of surface band narrowing (70). Calculations by Takeuchi et al. interpreted the reconstruction primarily in terms of a relativistic effect manifested by the high degree of $d$ orbital participation in bonding, as was the case for $\mathrm{Au}(111)(71,72)$. Marks and Smith proposed that the reconstruction is actually the result of surface Shockley partial dislocations (73). When the unreconstructed surface is prepared, HREM images revealed that small displacements could be seen such that one whole column of atoms appeared to be shifted both laterally and away from the surface. Upon heating to $373 \mathrm{~K}$ the surface transforms back to the more stable reconstructed surface (74). Marks and Smith asserted that the large degree of mass transfer that must take place for the reconstruction must occur through a mechanism involving the observed dislocations (73). X-ray scattering measurements indicated that above $1170 \mathrm{~K}$ the reconstruction is lifted and only the (1 $x 1$ ) phase of the bulk can be seen, thus implying the surface layer has undergone an order-disorder transition $(75,76)$. In a similar way to the adsorption behavior of potassium on $\mathrm{Au}(111)$, at coverages of about $0.1 \mathrm{ML}$ the reconstruction vanishes (77,78). As the coverage increases above $0.25 \mathrm{ML}$, a (1x2) reconstruction, similar to the (110) surface discussed above, forms.

\subsection{Adsorption on Au single crystals}

As with other metals, the adsorption of $\mathrm{CO}$ on gold has been extensively studied. Early work of Trapnell on gold films showed that adsorption of CO on gold is weak compared to most other metals and the heat of adsorption of $\mathrm{CO}$ on gold was estimated to be $8.7 \mathrm{kcal} / \mathrm{mol}$ (79). In their investigation of gold-platinum alloy films, Stephan and Ponec, observed two desorption states for $\mathrm{CO}$, at $110 \mathrm{~K}$ and a shoulder at 170 $\mathrm{K}$ which they assigned to gold (80). In Bradshaw and Pritchard's examination of CO on a rough gold film, they also observed a slight red shift in the IR spectra with increasing coverage (81). An adsorption isotherm at $113 \mathrm{~K}$ revealed linear behavior, indicating that despite the film's roughness, CO appeared to adsorb on only a single type of adsorption site. Dumas et al (82) examined CO adsorption on rough and annealed gold films. They found that while on smooth films no CO adsorption was observable by IR spectroscopy even at $25 \mathrm{~K}$, on rough films an adsorption state existed at $2125 \mathrm{~cm}^{-1}$ up to $170 \mathrm{~K}$. In contrast to the adsorption behavior of $\mathrm{CO}$ on many other metals, a small shift toward lower wave numbers

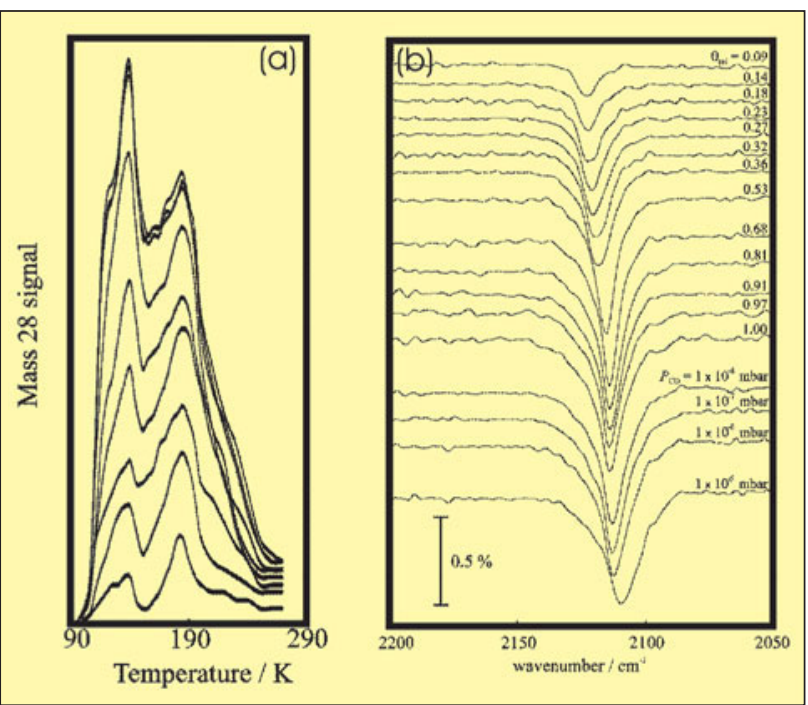

\section{Figure 5}

(a) Temperature programmed desorption spectra following $\mathrm{CO}$ exposure of $\mathrm{Au}(332)$ at $92 \mathrm{~K}$ as a function of CO coverage. (b) FTIR spectra of CO adsorption on Au(332) as a function of coverage. $(83,84)$ 
with increasing CO coverage was observed. In Ruggerio and Hollins' work on Au(332) $(83,84), 3$ species were identified by reflection absorption infrared spectroscopy (RAIRS), including a central peak that shifted from $2124 \mathrm{~cm}^{-1}$ to $2110 \mathrm{~cm}^{-1}$ as coverage increases, with both a red shifted shoulder and a blue shifted shoulder as shown in Fig 5b. Interestingly, the corresponding TPD spectra shown in Fig 5a revealed only two main peaks, one at about $140 \mathrm{~K}$, the second at $185 \mathrm{~K}$.

More recently, Gottfried et al. have examined the adsorption of $\mathrm{CO}$ on $\mathrm{Au}(110)-(1 \times 2)$ in great detail (61). Five separate adsorption states were identified: $\alpha$ (multilayer at $32 \mathrm{~K}$ ), $\beta$ (2nd layer at $37 \mathrm{~K}$ ), $\delta$ (physisorbed first layer at $55 \mathrm{~K}$ ), $\sigma$ (also physisorbed first layer at $67 \mathrm{~K}$ ), and $\epsilon$ (chemisorbed at $145 \mathrm{~K})$. No ordered overlayer structures were detected with LEED for any coverage examined. CO did not lift the $(1 \times 2)$ reconstruction as has been seen in the case of CO on Pt (110) $(85,86)$. The $\epsilon$ peak was found to saturate at about $0.13 \mathrm{ML}$, a much lower saturation coverage when compared to CO adsorption on other metals. As Outka and Madix failed to detect $\mathrm{CO}$ adsorption at temperatures down to $125 \mathrm{~K}$ on $\mathrm{Au}(110)$, one could conclude that the $\epsilon$ feature is associated with defects (87). However, after ion bombardment, a new peak formed at $190 \mathrm{~K}$ with an additional shoulder extending out to $230 \mathrm{~K}$. In addition, ion bombardment did not lead to growth of the $\epsilon$ peak, thus indicating that the $\epsilon$ peak is not related to defects, but rather comes from a regular feature of the $\mathrm{Au}(110)-(1 \times 2)$ surface. Polarization resolved UPS measurements showed that chemisorbed $\mathrm{CO}$ may prefer an orientation parallel to the surface. Further support of a parallel orientation was given by a lack of intensity for the $5 \sigma$ orbital contribution, based upon assignments from the measurement of physisorbed $\mathrm{CO}$. CO was conceived to bond to the surface primarily through back donation from $\mathrm{Au} 5 d$ levels into the $\mathrm{CO} 2 \pi^{*}$ orbital. Previously, UPS experiments had revealed that although the surface was capable of stronger $\mathrm{CO}$ chemisorption on $\mathrm{Au}(110)$, the saturation chemisorbed coverage on $\mathrm{Au}(110)$ was significantly lower (only $0.3 \mathrm{ML}$ ) $(88,89)$.

Goodman's lab determined a slightly lower adsorption energy than that found by Gottfried et al for the low coverage limit on Au(110)-(1x2) (90) (10.9 kcal/mol as compared to $\sim 14 \mathrm{kcal} / \mathrm{mol})$. Similar to Bradshaw and Pritchard (81) and Hollins and Ruggerio (83,84), a red shift in the CO stretching frequency was observed with increasing coverage. This phenomena was interpreted as the combined result of a strong "wall effect" arising from steric repulsion between the CO $5 \sigma$ and the filled $d$ band of gold, a weak $5 \sigma$ donation from $\mathrm{CO}$ to gold and a significant $2 \pi$ backdonation from the metal to $\mathrm{CO}$.

CO adsorption has also been examined on $\mathrm{Au}(100)$ (91). By measuring the surface potential in a CO environment over a range of temperatures, the heat of adsorption was estimated to be $58 \mathrm{~kJ} / \mathrm{mol}$. This value is in agreement with findings by Gottfried et al (59 kJ/mol) for CO on Au(110)-(1×2) (61). As was the case for $A u(110)$, no ordered LEED structure was observable for $\mathrm{CO}$ on $\mathrm{Au}(100)$.

At elevated pressure CO can interact in a stronger manner with gold surfaces. Peters et al found by surface $X$-ray diffraction evidence for lattice expansion of the $\mathrm{Au}(111)$ surface exposed to CO pressures between 0.1-530 mbar at $300 \mathrm{~K}$ (43), although the long range herringbone pattern was preserved. More extensive reconstruction of the $\mathrm{Au}(111)$ surface was observed when exposed to $110 \mathrm{mbar}$ CO at $325^{\circ} \mathrm{C}$. Since the surface remained disordered after evacuation, the authors concluded that dissociative adsorption must have occurred.

Jugnet et al. observed similar behavior for Au(110) for CO pressures above 0.1 mbar (92). STM results showed significant surface roughening and a lifting of terrace anisotropy. This new roughened surface was stable following removal of CO. Furthermore, using polarized IRAS, a single $\mathrm{CO}$ adsorption state at $2110 \mathrm{~cm}^{-1}$ was seen, which the authors tentatively assign to on-top adsorption as seen for other fcc(111) surfaces $(93,94)$. However, as exposure time increased, the signal decreased, indicating possible dissociation of CO on the surface. STM images confirmed that the surface reorganization was accompanied by surface deposits which were believed to be carbon.

Much work has been devoted to exploring the nature of oxygen on gold surfaces in order to better understand the unique oxidation catalysis of gold nanoparticles. However, the surface of gold single crystals cannot be populated with chemisorbed molecular or dissociated oxygen, no matter how large the exposure to molecular oxygen $(79,95)$, except at very high temperatures as described in Comsa's work (4549). Although some early research reported chemisorption of oxygen (96-98), later work has ascribed these results to oxygen dissociation on calcium or silicon impurities $(99,100)$. This has led researchers to employ other means to deliver oxygen to the surface. Canning et al. used a hot filament to crack oxygen and thereby populated a bulk gold sample with atomic oxygen (95). The surface oxide could by decomposed completely by heating and oxygen was observed to desorb associatively in a single desorption peak at $650 \mathrm{~K}$. Similar results were later obtained in the same way for atomic oxygen on $\mathrm{Au}(110)$, yielding a single peak at $590 \mathrm{~K}$ (87).

Koel and co-workers have used ozone to deliver oxygen to $\mathrm{Au}(111)$ (101-103). They observed the formation of a single peak at $550 \mathrm{~K}$ that shifted to slightly lower temperatures for very low coverages. No ordered overlayer for oxygen on $\mathrm{Au}(111)$ was observed by low energy electron diffraction (LEED), and an oxygen coverage as small as 0.1 ML was sufficient to lift the surface reconstruction. Davis and Goodman later confirmed this result for the Au(111) surface 
and found that oxygen desorbs recombinatively in a single peak at $470 \mathrm{~K}$ from $\mathrm{Au}(100)$ (104). Again, no ordered overlayer structure was observed by LEED.

Christmann's group has induced oxygen chemisorption by irradiating a $\mathrm{Au}(110)$ surface covered with physisorbed oxygen with low energy electrons or UV photons at $28 \mathrm{~K}$ $(105,106)$. Oxygen could then be seen to desorb in two peaks, low temperature feature at $497 \mathrm{~K}$, which was assigned

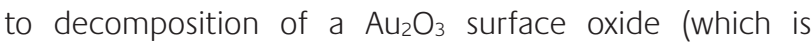
inherently unstable) and a second peak at $553 \mathrm{~K}$ which is assigned to chemisorbed oxygen on the surface. Unlike earlier studies which report first order kinetics, second order kinetics were observed for oxygen desorption, which is typical for associative desorption of a diatomic. Further experiments involving the bombardment of a $\mathrm{Au}(110)$ surface by $\mathrm{O}^{+}$ions showed that four additional states of chemisorbed oxygen could be obtained at $415 \mathrm{~K}$, at $545 \mathrm{~K}$, at $620 \mathrm{~K}$ and at 680$850 \mathrm{~K}$ (107). The two lower temperature phases were similar, although not identical, to the surface oxide and chemisorbed oxygen species seen earlier. When CO was used to titrate chemisorbed oxygen both of the higher temperature features were left behind. Moreover, the highest temperature feature was found to grow and to shift to higher desorption temperatures as the ion energy and sputtering time were increased. The authors hypothesized that this feature must be associated with deep sub-surface oxygen and that the feature exhibiting a desorption peak at $\sim 620 \mathrm{~K}$ may be associated with oxygen released from the sub-surface by a reconstruction during the heating ramp. However, experiments revealed that the work function was only restored to the value for a clean $\mathrm{Au}(110)-(1 \times 2)$ surface when all the oxygen was removed. This data seems inconsistent with a sub-surface species and led the authors to suggest that the oxygen associated with high temperature desorption may be merely a strongly bound surface species. By LEED, the authors observed that the disorder induced by the adsorbed (and possibly absorbed) oxygen from $\mathrm{O}^{+}$bombardment could be reestablished by heating above $650 \mathrm{~K}$. With sufficient cooling, oxygen can be physisorbed on gold surfaces (106). Physisorbed oxygen on Au(110)- (1x2) could be observed to desorb in three peaks: a multilayer peak at $37 \mathrm{~K}$ and two peaks associated with the monolayer at 45 and $51 \mathrm{~K}$. The two monolayer peaks may be due to two different types of sites on the missing row reconstruction of $\mathrm{Au}(110)$ such as on top of the rows and down in the trenches. No physisorbed oxygen could be transferred to a chemisorbed state by simple heating of the surface.

Bär et al. have observed the formation of a surface oxide of gold on a FIM tip after exposure to 1 bar of $\mathrm{O}_{2}$ for 20 min at $450 \mathrm{~K}(108)$. The tip has many kink and step sites which may facilitate the dissociation process. Exposure to CO led to rapid removal of the oxide.
Like oxygen, hydrogen does not dissociate readily on Au single crystal surfaces. Sault et al. examined the adsorption of atomic hydrogen on $\mathrm{Au}(110)$ using the hot filament technique that was successful for atomic oxygen dosing (109). Hydrogen was observed to desorb in a single peak at 216 K. Stobinski found that while hydrogen and deuterium will not adsorb at all on sintered gold films, molecular chemisorption was observed on unsintered films at $78 \mathrm{~K}$ (110). Hydrogen and deuterium were both found to desorb between 120-125 K.

Kastanas and Koel examined the adsorption of $\mathrm{Cl}_{2}$ on $\mathrm{Au}(111)$ at $120 \mathrm{~K}$ and at $500 \mathrm{~K}(111)$. They found that for a $0.25 \mathrm{~L} \mathrm{Cl}_{2}$ an ordered $(3 \times 3) \mathrm{R} 30^{\circ}$ structure could be seen with LEED when the sample temperature was less than 230 K. Unlike the case for atomic oxygen the Au(111) spots could also be clearly seen (the reconstruction is lifted upon adsorption of chlorine). Chlorine was seen to desorb in two peaks: a molecular desorption peak at $640 \mathrm{~K}$ and an atomic desorption peak at $790 \mathrm{~K}$. No evidence of surface chloride formation or chlorine diffusion into the crystal bulk was observed.

Hydrocarbon adsorption on gold surfaces is of significant interest due to the number of partial hydrogenation and oxidation reactions that are potentially catalyzed by gold. Davis and Goodman compared propene adsorption on $\mathrm{Au}(111)$ and $\mathrm{Au}(100)$ (104) and found that the adsorption on the clean surfaces was remarkably similar. Propene desorbed in two peaks: a multilayer at $120 \mathrm{~K}$ and a monolayer peak at 140-145 K. No significant dissociation of propene was found to occur on either surface. Outka and Madix studied the adsorption of ethylene and acetylene on $\mathrm{Au}(110)$ (112). For acetylene adsorption, a single broad desorption peak between 125-200 K was observed with no signs of decomposition products. Ethylene TPD data looked nearly identical to acetylene, also with the absence of decomposition products.

Outka and Madix examined the adsorption of methanol (112), formic acid and formaldehyde (113) on Au(110) and observed reversible desorption without decomposition reactions for all of these molecules. Chesters and Somorjai also failed to observe dissociative adsorption of ethylene, cyclohexene, benzene and n-heptane on both $\mathrm{Au}(111)$ and the stepped surface $A u(6(111) \times(100))$ (96). In contrast, naphthalene was found to dissociate on both surfaces when exposed at room temperature. The resulting hydrocarbon fragments were very strongly bound indicating that the barrier to dissociation must be large.

Koel and co-workers have studied the adsorption properties of a variety of organic molecules on Au(111). Once again, acetone (114), benzene (115), cyclohexane (116), cyclohexene (116), nitrobenzene (117), and nitromethane (118) have all been shown to undergo reversible adsorption 
without decomposition on $\mathrm{Au}(111)$. Koel's group also used IRAS to study the adsorption geometries of these molecules. Wang et al concluded that nitromethane must be bonded through a single oxygen to the $\mathrm{Au}(111)$ surface (unlike $\mathrm{NO}_{2}$ which is doubly bonded) to the surface for all coverages considered (118). Nitrobenzene was found to exhibit a flat geometry for low coverages up to $1.0 \mathrm{ML}$, as evidenced by the lack of intensity of in-plane modes (117). At higher coverages where multilayers of condensed nitrobenzene are formed, the molecules began to exhibit a tilted geometry with regard to both the $Y$ and $Z$ axes. Acetone was found to bind through the oxygen atom and adopt a tilted geometry at low coverages (112). However, as coverage was increased to about $1 \mathrm{ML}$, the orientation of the molecules changed such that the $\mathrm{C}-\mathrm{O}$ bond was parallel to the surface and the molecular plane is perpendicular to the surface.

Christmann's group has examined the reversible adsorption of acetonitrile and benzonitrile (119). Neither molecule was found to exhibit ordered phases or to lift the surface reconstruction. Using a combination of work function measurements and HREELS, the authors concluded that acetonitrile bonds to the surface through its nitrogen atom with the C-C-N axis likely at some inclination to the surface. Benzonitrile was found to orient parallel to the surface as it bonded through three ring carbon atoms as well as an additional overlap between the $\mathrm{C}=\mathrm{N}$ and an underlying $\mathrm{Au}$ atom. Christmann and co-workers went on to study the reaction of these molecules with K-promoted Au surfaces as a model electrochemical system (120).

In addition to organic molecules, Koel's group has also looked at the adsorption of nitrogen oxides on $\mathrm{Au}(111) . \mathrm{NO}_{2}$ was found to adsorb reversibly exhibiting a monolayer desorption peak at $220 \mathrm{~K}$ and a multilayer desorption peak at $150 \mathrm{~K}$ (121). HREELS measurements indicated that the molecule was oriented in an upright position and bonded to the surface through its two oxygen atoms. In multilayer films significant dimerization of $\mathrm{NO}_{2}$ to form $\mathrm{N}_{2} \mathrm{O}_{4}$ was observed. Accompanying experiments showed that $\mathrm{NO}$ and $\mathrm{N}_{2} \mathrm{O}$ did not adsorb on Au(111) for temperatures above $95 \mathrm{~K}$. Building on the results from Koel's lab, ESR measurements showed that although the monolayer failed to produce an ESR spectra, isolated $\mathrm{NO}_{2}$ molecules could be detected in the multilayer on $\mathrm{Au}(111)$ (122). In the case of a multilayer, the monolayer acts to shield the condensed molecules above it from the spin exchange between the adsorbate spin and electrons in the conduction band of gold, which results in the loss of the spectra.

The poisoning effect of sulfur on catalysts is well known (123). As with other adsorbates, however, the interaction of sulfur with gold is somewhat unique and sulfur has even been found to act as a promoter in gold based catalysts (124). Rodriguez et al have studied the adsorption of sulfur on
$\mathrm{Au}(111)$ with photoemission spectroscopy over a wide range of coverages (125). Supporting DFT calculations confirmed earlier findings by Gottschlack and Hammer (126) that sulfur preferred the three-fold hollow site at low coverages and induced a reconstruction to $\mathrm{a} \sqrt{3} \times \sqrt{3} \mathrm{R} 30^{\circ}$ pattern as seen by LEED (127). As the coverage was increased beyond $\sim 0.35$ $\mathrm{ML}$, the LEED pattern vanished and a reduction in the strength of Au-S bonds, accompanied by the formation of $S_{2}$ species, was observed. At coverages above a monolayer, sulfur preferred the on-top site and sulfur oligomers began to form. Similar behavior was observed for sulfur adsorbed on polycrystalline gold.

Unlike many other metal surfaces, the adsorption of $\mathrm{H}_{2} \mathrm{~S}$ on $\mathrm{Au}(111)$ and $\mathrm{Au}(100)$ has been found to be completely reversible $(128,129)$. On the other hand, Jaffey and Madix have observed that about $2 \%$ of a monolayer of $\mathrm{H}_{2} \mathrm{~S}$ will decompose on $\mathrm{Au}(110)$ (130). Hydrogen was observed to desorb at $215 \mathrm{~K}$, indicating some decomposition had occurred and $\mathrm{a}_{2} \mathrm{~S}$ desorption at $270 \mathrm{~K}$ was attributed to a disproportionation reaction of $\mathrm{SH}$ groups on the surface. Repeating the previous experiment, Dwyer and co-workers, however, did not see decomposition of $\mathrm{H}_{2} \mathrm{~S}$ on $\mathrm{Au}(110)$ (131) and concluded that Jaffey and Madix's results were probably due to defects. In fact in their examination of freshly sputtered surfaces, Dwyer et al. observed very similar adsorption behavior to Madix and Jaffey.

As thiol molecules are frequently used to build selfassembled monolayers on gold surfaces, various researchers have untaken their study for sub-monolayer characterizations. Nuzzo et al. examined the adsorption of methanethiol and dimethyl disulfide on $\mathrm{Au}(111)$ and found while methanethiol was observed to experience reversible adsorption, dimethyl disulfide underwent decomposition on the surface (132). Liu et al (133) found similar chemisorption features for the adsorption of methanethiol on $\mathrm{Au}(111)$ as Nuzzo et al. However, decomposition products of dimethyl disulfide and methane were also observed to desorb from the surface at $470 \mathrm{~K}$ and $540 \mathrm{~K}$ respectively after exposure at $100 \mathrm{~K}$. The decomposition could be attributed to defects, although theoretical calculations by Sellers predicted that with the associative formation of hydrogen on the surface an essentially thermoneutral reaction resulted (134). More recent DFT calculations suggest deprotanation of methanethiol should occur on the terraces of $\mathrm{Au}(111)$ (135). Liu et al also found the desorption of thiophene to be influenced by the presence of defects as the main peak at $186 \mathrm{~K}$ had a high temperature shoulder extending out $330 \mathrm{~K}$ (133). The authors concluded that this latter feature must be associated with defects, although thiophene did not experience any decomposition. $\mathrm{SO}_{2}$ was found to interact only weakly with $\mathrm{Au}(111)$, desorbing in a monolayer peak at $142 \mathrm{~K}$, only $12 \mathrm{~K}$ above the multilayer feature. 


\section{Table 2}

Results from TPD experiments for various adsorbates on bulk gold surfaces

\begin{tabular}{|c|c|c|c|}
\hline Adsorbate & Gold Surface & Desorption Temperature & ref \\
\hline $\mathrm{CO}$ & polycrystalline & $170 \mathrm{~K}$ & 82 \\
\hline $\mathrm{CO}$ & $\mathrm{Au}(332)$ & $140 \mathrm{~K}, 185 \mathrm{~K}$ & 83 \\
\hline $\mathrm{CO}$ & $\mathrm{Au}(110)$ & multilayer $32 \mathrm{~K}$, bilayer $37 \mathrm{~K}$, physisorbed monolayer $55 \mathrm{~K}, 67 \mathrm{~K}$, chemisorbed $145 \mathrm{~K}$, defects $190-220 \mathrm{~K}$ & 61 \\
\hline Atomic $\mathrm{O}$ & polycrystalline & recombination to $\mathrm{O}_{2} @ 650 \mathrm{~K}$ & 95 \\
\hline Atomic $\mathrm{O}$ & $\mathrm{Au}(110)$ & recombination $\mathrm{O}_{2} @ 590 \mathrm{~K}$ & 87 \\
\hline $\begin{array}{l}\text { Atomic } \mathrm{O} \text { by } \\
\text { e-bombardment } \mathrm{O}_{2}\end{array}$ & $\mathrm{Au}(110)$ & $\mathrm{Au}_{2} \mathrm{O}_{3}$ decomposition to $\mathrm{O}_{2} 497 \mathrm{~K}$, chemisorbed $\mathrm{O}$ recombination to $\mathrm{O}_{2} 553 \mathrm{~K}$ & 105 \\
\hline Atomic O by O+ sputtering & $\mathrm{Au}(110)$ & surface oxide $415 \mathrm{~K}$, chemisorbed species $545 \mathrm{~K}$, phase transformation species $620 \mathrm{~K}$, bulk O (?) 850-680 K, & 107 \\
\hline $\mathrm{O}_{2}$ & $\mathrm{Au}(110)$ & multilayer $37 \mathrm{~K}$, monolayer $45 \mathrm{~K}, 51 \mathrm{~K}$ & 106 \\
\hline $\mathrm{O}_{3}$ & $\mathrm{Au}(111)$ & decomposition to $\mathrm{O}$ atoms- $550 \mathrm{~K}$ as $\mathrm{O}_{2}$ & 101 \\
\hline Atomic O & $\mathrm{Au}(100)$ & $\mathrm{O}_{2} 470 \mathrm{~K}$ & 104 \\
\hline $\mathrm{CO}_{2}$ & $\mathrm{Au}(110)$ & $105 \mathrm{~K}$ & 144 \\
\hline Atomic D & $\mathrm{Au}(110)$ & $216 \mathrm{~K}$ as $\mathrm{D}_{2}$ & 109 \\
\hline $\mathrm{D}_{2}$ & Polycrystalline & $125 \mathrm{~K}$ on defects & 110 \\
\hline $\mathrm{Cl}_{2}$ & $\mathrm{Au}(111)$ & $\mathrm{Cl}_{2} 640 \mathrm{~K}, \mathrm{Cl}$ atoms $790 \mathrm{~K}$ & 111 \\
\hline $\mathrm{H}_{2} \mathrm{O}$ & $\mathrm{Au}(110)$ & $160 \mathrm{~K}$ & 112 \\
\hline $\mathrm{C}_{2} \mathrm{H}_{4}$ & $\mathrm{Au}(110)$ & $125-200 \mathrm{~K}$ & 112 \\
\hline $\mathrm{C}_{2} \mathrm{H}_{2}$ & $\mathrm{Au}(110)$ & $125-200 \mathrm{~K}$ & 112 \\
\hline $\mathrm{CH}_{3} \mathrm{OH}$ & $\mathrm{Au}(110)$ & multilayer $175 \mathrm{~K}$, monolayer $200 \mathrm{~K}$ & 112 \\
\hline $\mathrm{CH}_{3} \mathrm{OH}$ & $\mathrm{Au}(111)$ & multilayer $140 \mathrm{~K}$, monolayer $160 \mathrm{~K}$ & 102 \\
\hline $\mathrm{CH}_{2} \mathrm{O}$ & $\mathrm{Au}(110)$ & $160 \mathrm{~K}$ & 113 \\
\hline $\mathrm{HCOOH}$ & $\mathrm{Au}(110)$ & multilayer $175 \mathrm{~K}$, monolayer $210 \mathrm{~K}$ & 113 \\
\hline $\mathrm{C}_{3} \mathrm{H}_{6} \mathrm{O}$ & $\mathrm{Au}(111)$ & multilayer $132 \mathrm{~K}$, bilayer $137 \mathrm{~K}$, monolayer $160 \mathrm{~K}$ & 114 \\
\hline $\mathrm{C}_{3} \mathrm{H}_{6}$ & $\mathrm{Au}(111)$ & multilayer $120 \mathrm{~K}$, monolayer $145 \mathrm{~K}$, & 104 \\
\hline $\mathrm{C}_{3} \mathrm{H}_{6}$ & $\mathrm{Au}(100)$ & multilayer $120 \mathrm{~K}$, monolayer $140 \mathrm{~K}$, & 104 \\
\hline $\mathrm{C}_{6} \mathrm{H}_{6}$ & $\mathrm{Au}(111)$ & multilayer $151 \mathrm{~K}$, bilayer $155 \mathrm{~K}$, monolayer $239 \mathrm{~K}$ & 115 \\
\hline $\mathrm{C}_{6} \mathrm{H}_{10}$ & $\mathrm{Au}(111)$ & multilayer $143 \mathrm{~K}$, monolayer $213 \mathrm{~K}$, & 116 \\
\hline $\mathrm{C}_{6} \mathrm{H}_{12}$ & $\mathrm{Au}(111)$ & multilayer143 K, monolayer $198 \mathrm{~K}$, & 116 \\
\hline $\mathrm{C}_{6} \mathrm{H}_{14}$ & $\mathrm{Au}(111)$ & multilayer $135 \mathrm{~K}$, second layer $144 \mathrm{~K}$, monolayer $210 \mathrm{~K}$ & 136 \\
\hline $\mathrm{C}_{10} \mathrm{H}_{8}$ & $\mathrm{Au}(111)$ & decomposition to hydrocarbon fragments & 96 \\
\hline $\mathrm{H}_{2} \mathrm{~S}$ & $\mathrm{Au}(111)$ & multilayer $103 \mathrm{~K}$, monolayer $165 \mathrm{~K}$ & 128 \\
\hline $\mathrm{H}_{2} \mathrm{~S}$ & $\mathrm{Au}(110)$ & multilayer $130 \mathrm{~K}$, monolayer $160-180 \mathrm{~K}$ & 131 \\
\hline $\mathrm{H}_{2} \mathrm{~S}$ & $\mathrm{Au}(110)$ & multilayer $130 \mathrm{~K}$, multiple states $155 \mathrm{~K}, 180-200 \mathrm{~K}, 220 \mathrm{~K}, \mathrm{H}_{2} 215 \mathrm{~K}, \mathrm{H}_{2} \mathrm{~S}$ from reaction $270 \mathrm{~K}$, S atoms & 130 \\
\hline $\mathrm{H}_{2} \mathrm{~S}$ & $\mathrm{Au}(100)$ & monolayer $107 \mathrm{~K}$ & 129 \\
\hline $\mathrm{CH}_{3} \mathrm{SH}$ & $\mathrm{Au}(111)$ & multilayer $135 \mathrm{~K}$, bilayer $190 \mathrm{~K}$, monolayer $228 \mathrm{~K}$ & 132 \\
\hline $\mathrm{CH}_{3} \mathrm{SH}$ & $\mathrm{Au}(111)$ & $120 \mathrm{~K}, 139 \mathrm{~K}, 176 \mathrm{~K}, 200 \mathrm{~K}$ dimethyl disulfide $470 \mathrm{~K}$, methane $540 \mathrm{~K}$ & 133 \\
\hline $\mathrm{C}_{4} \mathrm{H}_{4} \mathrm{~S}$ & $\mathrm{Au}(111)$ & monolayer $186 \mathrm{~K}$, defects $330 \mathrm{~K}$ & 133 \\
\hline $\mathrm{C}_{6} \mathrm{H}_{5} \mathrm{SH}$ & $\mathrm{Au}(110)$ & multilayer $190 \mathrm{~K}$, multiple states $200-350 \mathrm{~K}$, benzenethiol $630 \mathrm{~K}$, biphenyl $615 \mathrm{~K}$, dibenzothiophene $638 \mathrm{~K}$, S atoms & 139 \\
\hline $\mathrm{C}_{4} \mathrm{H}_{9} \mathrm{SH}$ & $\mathrm{Au}(100)$ & multilayer $138 \mathrm{~K}$, butene $500 \mathrm{~K}, \mathrm{~S}_{2} 940 \mathrm{~K}$ & 141 \\
\hline $\mathrm{C}_{2} \mathrm{H}_{5} \mathrm{SH}$ & $\mathrm{Au}(110)$ & $\begin{array}{l}\text { multilayer } 125 \mathrm{~K} \text {, 2nd layer } 200 \mathrm{~K} \text {, monolayer } 300 \mathrm{~K}, \mathrm{H}_{2} 195 \mathrm{~K}, \mathrm{H}_{2} 300 \mathrm{~K} \text {, ethane } 500 \mathrm{~K} \text {, ethylene } 500 \mathrm{~K} \text {, } \\
\text { ethanethiol } 525 \mathrm{~K} \text {, S atoms }\end{array}$ & 138 \\
\hline t-butyl thiolalcohol & $\mathrm{Au}(110)$ & multilayer $160 \mathrm{~K}$, mutiple states $180-340 \mathrm{~K}, \mathrm{H}_{2} 230 \mathrm{~K}, \mathrm{H}_{2} 330 \mathrm{~K}$, isobutene $470 \mathrm{~K}, \mathrm{H}_{2} \mathrm{~S} 470 \mathrm{~K}$, t-butyl thiol $470 \mathrm{~K}$, S atoms & 140 \\
\hline $\mathrm{C}_{2} \mathrm{H}_{6} \mathrm{~S}_{2}$ & $\mathrm{Au}(111)$ & decomposition & 132 \\
\hline $\mathrm{SO}_{2}$ & $\mathrm{Au}(111)$ & multilayer $130 \mathrm{~K}$, monolayer $142 \mathrm{~K}$, & 133 \\
\hline NO & $\mathrm{Au}(111)$ & $<95 \mathrm{~K}$ & 119 \\
\hline $\mathrm{N}_{2} \mathrm{O}$ & $\mathrm{Au}(111)$ & $<95 \mathrm{~K}$ & 119 \\
\hline $\mathrm{NO}_{2}$ & $\mathrm{Au}(111)$ & multilayer $150 \mathrm{~K}$, monolayer $220 \mathrm{~K}$ & 119 \\
\hline $\mathrm{C}_{6} \mathrm{H}_{5} \mathrm{NO}_{2}$ & $\mathrm{Au}(111)$ & multilayer $195 \mathrm{~K}$, bilayer $210 \mathrm{~K}$, monolayer $290 \mathrm{~K}$ & 117 \\
\hline $\mathrm{CH}_{3} \mathrm{NO}_{2}$ & $\mathrm{Au}(111)$ & multilayer $166 \mathrm{~K}$, monolayer $177 \mathrm{~K}$ & 118 \\
\hline $\mathrm{CH}_{3}-\mathrm{C}=\mathrm{N}$ & $\mathrm{Au}(100)$ & multilayer $150 \mathrm{~K}$, monolayer $175 \mathrm{~K}$ & 120 \\
\hline $\mathrm{C}_{6} \mathrm{H}_{5} \mathrm{CN}$ & $\mathrm{Au}(100)$ & multilayer $195 \mathrm{~K}$, shoulder $238 \mathrm{~K}$, monolayer $285 \mathrm{~K}$ & 120 \\
\hline $\mathrm{C}_{6} \mathrm{H}_{5} \mathrm{l}$ & $\mathrm{Au}(111)$ & multilayer $188 \mathrm{~K}$, bilayer $212 \mathrm{~K}$, monolayer $300 \mathrm{~K}$, biphenyl $400 \mathrm{~K}$ & 151 \\
\hline $\mathrm{CH}_{3} \mathrm{I}$ & $\mathrm{Au}(111)$ & multilayer $190 \mathrm{~K}$, monolayer $240 \mathrm{~K}$, decomposition on defects & 148 \\
\hline $\mathrm{CH}_{3} \mathrm{I}$ & $\mathrm{Au}(100)$ & multilayer $190 \mathrm{~K}$, monolayer $250 \mathrm{~K}, \mathrm{C}_{2} \mathrm{H}_{6} 360 \mathrm{~K}$ & 148 \\
\hline $\mathrm{C}_{2} \mathrm{H}_{5 \mathrm{l}}$ & $\mathrm{Au}(111)$ & $215 \mathrm{~K}$, decomposition products mostly $\mathrm{C}_{2} \mathrm{H}_{4}, \mathrm{C}_{2} \mathrm{H}_{6} 265 \mathrm{~K}$ & 149 \\
\hline $\mathrm{C}_{2} \mathrm{H}_{5} \mathrm{l}$ & $\mathrm{Au}(100)$ & $222 \mathrm{~K}$, decomposition products mostly $\mathrm{C}_{4} \mathrm{H}_{10} 260 \mathrm{~K}$ & 150 \\
\hline
\end{tabular}


For higher thiols evidence of surface decomposition has been seen by Nuzzo et al with AES on Au(111) (136). In their extensive study of the adsorption of sulfur containing hydrocarbons on Au(111), Lavrich et al demonstrated that the TPD of decanethiol could be substantially altered by annealing the thiol covered surface at $343 \mathrm{~K}$, and thereby inducing increased cleavage of S-H bonds (137). Madix and Jaffey observed significant decomposition of ethanethiol (138), benzenethiol (139) and tert-butyl thioalcohol (140) on the more open and thereby more reactive $A u(110)$ surface. A pattern in their reactivity emerged. In TDS spectra between 150 and $350 \mathrm{~K}, \mathrm{H}_{2}$ was observed to emerge from the surface as the $\mathrm{S}-\mathrm{H}$ bond was broken at the surface. Then as the surface temperature was increased above $400 \mathrm{~K}$, the appropriate thiolate group on the surface decomposed and a complex series of disproportionation reactions followed, yielding products that included the thiol itself as well as higher hydrocarbons and sulfided hydrocarbons. On sulfur covered surfaces, $\mathrm{H}_{2} \mathrm{~S}$ was formed in addition to hydrogen between 200 and $350 \mathrm{~K}$. The thiolate was found to be stabilized to higher temperatures on sulfur covered $\mathrm{Au}(110)$, and the reaction products generally had shifted distributions. Unlike the rather complicated chemistry of higher thiols on $\mathrm{Au}(110)$, butanethiol was seen to exhibit reversible adsorption on $\mathrm{Au}(100)$ (141). When butanethiol was adsorbed on a sulfur pre-covered surface, decomposition was observed, resulting in the desorption of 1-butene at $500 \mathrm{~K}$ and the presence of sulfur on the surface, which could either desorb at $940 \mathrm{~K}$ or disappear into the bulk upon annealing to high temperature. Based upon the small amount of sulfur necessary to induce decomposition, it was speculated that defects or impurities could be responsible for decomposition products seen on "clean" gold single crystal surfaces.

The observation by Gellman and co-workers that kinked surfaces possessed inherent chirality (142) has begun to be exploited by researchers for the adsorption of chiral thiols. Hammer and co-workers have calculated that the Senantiomer of 2-amino-3-dimethylphosphino-1-propanethiol adsorbs with $8.8 \mathrm{KJ} / \mathrm{mol}$ higher binding energy on $\mathrm{Au}(1711$ 9) as its R-enantiomer (143). Despite its preference for bridge bonding the thiolate group is predicted to bond to a single gold atom as the bonding of the phosphino group is optimized for the S-enantiomer. On the other hand, the Renantiomer cannot bond well to Au(17 11 9) due to a spatial mismatch between its functional groups and the active sites of the kinked surface.

In Table 1, the results for adsorption on bulk gold surfaces are summarized. Clearly very few adsorbates undergo dissociative adsorption or decomposition on bulk gold surfaces as one may expect. However, as we will discuss in the following section, bulk gold is capable of catalyzing some interesting reactions.

\subsection{Reactions on Au single crystals}

Under certain conditions it is possible to study at least partial reaction cycles and thereby gain insight into catalytic mechanisms using gold single crystals. CO oxidation at $300 \mathrm{~K}$ was observed by Parker and Koel when a Au(111) sample populated with atomic oxygen by exposure to ozone was subsequently exposed to CO (101). They saw a linear decrease in the reaction rate with oxygen coverage. Koel's

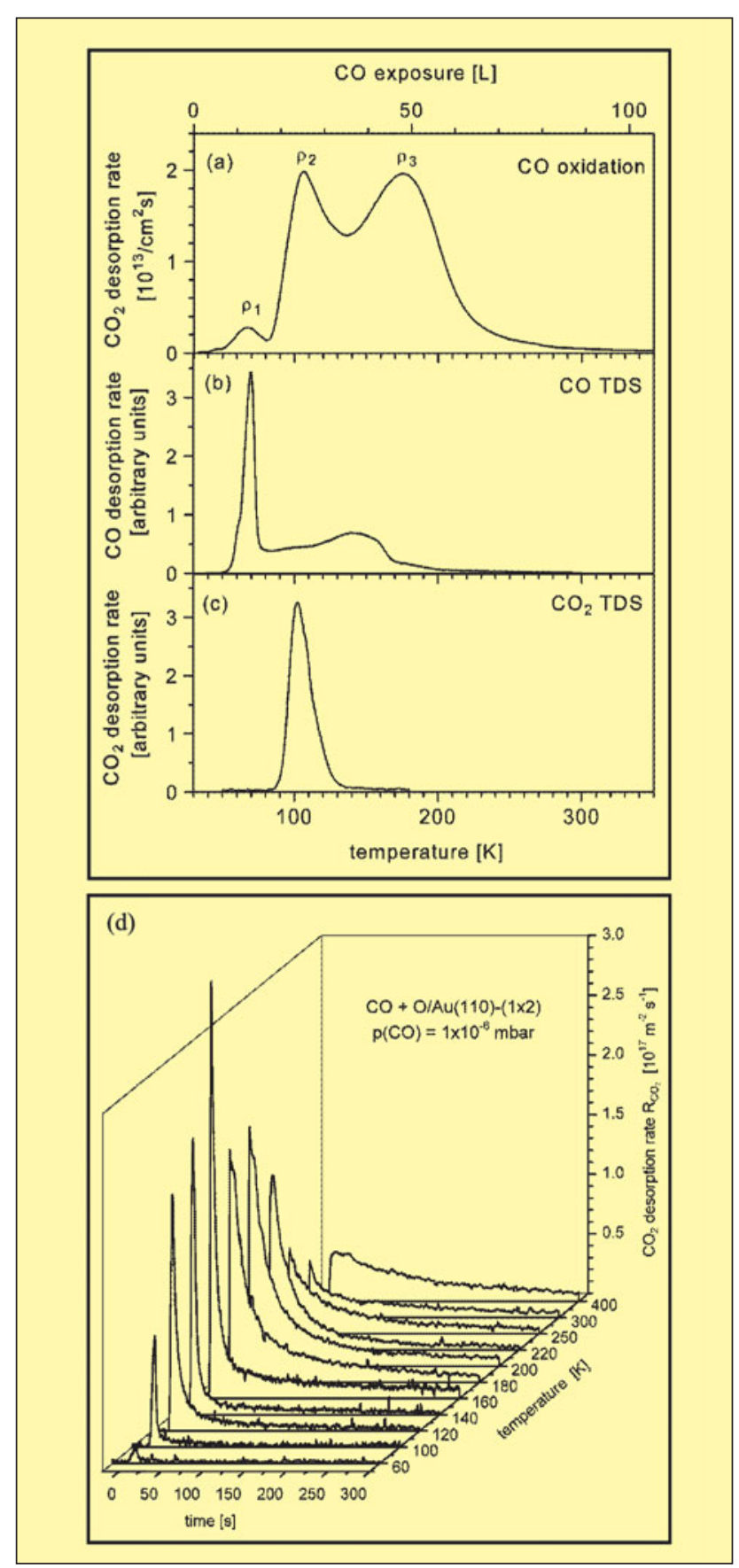

\section{Figure 6}

(a) $\mathrm{CO}_{2}$ production during $\mathrm{CO}$ exposure to O/Au(110)-(1x2). CO TPD spectrum (b) and $\mathrm{CO}_{2} / \mathrm{O} / \mathrm{Au}$ spectrum (c) are shown for comparison. (d) CO oxidation over O/Au(110)-(1x2) as a function of time for specified temperatures. $(144,145)$ 
group also observed a decrease in reaction rate with temperature between 250-375 K, which gave an apparent activation energy of $-2.5 \mathrm{kcal} / \mathrm{mol}$ (102). This value is consistent with several reported in the literature for real catalysts and the authors concluded that this effectively rules out an Eley-Rideal mechanism since a single step reaction cannot have a negative activation energy. Of course, in this experiment oxygen has already been provided to the surface and its dissociation may be the rate limiting step in the reaction process for real catalysts.

CO oxidation has been examined on Au(110) by Madix's and later by Christmann's group. Outka and Madix observed production of $\mathrm{CO}_{2}$ when a room temperature $\mathrm{Au}(110)$ single crystal covered with atomic oxygen was exposed to CO (87). Through the use of the $\mathrm{C}^{18} \mathrm{O}$ isotope, they were able to demonstrate that $\mathrm{CO}$ does not dissociate on the surface as $\mathrm{C}^{18} \mathrm{O}^{16} \mathrm{O}$ was the only reaction product observed. Additionally, no evidence of carbonate formation, which has been proposed by some researchers as a reaction intermediate, was found. Unlike Koel's group, Outka and Madix observed a positive apparent activation energy over a temperature range from 273-440 K. Based on their estimate for the desorption temperature of $\mathrm{CO}$, a real activation energy of $\sim 10 \mathrm{kcal} / \mathrm{mol}$ was calculated for an oxygen coverage of $0.25 \mathrm{ML}$. However, the authors also found that the reaction rate depended not only on the instantaneous oxygen coverage but also upon the initial oxygen coverage. They concluded that this phenomenon arose from the tendency of O-atoms to form islands at high coverages and that under these conditions CO oxidation occurs via an island eating mechanism.

Gottfried et al. examined $\mathrm{CO}_{2}$ production by TPD from $\mathrm{Au}(110)$ crystal covered by $1.3 \mathrm{ML}$ of atomic oxygen and then exposed to $\mathrm{CO}$ at $1 \times 10^{-6} \mathrm{mbar}$ as the sample was heated (144). As shown in Figure $6 \mathrm{a} \mathrm{CO}_{2}$ production was observed in three peaks: $67 \mathrm{~K}, 105 \mathrm{~K}$ and $175 \mathrm{~K}$. $\mathrm{CO}_{2}$ from the peak at 105 $\mathrm{K}$ was likely desorption limited as this coincides with the normal desorption temperature of $\mathrm{CO}_{2}$ from $\mathrm{Au}$ (110). Interestingly, the peak at $67 \mathrm{~K}$ indicated at least some $\mathrm{CO}_{2}$ produced was sufficiently excited by the reaction to overcome the desorption barrier. The feature at $175 \mathrm{~K}$ was attributed to the reaction limited production of $\mathrm{CO}_{2}$ which tails off sharply due to the low concentration of $\mathrm{CO}_{2}$ on the surface at elevated temperatures. In other experiments samples with an initial oxygen atom coverage of $0.45 \mathrm{ML}$ were exposed to $\mathrm{CO}$ at a various temperatures and the production of $\mathrm{CO}_{2}$ was observed over time as shown in Figure $6 \mathrm{~b}$ (145). Gottfried et al found that the reaction rate increased with temperature until about $170 \mathrm{~K}$ whereupon the production of $\mathrm{CO}_{2}$ began to decrease. At temperatures above $175 \mathrm{~K}$, the surface reaction was no longer the ratelimiting step as adsorption of $\mathrm{CO}$ controls $\mathrm{CO}_{2}$ production. It was also found that the presence of oxygen on the surface increased the desorption energy of $\mathrm{CO}$ such that the reaction rate decreased with time, not only from a depletion of oxygen, but also from a consequential decrease in the CO surface concentration (although the gas pressure was held constant). From this analysis, the authors determined an activation energy of $57 \mathrm{~kJ} / \mathrm{mol}$ (13.6 kcal/mol). In addition, annealing the surface after deposition of atomic oxygen was found to decrease the reaction rate, probably due to a loss of defects on the surface which aided in the adsorption of CO.

As mentioned in Section 2.2, Bär et al observed reaction of CO with a surface oxide on a gold FIM tip (108). When gas mixtures of $\mathrm{CO}$ and $\mathrm{O}_{2}$ were admitted to the chamber, a reaction front could be imaged on the tip. The presence of an electric field of 12-15 V/nm greatly enhanced the reaction.

Outka and Madix went on to explore the oxidation of other adsorbates as well. When acetylene was adsorbed on a partially oxygen-covered Au(110) surface, TPR experiments yielded reaction products of water and carbon dioxide, which were observed to desorb at $205 \mathrm{~K}$ and $525 \mathrm{~K}$ respectively (112). In a first step, adsorbed acetylene was dehydrogenated by oxygen to form water, which promptly desorbed. Later at much higher temperatures, the carbon left behind on the surface from dehydrogenation reacted with oxygen. In related work, no oxidation products were observed when ethylene was adsorbed on the oxygen covered surface.

The surface reaction of formic acid with oxygen atoms on $\mathrm{Au}(110)$ has also been studied in the Madix lab (113). Using a hot filament as before, the gold single crystal was populated with $0.25 \mathrm{MLO}$ atoms, and subsequently exposed to $0.1 \mathrm{ML}$ of formic acid at $100 \mathrm{~K}$. The sample was then heated and oxidation of formic acid was evidenced by desorption of water at $200 \mathrm{~K}$ and the subsequent desorption of $\mathrm{CO}_{2}, \mathrm{H}_{2} \mathrm{O}$ and formic acid at $340 \mathrm{~K}$. The evolution at $340 \mathrm{~K}$ of formic acid must have been reaction limited since it was so far above the desorption temperature of formic acid from the clean surface. Using isotopically labeled reactants, the authors deduced the following reactions whereby the formic acid reacts first with adsorbed oxygen to form water and a formate group. Later the formate groups could react to produce $\mathrm{CO}_{2}$ and formic acid or they could react with adsorbed oxygen to produce $\mathrm{CO}_{2}$ and water.

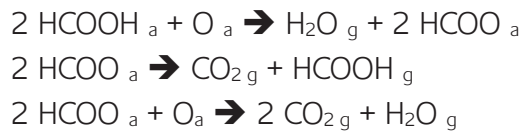

Using similar conditions, formaldehyde was seen to react on the oxygen covered $\mathrm{Au}(110)$ surface (113). Oxidation products of $\mathrm{H}_{2} \mathrm{O}$ at $215 \mathrm{~K}, \mathrm{H}_{2}$ at $230 \mathrm{~K}$, and $\mathrm{CO}_{2}, \mathrm{H}_{2} \mathrm{O}$ and $\mathrm{HCOOH}$ at $340 \mathrm{~K}$ were observed. Additionally $\mathrm{CH}_{2} \mathrm{O}$ was seen to desorb at $300 \mathrm{~K}$, most likely stabilized on the surface by adsorbed formate groups. The reaction scheme involved the reaction of formaldehyde with surface oxygen to form adsorbed formic acid and hydrogen. This formic acid could, 
in turn, react with surface oxygen in a similar way as above to produce formate groups which complete the scheme.

Outka and Madix also investigated the reaction of methanol with the oxygen covered surface (112). Following an exposure of methanol at $100 \mathrm{~K}$ to a $\mathrm{Au}(110)$ surface covered with $0.25 \mathrm{ML}$ oxygen, water was observed to desorb at $200 \mathrm{~K}$. Results using isotopes revealed that this water was produced from the hydroxyl abstraction by surface oxygen, leaving a methoxy group on the surface. Upon additional heating to $250 \mathrm{~K}$, some methanol desorbed as well as methyl formate and traces of hydrogen. Interestingly, the majority of the methanol observed to desorb at this temperature was not involved in the reaction but was stabilized on the surface by the presence of other species (oxygen, methoxy). Methoxy groups were proposed to react to form formaldehyde and some additional methanol, which desorbed. Unlike on $\mathrm{Cu}(111)$ (146) and $\mathrm{Ag}(111)$ (147), formaldehyde did not desorb as a product but reacted instead with methoxy groups to form methyl formate and hydrogen or with adsorbed oxygen to form formate groups. Further heating to $340 \mathrm{~K}$ produced $\mathrm{CO}_{2}$, resulting from formate decomposition or reaction of formate with oxygen (although no hydrogen was observed).

The adsorption of water on the oxygen covered surface of $\mathrm{Au}(110)$ was studied by Madix's group as well (112). Although no reaction occurs, the water was chemisorbed more strongly on the oxygen modified surface leading to a desorption peak at $215 \mathrm{~K}$ as opposed to $190 \mathrm{~K}$ on the clean surface. Koel and co-workers later found very similar results for the interaction of methanol, ethylene and water with atomic oxygen on the Au(111) surface as Outka and Madix's results on the $\mathrm{Au}(110)$ surface (102). This may indicate that these reactions studied are structure insensitive, upon adsorption of oxygen atoms.

Davis and Goodman have attempted to observe oxidation of propene on oxygen covered $\mathrm{Au}(111)$ and $\mathrm{Au}(100)$ in a similar manner (104). Davis and Goodman saw a broadening of the propene desorption peak as compared to the clean surface with an accompanying shoulder at $200 \mathrm{~K}$. Furthermore, significant amounts of the reaction products $\mathrm{H}_{2} \mathrm{O}$, CO and $\mathrm{CO}_{2}$ were observed to desorb. At an oxygen coverage of $0.5 \mathrm{ML}$, trace amounts of partial oxidation products such as acetone or propylene oxide were observed but not positively identified.

Other reactions can also be driven on Au single crystals. Paul and Bent examined the relationship between structure and reactivity of gold using $\mathrm{CH}_{3}$ l as a probe molecule. They found that on a well annealed $\mathrm{Au}(111)$ surface, $\mathrm{CH}_{3}$ l did not react (148). However, on sputtered surfaces a small amount of ethane formed at $270 \mathrm{~K}$, presumably due to partial dissociation of $\mathrm{CH}_{3} \mathrm{l}$ at defect sites and subsequent methyl coupling. On the $\mathrm{Au}(100)$ surface, even when well-annealed, considerable quantities of ethane are produced from $\mathrm{CH}_{3} \mathrm{l}$ adsorption. The coupling reaction on $\mathrm{Au}(100)$ did not occur until $330 \mathrm{~K}$, which Paul and Bent ascribed to a stronger $\mathrm{Au}-\mathrm{CH}_{3}$ bond on the more open $\mathrm{Au}(100)$ surface. They also observed that the methyl coupling reaction could be blocked by adsorption of trimethylphosphine (TMP). Heating the surface covered with $\mathrm{CH}_{3} \mathrm{l}$ and TMP, allowed for the observation of methyl radical desorption at $415 \mathrm{~K}$. Bent's group found that higher iodoalkanes were even more reactive than iodomethane on gold single crystals $(149,150)$. For $C_{2}-C_{4}$ iodoalkanes both disproportionation and coupling reactions were possible and occurred in the same temperature range, although their reaction channels were not competitive. Iodoethane was shown to produce $\mathrm{C}_{2} \mathrm{H}_{4}, \mathrm{C}_{2} \mathrm{H}_{6}$ and $\mathrm{C}_{4} \mathrm{H}_{10}$ between 260-270 K on both $\mathrm{Au}(111)$ (149) and $A u(100)$ (150). However, the yield of ethene and ethane was observed to saturate at a rather low coverage of $\mathrm{C}_{2} \mathrm{H}_{5}$ I whereas the yield of butane continued to increase until the monolayer was saturated. On the Au(100) surface the coupling reaction was strongly favored whereas disproportionation reactions were favored on $\mathrm{Au}(111)$. Higher iodoalkanes also showed an increased preference for disproportionation with increasing carbon number.

In a similar fashion, Syomin and Koel examined the formation of biphenyl produced as a result of coupling reactions from fragments of iodobenzene decomposition (151). lodobenzene was seen to partially decompose between 200 and $250 \mathrm{~K}$. Since no phenyl fragments were observed by IRAS it was concluded that their coupling reaction to biphenyl must be very fast at the surface temperatures involved. Biphenyl, whose formation on the surface was observable concomitant to $\mathrm{C}_{6} \mathrm{H}_{5}$ I decomposition by IRAS, was observed to desorb at $400 \mathrm{~K}$. Koel's group has successfully demonstrated electron induced decomposition (EID) to be highly selective in the scission of a single $\mathrm{C}-\mathrm{H}$ bond for many adsorbed hydrocarbons and thereby explored the coupling reactions of hydrocarbon groups on $\mathrm{Au}(111)(115,116,152)$. Electron bombardment (30 eV) of adsorbed benzene was found to yield phenyl groups as shown by IRAS $(115,152)$. Heating the surface resulted in evolution of benzene at about $200 \mathrm{~K}$, with longer exposure times yielding narrower benzene desorption peaks. Concurrently, phenyl groups were observed to undergo coupling reactions to form biphenyl which desorbed at 400 K. Through IRAS, Koel and co-workers established that the coupling reaction to biphenyl occurred at $165 \mathrm{~K}$. This result was somewhat surprising since similar reactions occur at much higher temperatures on copper and silver. Syomin and Koel further examined EID of cyclohexane on Au(111) $(116,152)$. Cyclohexane adopted a geometry parallel to the surface of gold. However, after electron bombardment, a 
cyclohexyl group was formed, which orients itself such that it was bound to the surface through a single Au-C bond. Heating the surface to $160 \mathrm{~K}$ caused the loss of a second hydrogen such that some cyclohexene was formed, which desorbed at the normal temperature of $215 \mathrm{~K}$. A second cyclohexene peak, accompanied by cyclohexane occurred at $273 \mathrm{~K}$ as a result of disproportionation reactions of the cyclohexyl groups. Another mass 82 peak $\left(\mathrm{C}_{6} \mathrm{H}_{10}{ }^{+}\right)$was observed at $324 \mathrm{~K}$, which was probably not due to cyclohexene, but was rather a cracking fragment of some higher unidentified hydrocarbon.

Bartram and Koel reported that $\mathrm{NO}$ will react with adsorbed $\mathrm{NO}_{2}$ on $\mathrm{Au}(111)$ at $100 \mathrm{~K}$ producing $\mathrm{N}_{2} \mathrm{O}_{3}$, as seen by HREELS (119). However, heating the surface to $170 \mathrm{~K}$ reversed the reaction and desorption of NO was observed. Wang and Koel also examined the interaction of $\mathrm{NO}_{2}$ with $\mathrm{H}_{2} \mathrm{O}$ (153). After exposing an ice film on $\mathrm{Au}(111)$ to $\mathrm{NO}_{2}$ at 86 $\mathrm{K}$, thermal desorption spectra revealed a complicated reaction network producing, most importantly, atomic oxygen on the surface.

\subsection{Bimetallic systems involving Au}

The general opinion that gold is an inert material has led researchers intuitively to utilize Au to selectively block different adsorption sites and therefore change the reaction routes. Au deposited on Si(111)-(7x7) surface will block the oxidation of the neighboring silicon, even at very low coverages (154). This seems to imply that gold not only blocks adsorption of underlying atoms but also changes the chemical nature of the silicon atoms it neighbors. Gold has also been used to decorate steps of ruthenium, effectively shutting off the dissociation of nitrogen on a gold modified Ru(0001) single crystal (155). Dahl et al demonstrated that deposition of less than 0.01 ML Au on the Ru(0001) surface reduced the sticking coefficient of $\mathrm{N}_{2}$ by over seven orders of magnitude at $500 \mathrm{~K}$.

Gold doesn't always act to merely lower the surface reactivity. Surface science studies of bimetallic systems involving gold have revealed some very interesting chemistry. In their investigation of thin Pd films on $\mathrm{Au}(111)$, Smith and co-workers found that gold modified the CO adsorption capability of Pd $(156,157)$. Photoemission and HREELS data indicated that CO adsorption on the Pd monolayer was considerably weaker than on bulk Pd due to a decrease of electron density near the Fermi level. In Sellidj and Koel's examination of thin Pd films grown on $\mathrm{Au}(111)$ (158), the binding energy of CO was found to be slightly reduced at very low Pd coverages. However, when the surface was completely covered with a Pd layer, the CO adsorption was very similar to $\mathrm{Pd}(111)$. The authors note that interpretation of the data is complicated by alloying processes that occur concomitant to the temperature
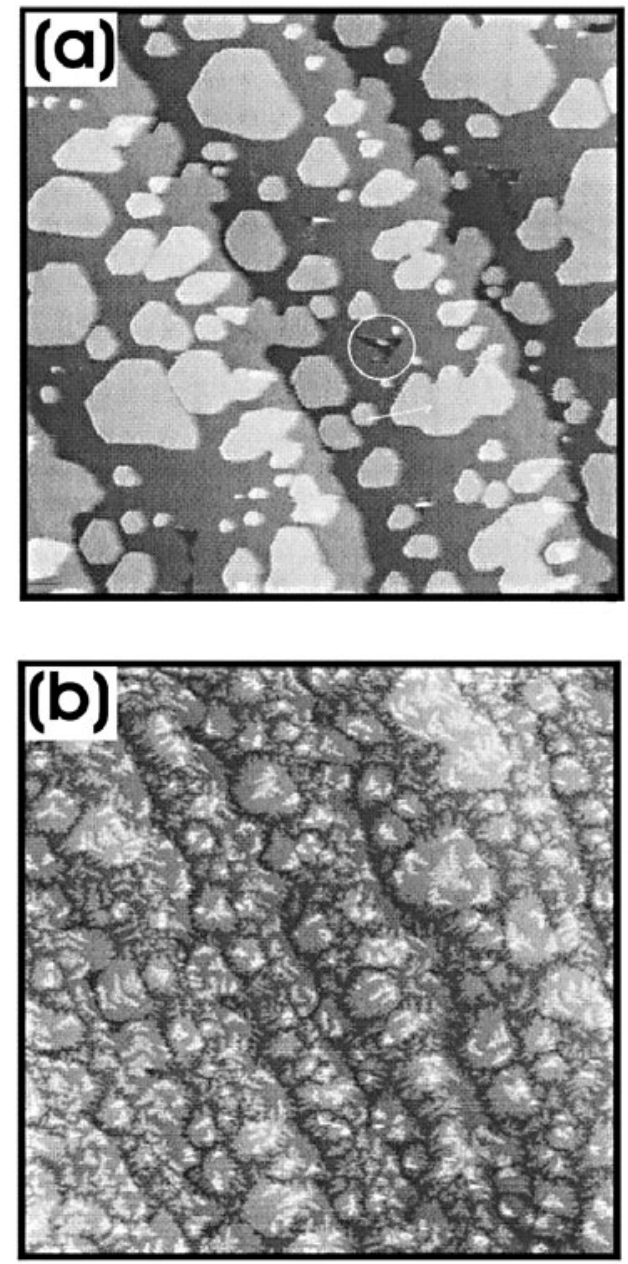

\section{Figure 7}

(a) STM image of a Pd(111) surface covered by $1.5 \mathrm{ML}$ Au at $320 \mathrm{~K}$. (b) STM image of Au/Pd(111) surface after additional deposition of 0.25 ML Au at $110 \mathrm{~K}$. (Size $\left.335 \times 335 \mathrm{~nm}^{2}\right)$. (162)

programmed desorption. Pedersen et al also observed a decrease in $\mathrm{CO}$ binding strength for thin platinum films on $\mathrm{Au}(111)$ (159). The authors explain that Pt atoms with mostly gold neighbors lack a contribution in binding energy that they would normally experience on the Pt(111) surface, which therefore results in a lowering of the CO desorption temperature. However, as coverage of platinum was increased, an increase in the binding energy of CO on a Ptmodified $\mathrm{Au}(111)$ surface was observed, showing a maximum CO desorption temperature at a Pt coverage of 1.3 ML about $40 \mathrm{~K}$ above that of Pt(111). The results were correlated with DFT calculations that indicated that the increase in CO binding energy was due to a shift in the valence states of the Pt atoms in the Pt-Au surface alloy. Pedersen et al believe that the $d$ band shift originated from the lattice expansion by the Pt islands, which were found by STM to have a lattice constant of $2.86 \AA$.

In a reverse experiment, Schmitz et al. examined the adsorption of CO on very thin gold films grown on Pd(110) 
(160). In much the same way that palladium was less reactive when in contact with gold, TPD experiments revealed that gold is more reactive when in contact with palladium. In Ruff et al's characterization of Au deposits on $\operatorname{Pd}(111)(161,162)$ by STM, Au was found to completely cover the $\mathrm{Pd}(111)$ surface at coverages as low as $1.5 \mathrm{ML}$. Using EELS, the authors demonstrated that while CO did not adsorb in any significant quantity on such films, further gold deposition resulted in the creation of a unique site with a CO stretching frequency of $2120 \mathrm{~cm}^{-1}$. This adsorption site was believed to be associated with gold steps or Au island edges created by second deposition step. Figure $7 \mathrm{a}$ and $\mathrm{b}$ show STM images of gold deposited at $100 \mathrm{~K}$ and $300 \mathrm{~K}$ demonstrating low and high CO adsorption capacity respectively.

The properties of thin gold films or alloys have been investigated for other systems as well. Sachtler et al observed that monolayer gold films on Pt(100) were about 4 times more active than the bare Pt surface for the dehydrogenation of cyclohexene to benzene (163). Okada et al observed that epitaxially grown thin gold films on $\operatorname{lr}(111)$ could dissociate hydrogen and deuterium, in contrast with results for $\mathrm{Au}$ single crystals (164).

Besenbacher et al found that addition of gold to a $\mathrm{Ni}(111)$ single crystal resulted in a decrease in methane dissociation on the surface (165). The initial sticking probability of methane on a Ni surface with just $0.25 \mathrm{ML}$ of gold fell to $\sim 10 \%$ of the sticking value of the clean $\mathrm{Ni}(111)$ surface (166). DFT calculations revealed that the $\mathrm{CH}_{4}$ dissociation barrier on a $\mathrm{Ni}$ atom increased by $16 \mathrm{~kJ} / \mathrm{mol}$ when a single Au neighbor was present (167). Two neighboring gold atoms, resulted in a $38 \mathrm{~kJ} / \mathrm{mol}$ increase (the barrier to dissociation on a $\mathrm{Ni}(111)$ atom was calculated to be $\sim 100 \mathrm{~kJ} / \mathrm{mol}$ ). The calculations show that since the methane molecule interacts with the $d$ states of nickel in the dissociation process, the loss of reactivity can be explained by a lowering of $d$-band center when $\mathrm{Ni}$ is in contact with $\mathrm{Au}$. Furthermore, the gold modified surface also had a reduced tendency to bind carbon and form graphite (which leads ultimately to catalyst deactivation). Besenbacher et al then went on to synthesize a high surface area $\mathrm{Au} / \mathrm{Ni}$ on $\mathrm{MgAl}_{2} \mathrm{O}_{4}$ catalyst (16 wt \% Ni, 0.3 wt $\% \mathrm{Au}$ ) that demonstrated a remarkable improvement in durability over a pure Ni catalyst for steam reforming of methane as the reduced carbon uptake compensates for the loss of methane reactivity (165).

Baddeley et al found that Au deposited on Pd(111) made for a much better acetylene cyclization to benzene catalyst (168). The ideal surface was found to contain 18\% gold. The presence of gold served to lower the benzene desorption temperature, which is believed to be the ratelimiting step.

\section{Chemistry of gold clusters}

Although we have focused our discussion to this point upon extended surfaces, any discussion of gold chemistry seems incomplete without some mention of gas phase experiments with very small gold clusters. In real catalytic systems, gold is typically supported on metal oxides such as $\mathrm{TiO}_{2}, \mathrm{Fe}_{2} \mathrm{O}_{3}, \mathrm{Al}_{2} \mathrm{O}_{3}$ or $\mathrm{SiO}_{2}$. However, as we will discuss later, the nature of support can dramatically influence the reactivity of gold deposits. Therefore, in order to look at intrinsic properties of gold small particles, many groups have initiated studies of unsupported gold clusters.

One of the pioneering papers on gold chemistry that effectively foreshadowed the discovery of gold's catalytic oxidation ability, was Huber et al's surprising finding that a single gold atom could catalyze the oxidation of $\mathrm{CO}$ at temperatures as low as $10 \mathrm{~K}$ (169). When co-condensing Au atoms in a matrix of a $\mathrm{CO}: \mathrm{O}_{2}$ 1:1 mixture, a new species was formed, a carbonylgold(II) peroxyformate, which upon heating to $30-40 \mathrm{~K}$ decomposed, producing two $\mathrm{CO}_{2}$ molecules.

Since that time, other researchers have employed gas phase methods either as flow reactors or in ion traps to examine the reactivity of gold clusters. Cox et al. examined adsorption of $\mathrm{D}_{2}, \mathrm{O}_{2}$ and $\mathrm{CH}_{4}$ on various sized gold clusters $(170,171)$. The authors found that for cationic gold clusters of less than 16 atoms, deuterium readily adsorbed. However, for larger clusters no reaction occurred. For neutral gold clusters only $\mathrm{Au}_{3}$ and $\mathrm{Au}_{7}$ clusters were reactive with $\mathrm{D}_{2}$. Anionic clusters were found to be completely unreactive with deuterium. Anionic clusters with an even number of atoms were found to react with oxygen, although clusters with odd numbers of atoms did not adsorb oxygen. For cationic clusters only $\mathrm{Au}_{10}$ adsorbed oxygen. Methane was found to react with cationic clusters in a similar way as hydrogen with saturation conditions occurring for the same number of $D_{2}$ molecules as $\mathrm{CH}_{4}$ molecules on any given cluster size. When using mixtures of $\mathrm{CH}_{4}$ and $\mathrm{D}_{2}$, no higher mass products than the saturation number for any given cluster were seen, thus indicating that deuterium and methane compete for adsorption. Cox et al drew an analogy between electron affinity and the reactivity of their clusters. Clusters with the highest electron affinities had the highest reactivity to deuterium and methane while clusters with the lowest electron affinities had the highest reactivity to oxygen.

Lee and Ervin later confirmed Cox et al's finding for the reaction of anionic gold clusters between one and seven atoms (172) with $\mathrm{D}_{2}, \mathrm{O}_{2}$ and $\mathrm{CH}_{4}$. In addition, Lee and Ervin discovered that such clusters were unreactive with $\mathrm{NH}_{3}$ and $\mathrm{N}_{2}$, but did react with $\mathrm{CO}$. They found that $\mathrm{Au}_{n}{ }^{-}(\mathrm{n}=1-4)$ were considerably less reactive than larger clusters. The formation of dicarbonyl species for $n=4,5$ and tricarbonyl species for 
$n=6,7$ were seen as well. The variations were determined to result from the particulars of the bonding. For example, CO binds to the clusters via $\sigma$ donation into the cluster LUMO and $\pi$ back-bonding from the $d$ orbital electrons of the cluster. Both of these are weak interactions that are not considered to be highly sensitive to the structure or size of the particle. On the other hand, oxygen is believed to bond through its unpaired electron in the $\pi^{*}$ antibonding orbital with the HOMO of the cluster. Logically, this interaction is favored when the particle possesses an odd number of electrons such that an unpaired electron lies in HOMO, available for bonding.

Lian and co-workers found that neutral gold dimers would react with $\mathrm{CO}, \mathrm{NH}_{3}$ and $\mathrm{C}_{2} \mathrm{H}_{4}$, but not with $\mathrm{O}_{2}, \mathrm{~N}_{2} \mathrm{O}, \mathrm{N}_{2}$ or $\mathrm{CH}_{4}$ (173). Most unusually, hydrogen was seen to react with gold dimers at a slow rate, independent of hydrogen pressure. As $\mathrm{Ag}$ and $\mathrm{Cu}$ dimers were not seen to react with hydrogen, the authors concluded that the relativistic nature of gold is likely responsible for the increased reactivity of $\mathrm{Au}_{2}$, as the s-orbital is contracted and stabilized, leading to a shorter and more stable Au-H bond.

Using UPS, Smalley's group observed even-odd oscillations of up to $1.0 \mathrm{eV}$ in electron affinities, with the largest oscillations seen for gold clusters with less than 20 atoms (174). This could be explained by the relativistic effects in gold, whereby strong mixing is observed between the $s$ and $d$ orbitals. For clusters of 20 atoms or less, $d$-band features are not observed. For clusters with upwards of 70 atoms the valence region was found to be essentially indistinguishable from the bulk. Smalley and co-workers observed very local maximums in the HOMO-LUMO gaps (and minimums in electron affinity) for gold clusters with closed shell sizes of 8 , 20, 34 and 58 atoms. Employing this idea of shell closing to reactivity studies, Smalley and co-workers examined the adsorption of CO on cationic gold particles (175). As expected, maximums in the abundance were found for $\mathrm{Au}_{7}{ }^{+} \mathrm{CO}$ and $\mathrm{Au}_{19}{ }^{+} \mathrm{CO}$.

Li et al recently examined 20 atom gold clusters with anion photoelectron spectroscopy (176) and found that these structures possessed a remarkably high HOMO-LUMO gap, even greater than $\mathrm{C}_{60}$ (Buckey Balls), which should be an indicator of exceptional stability and perhaps chemical inertness. Accompanying DFT calculations revealed a tetrahedral pyramid to be the most stable structure, although an $\mathrm{Au}_{20}$ planar structure was revealed to be nearly as favorable. The authors speculated that the tetrahedral clusters may have unusual catalytic properties due to their high surface area and large number of highly uncoordinated atoms.

Employing a gas flow reactor system, Salisbury et al also found that adsorption of oxygen occurred readily on anionic gold clusters with an even number of atoms (with exceptions at $\mathrm{n}=16$ and $\mathrm{n}=22$ ) whereas clusters with an odd number of atoms did not react (177). In no case did the authors observe a secondary adsorption of oxygen. As discussed above, this result could be explained by a mechanism in which oxygen adsorbs molecularly as a one-electron acceptor. As the gold cluster would then have fully paired electrons in its $\mathrm{HOMO}$, it is inactive to secondary adsorption. A similar examination by Stolcic et al of the interaction of anionic gold clusters of up to 27 atoms with oxygen confirmed the finding of Salisbury et al. and additionally showed that no particles with over 20 atoms adsorb oxygen (178). For these larger clusters, it was speculated that their higher electron affinity prevented oxygen adsorption, as the adsorption process is conceived to take place through a charge transfer from the gold to the anti-bonding orbital of oxygen. When the particle is so strongly electron withdrawing, no bonding can take place. Kim et al also found that the adsorption of oxygen on anionic gold clusters was strongly tied to the electron affinity (179). However, the valence band of Aun changed completely upon adsorption of oxygen and the peak from the HOMO of the anionic cluster (particularly for $\mathrm{n}=8-18$ ) disappeared, suggesting charge transfer from the HOMO to oxygen. At the same time, no correlation between the geometric structure of gold and its adsorption activity was found.

Wallace and Whetten studied the adsorption of $\mathrm{CO}$ on anionic gold clusters and found that certain preferred products such as $\mathrm{Au}_{5} \mathrm{CO}, \mathrm{Au}_{11} \mathrm{CO}, \mathrm{Au}_{15} \mathrm{CO}$ and $\mathrm{Au}_{15}(\mathrm{CO})_{2}$ were created $(180,181)$. These results also imply that CO adsorption can be structurally sensitive which has not been previously widely recognized. Interestingly, even at very high $\mathrm{CO}$ exposures, complexes with high numbers of $\mathrm{CO}$ molecules were not formed. This seems consistent with $\mathrm{CO}$ adsorption on single crystal surfaces which saturate at very low coverages. Hagen et al have recently examined the adsorption of $\mathrm{CO}$ on anionic monomers, dimers and trimers of gold in a ion trap experiment (182). Although CO was not found to react on $\mathrm{Au}^{\prime}$, carbonyl complexes with one or two $\mathrm{CO}$ molecules attached were observed for $\mathrm{Au}_{2}{ }^{-}$and $\mathrm{Au}_{3}{ }^{-}$. The formation of $\mathrm{Au}_{3}(\mathrm{CO})_{2}{ }^{-}$is strongly favored, which fits well with the idea of shell closing $\left(\mathrm{Au}_{3}(\mathrm{CO})_{2}{ }^{-}\right.$has an electron count of 8). However, the authors warn that simple examination of the $s$ orbital electron counts is insufficient as $s$ - $d$ hybridization in gold due to relativistic effects must not be ignored.

Taking the next logical step in an examination of $\mathrm{CO}$ oxidation, Wöste and co-workers recently observed that $\mathrm{CO}$ and $\mathrm{O}_{2}$ could be co-adsorbed on anionic two and three atom gold clusters, although no adsorption occurred on the monomer ion (183). The reaction of these clusters with oxygen was found to be at least an order of magnitude faster than with $\mathrm{CO}$ and therefore no gold carbonyls were observed. After formation of $\mathrm{Au}_{3}(\mathrm{CO}) \mathrm{O}_{2}$ it was possible to adsorb a second oxygen molecule to form $\mathrm{Au}_{3}(\mathrm{CO})\left(\mathrm{O}_{2}\right)_{2}$ indicating a 
cooperative adsorption process in which the adsorbates are not competing for bonding sites. Wallace and Whetten confirmed that this result is also true for larger clusters, i.e. the presence of one adsorbate facilitates the adsorption of the other (184). However, Wallace and Whetten produced exciting evidence for $\mathrm{CO}_{2}$ production as well. By exposing a group of $\mathrm{Au}_{6}{ }^{-}$clusters to $\mathrm{CO}$ in the upstream portion of the reactor, a distribution of $\mathrm{Au}_{6}(\mathrm{CO})_{\mathrm{M}}$ was created. Then this group of clusters was exposed to oxygen downstream and the products revealed the presence of $\mathrm{Au}_{6}(\mathrm{CO})_{\mathrm{M}-1} \mathrm{O}^{-}$, indicating (at least indirectly) an oxidation of a $\mathrm{CO}$ molecule and loss of $\mathrm{CO}_{2}$. An analysis of the reaction rate reveals that the reaction rate may be over 100 times that of a real gold catalyst. However, these small clusters undergo only a single reaction cycle at most so any comparison with real or model catalysts should be tempered with the knowledge that the measurement of reaction rates is usually over thousands of reaction cycles.

In a new study by Socaciu et al using an octopole ion trap (185), as opposed to the flow reactor system employed by Whetten and Wallace (184), the authors report the first ever measurement of turn-over-frequencies of anionic gold dimers for $\mathrm{CO}$ oxidation. Socaciu et al observed three products upon exposure of $\mathrm{CO}$ and $\mathrm{O}_{2}$ to $\mathrm{Au}_{2}{ }^{-}$species in the trap: $\mathrm{Au}_{2}^{-}, \mathrm{Au}_{2} \mathrm{O}_{2}^{-}$and $\mathrm{Au}(\mathrm{CO}) \mathrm{O}_{2}^{-}$. A simple mechanism was proposed by the authors to explain their findings:

$$
\begin{gathered}
\mathrm{Au}_{2}{ }^{-}+\mathrm{O}_{2} \rightarrow \mathrm{Au}_{2} \mathrm{O}_{2}{ }^{-} \\
\mathrm{Au}_{2} \mathrm{O}_{2}{ }^{-}+\mathrm{CO} \rightarrow \mathrm{Au}_{2}(\mathrm{CO}) \mathrm{O}_{2}{ }^{-} \\
\mathrm{Au}_{2}(\mathrm{CO}) \mathrm{O}_{2}+\mathrm{CO} \rightarrow \mathrm{Au}_{2}+2 \mathrm{CO}_{2}
\end{gathered}
$$

Interestingly, when the trap was cooled sufficiently ( $100 \mathrm{~K}$ ) and a high pressure of $\mathrm{CO}$ was employed $\mathrm{CO}$ could adsorb first on $\mathrm{Au}_{2}{ }^{-}$leading to the formation of a different type of $\mathrm{Au}_{2}(\mathrm{CO}) \mathrm{O}_{2}{ }^{-}$intermediate which does not react with $\mathrm{CO}$ to make $\mathrm{CO}_{2}$. Unfortunately, no direct measurement of $\mathrm{CO}_{2}$ production was possible. DFT calculations indicated a carbonate type species, as seen in Figure $8 \mathrm{a}$, formed by the Eley Rideal insertion of $\mathrm{CO}$ into the O-O bond of $\mathrm{Au}_{2} \mathrm{O}_{2}{ }^{-}$was the most stable type of intermediate. However, the loss of CO by the reverse reaction shown above would be unfavorable leading the authors to conclude that a gold-peroxyformate type species, as depicted in 8b, which is formed by an Eley Rideal reaction between $\mathrm{CO}$ and $\mathrm{Au}_{2} \mathrm{O}_{2}{ }_{2}$ without an activation barrier as seen by Ozin and coworkers (169) must also be present.

Balteanu et al have used Fourier Transform Ion Cyclotron Resonance Mass Spectrometry (186), to study the coadsorption of oxygen and CO. In agreement with the preceding results oxygen was observed to react much more readily with clusters that possessed preadsorbed $\mathrm{CO}$, which suggested cooperative adsorption. Furthermore, as in Wallace and Whetten's work (184) the reaction of CO was found to be highly size selective with both bare anionic

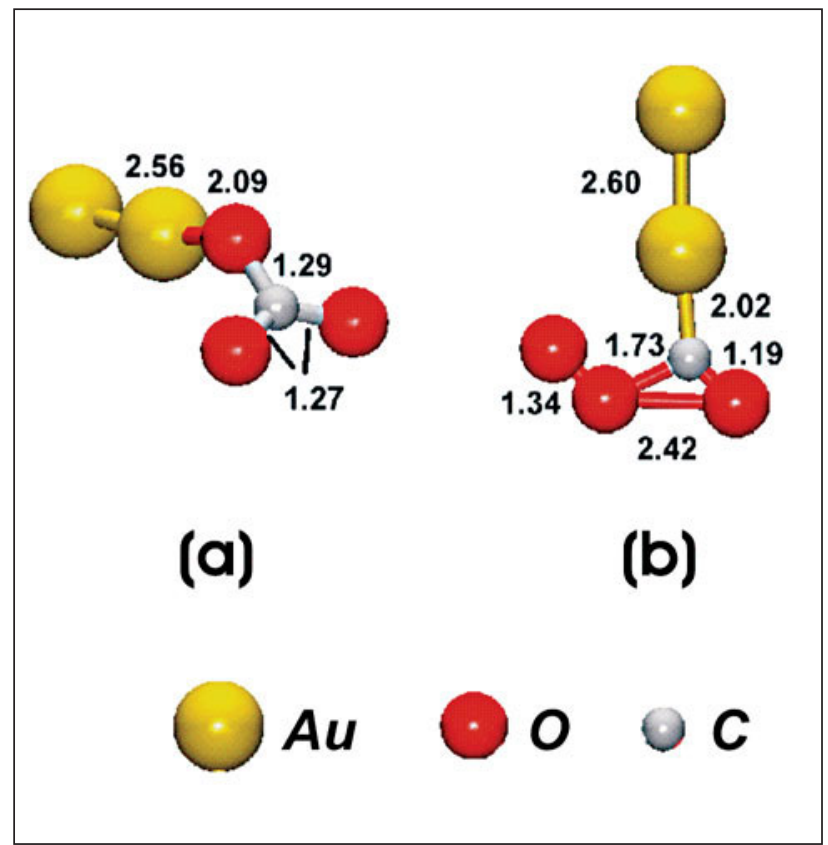

\section{Figure 8}

Two forms of $\mathrm{Au}_{2} \mathrm{CO}_{3}$ relevant to $\mathrm{CO}_{2}$ production. a) digold carbonate and b) digold-peroxyformate with calculated bond lengths indicated. (185)

clusters and with the complexes of $\mathrm{Au}_{N}(\mathrm{CO})_{\mathrm{M}}$. Although the products were efficiently co-adsorbed, no conclusive evidence for $\mathrm{CO}_{2}$ production was observed.

The effect of moisture on the adsorption properties of gold clusters has been examined by Wallace et al (187). A humid source allowed for the creation of $\mathrm{AuNOH}_{\mathrm{N}} \mathrm{Ol}^{-}$clusters which were then reacted with oxygen. The hydroxylated clusters exhibited the opposite trend from the bare clusters as even- $\mathrm{N}$ clusters were unreactive and odd- $\mathrm{N}$ clusters adsorbed oxygen readily. The authors propose that the high electron affinity of the $\mathrm{OH}$ group leads to an electron transfer such that bare odd-N Aun clusters which had no unpaired electrons possessed an unpaired electron available for bonding after adsorption of $\mathrm{OH}$.

Cationic gold clusters from 1-12 atoms have been recently examined by Sugawara et al for their ability to interact with $\mathrm{H}_{2} \mathrm{~S}$ (188). Again, definite patterns with regard to the ionization potential (or electron affinity) emerged. $\mathrm{Au}^{+}$and $\mathrm{Au}_{3}{ }^{+}$were completely unreactive and, as a general rule, even numbered particles had a higher activity. Multiple reaction products were possible as more than one $\mathrm{H}_{2} \mathrm{~S}$ molecule could adsorb and in some cases release of hydrogen was seen. Sugawara et al also tested the reaction of these cationic clusters with $\mathrm{H}_{2}$. They observed hydrogenated reaction products for clusters of up to 10 atoms. In some cases multiple hydrogen adsorption was observed. For example, for five atom cationic gold clusters up to eight hydrogen atoms could be adsorbed. 


\section{Theory of gold surface chemistry}

Theorists have also taken up the challenge to explain the unique chemistry of gold. In their examination of $\mathrm{H}_{2}$ dissociation on Au(111), Hammer and Nørskov found that two factors were critical to the activation barrier for reaction (and the strength of adsorbate-metal interaction as well) (189). First, gold, like all coinage metals, has a filled $d$-band, which gives rise to repulsion when an adsorbate such as hydrogen comes in contact with the surface due to filling of the antibonding orbital. Second, the degree of orbital overlap is very high for gold as compared to silver or copper resulting in a large orthogonalization energy cost and thus makes the adsorption character of gold unique.

Mavrikakis et al (190) have expanded this work to examine $\mathrm{CO}, \mathrm{O}$ atom and $\mathrm{O}_{2}$ adsorption and reaction on gold surfaces. DFT calculations indicate that CO prefers to chemisorb on the stepped surface $\mathrm{Au}(211)$ as compared to regular (111) terraces. Calculations for $\mathrm{CO}$ on $\mathrm{Au}(211)$ indicate a bridge bonded adsorption state with a similar binding energy as what has been found experimentally by Ruggerio and Hollins on $\mathrm{Au}(332)$ (83). O atoms were also found to prefer the stepped surface. $\mathrm{O}_{2}$ was found to adsorb only at steps, but the oxygen is only weakly bound, implying desorption may occur before dissociation. Whatever the nature of oxygen on the surface may be, the authors concluded that steps are an important parameter in the reactivity of small gold particles and showed that the reactivity of small particles as seen by Haruta and others $(191,192)$ could be correlated with their expected step densities as seen in Fig 9. The data correspond even better with results from experiments when one assumes that gold atoms in the first layer are unreactive (as shown by the squares in Fig 9). Nørskov and co-workers found that 0 adsorption on the monolayer islands is weaker than on thicker islands and CO should not adsorb at all on the monolayer islands. However, they acknowledge that if these islands are supported by a substrate which induces strain, the strained gold lattice should have an enhanced CO adsorption capability. Strain was also found to favorably effect $\mathrm{O}_{2}$ dissociation.

Mills et al have examined the adsorption of $\mathrm{O}_{2}$ on $\mathrm{Au}(111)$ and on ad-islands on $\mathrm{Au}(111)$ (193). They found that surface roughness, i.e. the presence of highly uncoordinated atoms was necessary for oxygen adsorption. In accordance with previous gas phase cluster experiments, odd numbers of electrons served to increase the binding of oxygen, although adding electrons to the flat $\mathrm{Au}(111)$ surface had no effect on the result. Calculations showed that the reactivity of rough surfaces and clusters results from a highly localized $\mathrm{HOMO}$ that allows for facile charge transfer into the $\pi^{*}$ orbital of $\mathrm{O}_{2}$. On smooth or extended surfaces the $\mathrm{HOMO}$ is delocalized such that the surface cannot bond with $\mathrm{O}_{2}$.

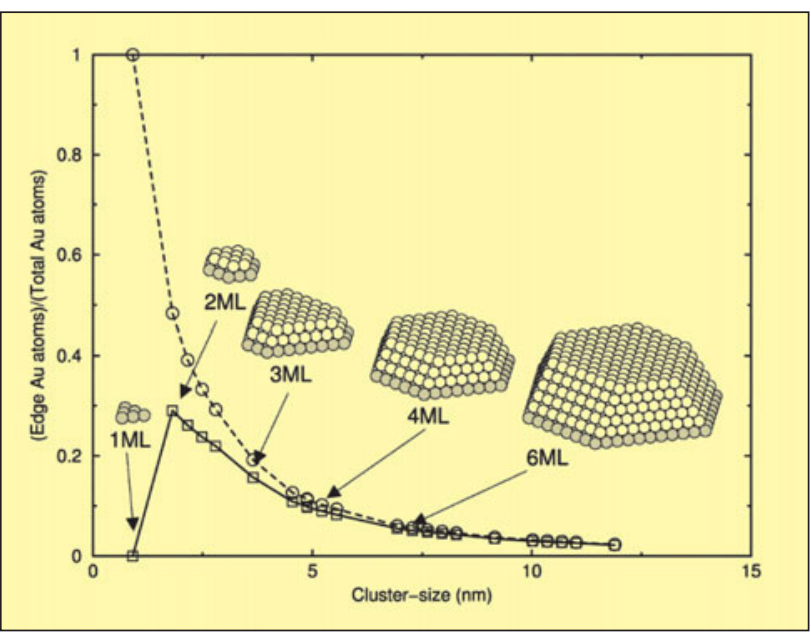

Figure 9

Calculated step density as a function of particle size. (190)

In addition to the presence of steps, $\mathrm{Xu}$ and Mavrikakis have demonstrated that strained surfaces should also greatly increase the binding energy of oxygen to gold as well as lower the barrier to dissociation (194). A 10\% strained surface of $\mathrm{Au}(211)$ was found to exhibit a binding energy of about double that of its unstrained counterpart $(0.26 \mathrm{eV}$ to 0.15 $\mathrm{eV}$ ) and the reaction barrier was almost halved (from $1.12 \mathrm{eV}$ to $0.63 \mathrm{eV}$ ).

In Strømsnes et al's examination of oxygen adsorption on $\mathrm{Au}(100)$ clusters of various sizes, calculations predicted adsorption energies of $\sim 60 \mathrm{kcal} / \mathrm{mol}$ for clusters with 17 or more atoms (195), in line with the experimental value estimated by Koel et al. (56 kcal/mol) (101). However, DFT simulations also predicted a decrease in the adsorption energy with decreasing cluster size, which is counter to observations on supported particles (196). Accompanying calculations for $\mathrm{H}$-atom adsorption predict an opposite trend: decreasing adsorption energy with increasing cluster size.

Liu et al has also examined $\mathrm{CO}$ and $\mathrm{O}_{2}$ adsorption and reaction on gold surfaces (197). Surprisingly, they found that steps are more reactive than kinks for the adsorption of oxygen atoms, in spite of their higher coordination number. Steps were also found to possess the lowest barrier (0.93 eV) to dissociation for $\mathrm{O}_{2}$, even lower than small Au clusters of 12 atoms. Using their calculated values, they estimated a dissociation probability of $\sim 10^{-21}$ for steps on $\mathrm{Au}(211)$ based on a room temperature ideal gas, indicating that for any unsupported gold surface essentially no dissociation of oxygen occurs. Like Mavrikakis et al (186), Liu et al found that CO had a strong preference for adsorption at steps, although adsorption was also strong at adatoms and kinks. Liu et al then examined the CO oxidation reaction for the $\mathrm{Au}(221)$ surface. In their reaction scheme involving atomic oxygen, $\mathrm{CO}$ and an $\mathrm{O}$ atom are both adsorbed in a bridge-bonded fashion along a step edge as seen in Figure 10a. In the 


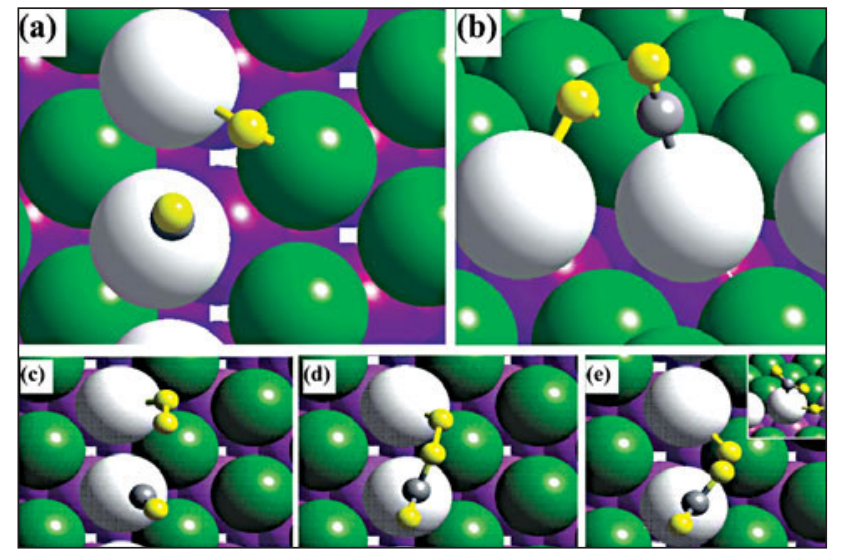

Figure 10

(a) Top and (b) side views of the transition state for $\mathrm{CO}_{2}$ production from the reaction of $\mathrm{CO}$ with atomic oxygen on $\mathrm{Au}(221)$. (c-e) show the progression of reaction between $\mathrm{CO}$ and molecular oxygen on Au(221). See text for details. (197)

transition state CO moves to an on-top site on the step edge and oxygen moves to a bridge site between a step atom and a terrace atom. The reaction to $\mathrm{CO}_{2}$ was found to have an activation barrier of $0.25 \mathrm{eV}$, much lower than that of Pt(111) for example $(0.80 \mathrm{eV})$. In a reaction with molecular oxygen, the proposed reaction scheme is essentially an Eley-Rideal type mechanism where $\mathrm{O}_{2}$ in the gas phase approaches CO adsorbed at a step edge and reacts leaving an oxygen atom behind on the surface as seen in Figure 10b. The activation barrier for this reaction was found to be $0.59 \mathrm{eV}$. However, when a second CO molecule is present, the barrier is lowered to $0.46 \mathrm{eV}$, showing that CO coverage could be critical to the reaction's success. It should be mentioned that the transition state proposed by the authors is not a symmetrical carbonate species, which has been proposed in other reaction schemes. Instead they find that in the transition O-O-CO complex, the two $\mathrm{O}$ atoms of $\mathrm{O}_{2}$ are chemically different from the oxygen of $\mathrm{CO}$.

Although such a reaction with molecular oxygen could take place on other metals, their ability to dissociate oxygen precludes it. However, since oxygen is not dissociated by gold, a reaction pathway involving molecular oxygen is conceivable. The authors concluded the adsorption of $\mathrm{CO}$ and possible dissociation of oxygen are not related to particle size but rather to step densities on the particles. They point out that for the real catalyst the role of the support may be to facilitate the adsorption of oxygen. In this case the reaction would proceed along the molecular oxygen pathway as described above. However, the reaction would have a LangmuirHinshelwood mechanism as opposed to Eley-Rideal.

Other researchers have examined the shape of free gold particles and the possible implications for adsorption behavior. Using a combination of ion mobility measurements and DFT calculations, Furche et al found that gold anionic clusters of up to at least 11 atoms preferred a planar arrangement (198). The authors believe that the relativistic nature of gold is responsible for gold's late transition from two-dimensional structures to three-dimensional particles compared to other metals. Häkkinen et al confirmed this result with DFT calculations of their own that showed a planar or raft-like structure is the optimal geometrical arrangement for anionic gold clusters up to 13 atoms $(33,199)$.

For neutral clusters, Wang et al found that small clusters (up to 6 atoms) prefer a planar arrangement, while for clusters between 7 and 9 atoms 3-dimensional geometries were preferred (200). For clusters between 10 and 14 atoms a flat cage structure possesses the lowest energy configuration. For clusters with greater than 15 atoms compact nearly spherical configurations were the most stable structures. In a similar way to experiments examining ionization energies, the HOMO-LUMO gap was found to have oscillations for even and odd numbers of atoms. The sensitivity of the density of states to cluster structure was also demonstrated by Wang et al in their comparison of the DOS for icosahedral, cubooctahedral and amorphous 13 atom clusters. Häkkinen and Landman had previously calculated that neutral gold clusters of up to 7 atoms preferred a two-dimensional arrangement (201). Grönbeck and Andreoni have also confirmed a planar arrangement for both anionic and neutral gold clusters from 2 to 5 atoms (202).

An interesting question arises about the electronic nature of these two dimensional particles. Zhao et al carried out calculations on planar particles to determine their polarizability (203). In the lateral direction the polarizabilities were found to be large (like a metal). However, the clusters displayed non-metallic character in the normal direction. For a very large two-dimensional Au cluster, the polarizability in the normal direction is essentially equal to a semiconductor like Si. Calculated densities of states revealed discrete behavior for 4 atom clusters and, as cluster size was increased to 20 atoms, a slow transition to a band-like DOS was observed.

Theoretical predictions for the structure of larger (between 30-1000 atoms) gold clusters have also been performed. To date, there is some debate over the lowest energy arrangements of such clusters, as proposals for disordered structures $(204,205)$ and various types of faceted particles $(176,206-210)$ have both been put forward. As pointed out by Marks (201), the determination of the correct equilibrium structures of small particles is complicated by changes that can occur during observation due to the influence of the HREM (212) and due to the influence of the underlying support. Furthermore, there may be only small differences in energy between structures allowing for facile transformation (205). 
Of particular interest is the transition between fcc structures which predominant for very large clusters, and decahedral or icosahedral particles, whose formation is predicted to be favored for very small particles. Whereas Cleveland et al. predicted that gold should transition from fcc structures to decahedral particles for sizes below 200 atoms and to icosahedral particles at sizes below 100 atoms (210), Uppenbrink and Wales found that the transition from fcc structures to icosahedral particles should occur at 550 atoms for gold (209). As we will discuss in the following section, the details of the structure may have strong implications for its reactivity.

Computational methods have also examined adsorption on small gold clusters. DFT calculations performed by Okumura et al show that $\mathrm{AuO}_{2}{ }^{-}$is considerably more stable than $\mathrm{AuO}_{2}$ (213). Calculations of $\mathrm{Au}_{13}$ clusters showed an optimized arrangement for $\mathrm{O}_{2}$ adsorption in which the surface atoms had some partial negative charge. Based on these findings, the authors suggest that negatively charged surface atoms are active sites for oxygen adsorption for supported gold particles. In similar calculations, Mills et al. report that oxygen adsorption is strongly preferred on even numbered anionic gold clusters as opposed to odd numbered or neutral clusters as has been observed in gas phase experiments (214). Bonding is conceived to take place by means of a charge transfer from gold to the anti-bonding $\pi^{*}$ orbital of $\mathrm{O}_{2}$. Wells et al. calculated that while oxygen bonding was weak for nine and eleven atom anionic gold clusters, it adsorbed readily on $\mathrm{Au}_{10}{ }^{-}$(215). Wells et al reported a different geometry for the bonding arrangement as they favor an edge on attachment to the HOMO of the cluster as opposed to the bent-end-on configuration favored by Okumura et al and Mills et al Yoon et al and Franceschetti et al have also examined the reaction of $\mathrm{O}_{2}$ with anionic gold clusters $(216,217)$. Calculations showed that for $n>3$, oxygen would prefer to be adsorbed dissociatively. However, the energy barrier for dissociation computed by Yoon et al was still quite high (2.33 eV) for $\mathrm{Au}_{6} \mathrm{O}_{2}{ }^{-}$, which is the most favorable case (216).

Metiu and co-workers advise some caution with regard to application of DFT to questions involving the adsorption of oxygen on gold (218). DFT calculations using both B3LYP and PW91 functionals were found to lead to prediction of significant overbinding of oxygen to gold clusters as compared to experimental findings and cluster methods.

Wu et al considered CO adsorption in free gold clusters between one and six atoms and calculated that CO preferred to adsorb in a single-coordinated fashion regardless of cluster size or the cluster's charge state (219). The charge state did, however, influence the strength of the interaction as cationic clusters bind CO more strongly. The nco vibrational frequencies were predicted to decrease from $2228 \mathrm{~cm}^{-1}$ for

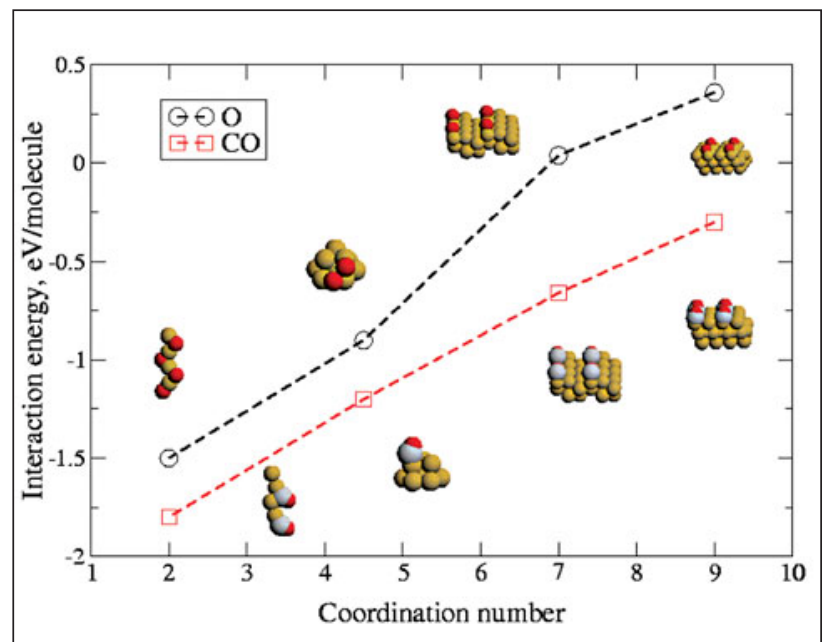

\section{Figure 11}

Interaction energy in eV per molecule for $\mathrm{CO}$ and $\mathrm{O}_{2}$ adsorption versus coordination number for gold atoms in various geometries. (222)

$\mathrm{n}=1$ to $2166 \mathrm{~cm}^{-1}$ for $\mathrm{n}=6$, but were always above the gas phase value $\left(2143 \mathrm{~cm}^{-1}\right)$. The clusters themselves were found to prefer a flat or raft-like orientation, both with and without cO adsorbed.

Häkkinen and Landman have also calculated the oxidation of CO on an anionic gold dimer (220). They determined that the most favorable arrangement should be a carbonate complex $\mathrm{Au}_{2} \mathrm{CO}_{3}{ }^{-}$. The carbonate can then react with a second $\mathrm{CO}$ to produce two $\mathrm{CO}_{2}$ molecules. In alternate pathway the carbonate can simply decompose, releasing $\mathrm{CO}_{2}$ and leaving $\mathrm{Au}_{2} \mathrm{O}^{-}$behind, which is then available for reaction with $\mathrm{CO}$.

In the study of a neutral ten atom gold cluster, Lopez and Nørskov found that $\mathrm{O}_{2}$ dissociation was very facile (221) which had not been previously seen on $\mathrm{Au}(111)$ or $\mathrm{Au}(211)$ $(190,197)$. The increased reactivity of the ten atom cluster is attributed to a higher lying $d$ state as compared with atoms on single crystal surfaces. However, the geometry of the cluster is highly critical to its ability to adsorb oxygen. In their model a two layer raft-like structure was chosen with three atoms lying atop a seven atom layer. This is in contrast with the $\mathrm{Au}(111)$ type geometry that was examined previously by Mavrikakis et al for a twelve atom cluster (190). The authors found that both $\mathrm{O}_{2}$ and $\mathrm{O}$ atoms adsorbed on $\mathrm{Au}_{10}$ could react with $\mathrm{CO}$ with reaction barriers less than $0.4 \mathrm{eV}$. Nørskov's group studied the effect of geometry in more detail, calculating the variation of the strength of adsorption with the coordination number of gold (222) as shown in Figure 11.

Examination of gold nanowires with a coordination of two, revealed that $\mathrm{CO}$ experienced very strong adsorption on the order of $2 \mathrm{eV}$, which is approximately equivalent to $\mathrm{CO}$ binding to more reactive surfaces such as nickel or platinum. Perhaps even more surprising, oxygen was found to 
dissociate in a highly exothermic manner and insert itself into gold chains forming an alternating string of gold and oxygen atoms. The authors suggest that the gold nanowires that have been previously cited for their long bond distances (223) may in fact be oxygen-gold chains. Recently, it has been suggested that hydrogen could also be responsible for the stabilization of large Au-Au bond distances (224).

Metiu and co-workers have interpreted their results of calculations examining the adsorption of propene on gold clusters and surfaces in a slightly different way (225). Rather than a direct relationship between coordination number and adsorption strength, Chrétien et al developed a set of semiempirical rules regarding the position of the LUMO of a particular gold cluster, as the bonding of propene was conceived to occur through donation of electron density from its HOMO into the particles LUMO. Therefore, propene adsorbed most favorably at the point at which the lowest lying LUMO extends the furthest into vacuum. Furthermore, the lower the LUMO, the higher the binding energy. Only in cases where the LUMO density would be equivalent would the coordination number become a critical factor. An adatom on a gold surface becomes a naturally favorable adsorption site as its LUMO extends into the vacuum far more than the underlying terrace, which by nature has a highly delocalized LUMO. In addition, it was found that an adatom of gold on a gold surface should be more reactive to propene than an adatom of gold above a vacancy on a $\mathrm{TiO}_{2}$ surface as the former case is electron depleted compared to the latter and thereby accepts an electron more easily. Of course for an electron-accepting adsorbate, like $\mathrm{O}_{2}$, this would be the opposite and adsorption to Au above the defective oxide would be favored.

\section{Gold model catalysts \\ 5.1 Growth and Nucleation of Gold}

Of course, in real heterogeneous catalysts, gold is usually dispersed on a metal oxide support. Therefore, questions also arise about the role of the support and the origin of any differences in catalytic activity between gold catalysts on various supports $(191,226)$. Therefore, in addition to research on single crystal surfaces as described in Section 2, many researchers have begun to examine gold particles deposited on either metal oxide single crystals or thin films, whereby the oxide is a model for the catalytic support. Surface science studies of planar model catalysts have been demonstrated to provide a bridge of the materials gap between "real" catalysts and metal single crystals (227-235).

Of these, gold deposited on $\mathrm{TiO}_{2}$ rutile(110) remains the most widely studied system involving gold to date. Cosandey and Madey have written an excellent review of the $\mathrm{Au} / \mathrm{TiO}_{2}$ system (236) and we will attempt to highlight some of this work as well as discuss results for other model catalysts. The (110) face of rutile has been examined extensively with STM as reviewed by Diebold (237). The (1x1) surface consists of alternating rows of titanium and oxygen atoms with half of the titanium atoms covered by so-called bridging oxygen. This leads to the creation of both 6-fold coordinated $\mathrm{Ti}$ (as in the bulk) and fivefold coordinated Ti, as well as threefold coordinated oxygen (as in the bulk) and two fold coordinated oxygen (bridging oxygen). Many researchers believe that due to their undercoordinated bonding, the bridging oxygens can be easily removed to form point defects or color centers, which strongly influence the support's chemistry. These defects can take on different forms depending upon whether oxygen is removed as $\mathrm{O}^{-}, \mathrm{O}^{+}$, or $\mathrm{O}^{\circ}$. Pacchioni has recently predicted that the removal of an oxygen atom on $\mathrm{TiO}_{2}$ results in a redistribution of charge over the Ti ions surrounding the vacancy such that the binding energy of the five-fold coordinated $\mathrm{Ti}$ atoms experience negative binding energy shift and the six-coordinated (of which there are now fewer) undergo a positive binding energy shift (238). This is in contrast to a surface like $\mathrm{MgO}$ where the charge left behind is strongly localized (239). A strongly reduced $\mathrm{TiO}_{2}(110)$ surface may undergo reconstruction to the $(1 \times 2)$ structure, although the exact details are still an area of active research (237). Due to the strong relationship between nucleation and defect density, surface preparation and characterization are very important to chemistry observed.

Studies by Zhang et al (240), Lai et al (241), Guo et al (242), Santra et al (243), Spiridis et al (244) and Parker et al (245) all confirm that gold experiences a two dimensional growth mode or quasi-2-D growth for very low coverages (< $0.15 \mathrm{ML}$ ) and then subsequently changes to a 3-D growth mode. STM images appear to show growth of gold at low coverages above Ti rows (241), but no atomically resolved images of both the particle and support simultaneously have been achieved such that one can make an unambiguous assignment. Santra et al observed that the nucleation density of particles at the step edges saturated much faster than on the terraces and that the average height of particles nucleated at step edges grew much faster than particles on terraces (243). Spiridis et al observed similarly strong preferential nucleation at step edges (244). As mentioned above there is substantial evidence that $\mathrm{F}$ centers $(3+$ defects on the $\mathrm{TiO}_{2}$ surface) act as nucleation sites for gold particles and may help stabilize the particles. Lai et al found that STM images of the $\mathrm{TiO}_{2}(110)$ surface covered with $0.1 \mathrm{ML}$ of gold appeared free of defects, thus implying that the small amount of gold on the surface must have nucleated at the defects and thereby obscures them from view (241). Using low energy ion scattering, Parker et al showed that ion bombardment of a $\mathrm{TiO}_{2}(110)$ surface to produce defects led to a higher nucleation density for gold deposited on the 

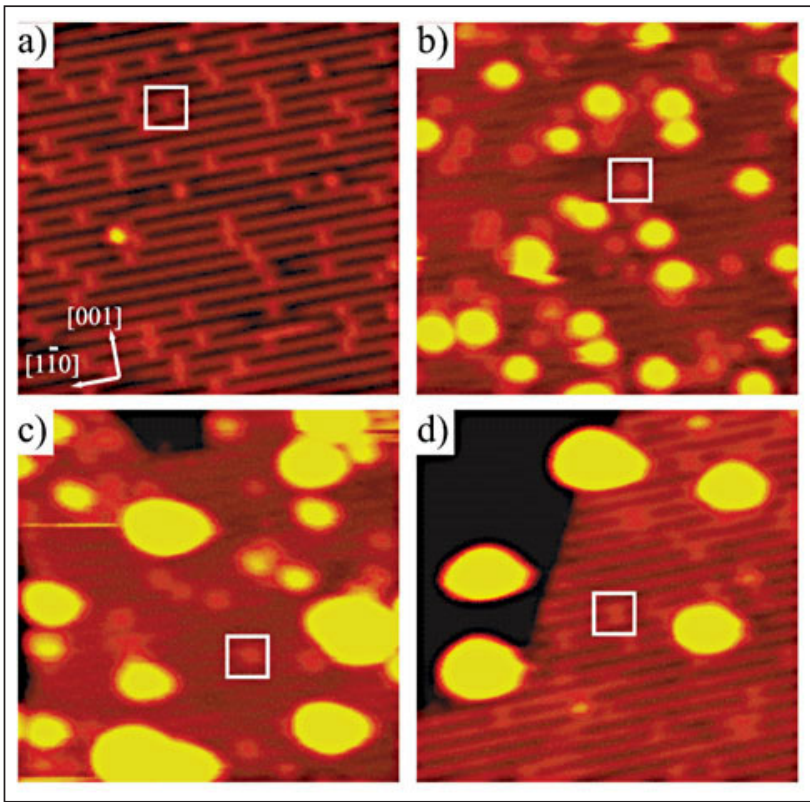

Figure 12

STM images of the (a) clean $\mathrm{TiO}_{2}(110)$ surface and with $0.04 \mathrm{ML} \mathrm{Au}$ deposited at (b) 130, (c) 210, and (d) $300 \mathrm{~K}$, showing the decrease in the number of exposed bridging oxygen vacancies (marked by squares) with increasing gold deposition temperature. (Image size $15 \times 15 \mathrm{~nm}^{2}$ ). (246]

defective surface (245). On the clean as prepared surface, the critical gold coverage for the transition from two dimensional growth to 3-D growth was found to be $0.09 \mathrm{ML}$ whereas on the sputtered surface the critical coverage was found to be $0.22 \mathrm{ML}$. Parker et al also saw that the temperature of the $\mathrm{TiO}_{2}(110)$ crystal during gold deposition can greatly affect the nucleation density. The critical coverage for gold was found to be $0.19 \mathrm{ML}$ for gold deposited at $160 \mathrm{~K}$ as opposed to $0.09 \mathrm{ML}$ for gold deposited at $300 \mathrm{~K}$, demonstrating that the growth mode is more three dimensional at higher temperatures. Indeed, Zhang et al reported that gold deposited at a sample temperature of $475 \mathrm{~K}$ will experience three-dimensional growth from the beginning on $\mathrm{TiO}_{2}$ (110) (240).

Recently, Wahlström et al has probed the relationship between gold particle nucleation and oxygen vacancies on a $\mathrm{TiO}_{2}(110)$ single crystal in detail (246). STM measurements showed the number of vacancies was drastically reduced upon the deposition of gold, even though gold occupied only a small fraction of the sample surface. As shown in Figure 12, when depositing gold at higher temperatures the reduction in vacancies was even larger, leading the authors to conclude that the particles trapped multiple oxygen vacancies beneath them. DFT calculations revealed that gold atoms prefer to bind to oxygen vacancies by more than 0.45 $\mathrm{eV}$ as compared to any site on the ideal titania surface. A single vacancy was found to be capable of binding up to 3 atoms. A transition between 2-D particles and 3-D particles was shown to be present for particles with less than 3 atoms per vacancy and more than three atoms per vacancy, respectively. Unlike calculations performed by Sanchez et al (247) for the binding of gold clusters on MgO, no significant charge transfer was seen.

The growth and nucleation behavior of gold on various supports has been modeled by numerous researchers. To date there has been a lack of agreement on the most stable adsorption sites for gold on oxide surfaces. For titania, various researchers have established that the on-top site above a fivefold coordinated Ti (248), the on-top site above a bridging oxygen $(249,250)$, bridge bonded between two bridging oxygen atoms (249), between a bridging oxygen and a 5 fold coordinated $\mathrm{Ti}$ on the basal plane (250), a four-fold hollow over 5 fold coordinated $\mathrm{Ti}$ and both a bridging oxygen and an in plane oxygen (251) or the pseudo hollow site generated by two in plane O-atoms and one in the bridging rows (252) have the highest adsorption energy. Lopez and Nørskov have recently found in their calculations that the preferred adsorption site may depend on the coverage of gold which may help to explain differences between various theoretical predictions (253). Overall, gold was found to show much weaker bonding to titania when compared to copper or silver regardless of the adsorption site. Furthermore, as a consequence of the weak interaction, the energy differences between various sites are often very small (250). This can be explained by gold's relatively high electronegativity and high ionization potential which make gold a poor electron donor. Wang and Hwang found that one must apply caution in prediction of nucleation sites, particularly with regard to the size of the unit cell chosen, so as to capture the interaction between $\mathrm{Au}$ and the substrate instead of $\mathrm{Au}-\mathrm{Au}$ interactions (251).

Not surprisingly, defects were found to play an important role in the adsorption of gold on titania and magnesia. Lopez and Nørskov calculated that oxygen vacancies were about $0.5 \mathrm{eV}$ more stable than any possible adsorption site on the defect-free $\mathrm{TiO}_{2}$ (110) surface (253). Lopez et al and Giordano et al (249) also found that while sites along the (001) direction of rows containing $\mathrm{Ti}$ atoms are essentially energetically equal, diffusion along the protruding rows must overcome a large energy barrier. This result may help to explain why gold particles on $\mathrm{TiO}_{2}(110)$ are frequently elongated in a particular direction. Rodriquez et al (252) calculated that $\mathrm{Au}$ atoms adsorbed on oxygen vacancies were $48 \mathrm{kcal} / \mathrm{mol}(2.0 \mathrm{eV})$ more stable than the best possible adsorption site for the defect free surface. Wang and Hwang demonstrated with their DFT calculations that neighboring oxygen vacancies further increased the interaction between gold and the defect site (251). Yang et al. (254), and Vittadini and Selloni (255) calculated similar stability advantages for the adsorption of gold on oxygen vacancies on $\mathrm{MgO}(001)$ and $\mathrm{TiO}_{2}$ anatase (101) respectively. 
In addition to their work at low gold coverages, Madey's group has also examined the growth and nucleation behavior of large gold coverages on the $\mathrm{TiO}_{2}(110)$ surface (256). High resolution SEM images revealed that above a deposition of $1.5 \mathrm{~nm}$ (more than $5 \mathrm{ML}$ ), a new stage of growth began, marked by the elongation of particles and partial coalescence resulting in worm like islands. Continued deposition of gold resulted in decreasing island density due to coalescence, until the entire network of islands was connected at about an $8 \mathrm{~nm}$ coverage. Continued deposition eventually results in a granular gold film at an average thickness of $12 \mathrm{~nm}$. Madey and coworkers observed that upon annealing of the large worm like islands, the particle density was unchanged but the islands thickened and formed faceted structures, revealing more of the $\mathrm{TiO}_{2}$ surface underneath. This behavior is consistent with the island thickening model supported by Campbell (228) for metals grown on metal oxides. This is in contrast to the effect of annealing at lower coverages where both coalescence and ripening processes led to a decline in particle density simultaneous to the thickening process.

Although highly faceted particles have not been seen by STM for small gold particles on $\mathrm{TiO}_{2}$ in the same way as for $\mathrm{Pd} / \mathrm{Al}_{2} \mathrm{O}_{3}$ for example (257), HREM images of larger particles have shown that gold particles will grow in a semi-epitaxial manner $(236,258,259)$. Particles with a $\mathrm{Au}(111)$ orientation have been observed to grow such that gold lies on top of bridging row oxygen atoms. Along the (001) direction of $\mathrm{TiO}_{2}$, the lattice mismatch is only $2.6 \%$, but in the perpendicular (110) direction the misfit between two bridging oxygen rows and four Au atoms in (110) closed packed rows is $14.3 \%$. A more favorable arrangement with $\mathrm{Au}(112)$ particles growing atop $\mathrm{TiO}_{2}(110)$ has also been observed with Au atoms sitting atop 5-fold coordinated Ti atoms. The mismatch in the (001) direction is the same as before, but the mismatch in the (110) direction is now reduced to $8.4 \%$.

In Figure 13 the epitaxial relationships are displayed. Cosandey and co-workers found that when gold was deposited at $300 \mathrm{~K}$ and then annealed to $770 \mathrm{~K}$ the $(111)_{\text {Au }} / /(110)_{\text {Tioz }}$ orientation was preferred while direct deposition at high temperature preferentially gave rise to the (112)Au//(110) Tioz orientation (259). Interestingly, the Au lattice does not appear to undergo any deformation in spite of the minimal strain that must be overcome to match the $\mathrm{TiO}_{2}$ epitaxy, indicating that the interaction between gold and titania is rather minimal. XPS measurements confirmed this lack of chemical interaction as Ti $2 \mathrm{p}$ and $01 \mathrm{~s}$ did not show any core level shifts after deposition of gold for coverages above $3 \mathrm{ML}$ (227).

On the anatase phase of titania the situation is somewhat different. HREM have revealed a strong epitaxial relationship between gold and the support due to the small misfit (0.9\%)

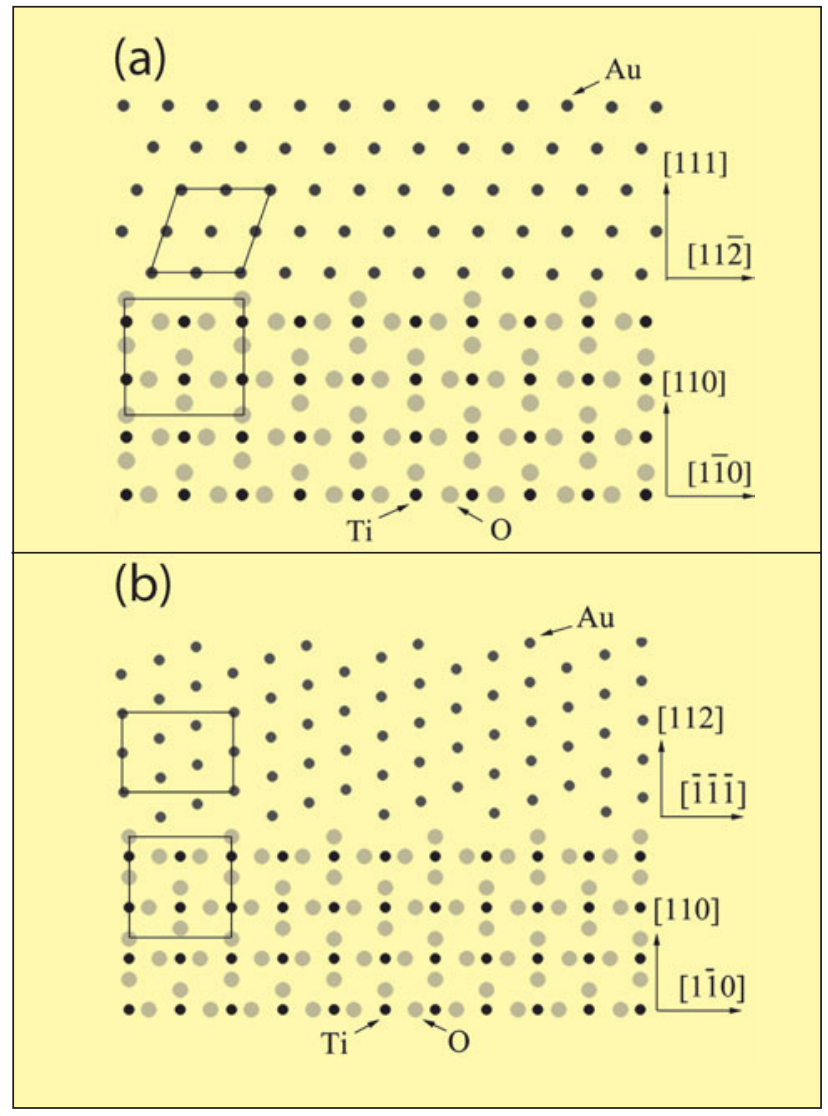

Figure 13

Cross-sectional representation of $\mathrm{Au} / \mathrm{TiO}_{2}(110)$ interface viewed along (001) TiO 2 direction with (a) (111) $\mathrm{Au}_{\mathrm{Au}} /(1110) \mathrm{TiO}_{2}$ and (b)

(112) ${ }_{\mathrm{Au}} / /(110) \mathrm{TiO}_{2}$ orientations. (258)

between the $\mathrm{Au}(111)$ face and the $\mathrm{TiO}_{2}(112)$ plane (260). Although small gold particles have been theoretically predicted to favor icosahedral and decahedral shapes (206$210,261)$, the relationship between titania and gold is strong enough such that particles can grow in a Au(111) epitaxy up to rather large sizes (258). Giorgio and co-workers have shown with HREM that such a relationship also exists for small Au particles on anatase (110) (262). Comparing experimental data with simulated images, the best match was found when the particles were assumed to be strained such that their lattice constant was deformed to coincide with the support. The $\mathrm{Au}(110)$ was therefore assigned to grow along the $\mathrm{TiO}_{2}(110)$ face such that the Au-Au distances are contracted by $5.3 \%$ along the Ti rows and expanded by $12.5 \%$ between the Ti rows. Such a strong relationship was found to continue up to the fourth layer of gold.

The thermal stability of Au particles on $\mathrm{TiO}_{2}$ has also been examined. Using HRSEM, Zhang et al observed substantial agglomeration of particles formed from a $3 \mathrm{ML}$ dose of gold (5 nm average diameter) when annealing at $775 \mathrm{~K}$ (240). However, no encapsulation of gold by $\mathrm{TiO}_{\mathrm{x}}$ under reducing or annealing conditions occurred, as has been observed for 


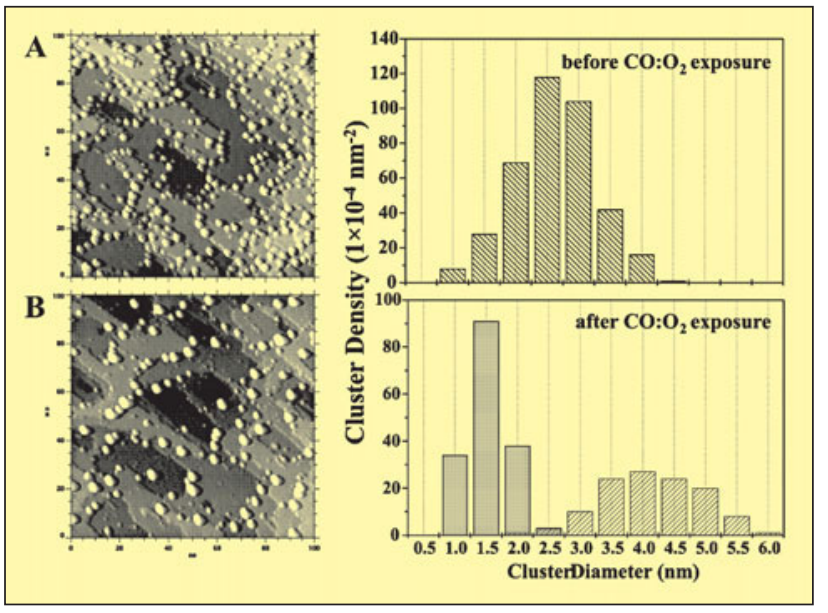

\section{Figure 14}

STM images and particle size distribution of the $\mathrm{Au} / \mathrm{TiO}_{2}(110)$ surface (a) before and (b) after $\mathrm{CO}: \mathrm{O}_{2}$ exposure (10 Torr, 2:1) showing Ostwald ripening. Images are $100 \times 100 \mathrm{~nm}^{2}$. (271)

platinum (263) and palladium (264) particles as a consequence of strong metal support interaction effects, so migration of gold into the oxide is not believed to occur. Previously Goodman's group had observed that gold particles deposited on $\mathrm{a} \mathrm{TiO}_{2}$ thin film at $120 \mathrm{~K}$ experienced substantial sintering between $120-400 \mathrm{~K}$, but then reached a stable plateau that continued up to $800 \mathrm{~K}$ (265). Kitchin et al recently showed STM images that revealed significant sintering upon annealing of gold particles on $\mathrm{TiO}_{2}(110)$ to $773 \mathrm{~K}$ (266). The particle density was seen to drop dramatically and the average particle diameter increased from $2.7 \mathrm{~nm}$ to $5.5 \mathrm{~nm}$ while the particle height increased from $0.45 \mathrm{~nm}$ to $0.75 \mathrm{~nm}$. Kitchin et al also reported that the particles (after annealing) appeared to be strongly associated with defects on the $\mathrm{TiO}_{2}$ surface.

Egdell and co-workers observed dramatic Ostwald ripening in their examination of the high temperature stability of small gold particles on $\mathrm{TiO}_{2}(110)$ with STM (267). Simply said, Ostwald ripening is a growth/loss process by which large particles grow at the expense of small particles $(268,269)$ usually resulting in a bimodal distribution. After deposition of $0.15 \mathrm{ML}$ of gold at room temperature a fairly narrow particle size distribution centered around $27 \AA$ was seen. After annealing the surface for seven hours at $750 \mathrm{~K}$, the particle size distribution had a bimodal form with a center at $2 \mathrm{~nm}$ and a second center at $4 \mathrm{~nm}$. The authors concluded that the Ostwald ripening must result from transport or migration of gold atoms from the edges of existing particles or which had not previously been incorporated into other clusters. This may be related to the low melting temperature of gold when in the form of small particles (270). Coalescence between particles at $750 \mathrm{~K}$ was also clearly observed in STM "movies". Although both of these growth processes may lead to catalyst deactivation it is difficult to assess which of these processes is dominant at the conditions of reaction.

Perhaps even more important than the initial nucleation and growth behavior of gold or its behavior under annealing conditions, is the behavior of supported gold under reaction conditions. Gold particles on titania were found to experience a form of Ostwald ripening when exposed to 10 torr of oxygen at room temperature (271).

Ripening in a reactive environment of $\mathrm{O}_{2}$ and $\mathrm{CO}$ (10 torr, 2:1 $\left.\mathrm{CO} / \mathrm{O}_{2}\right)$ has proved to be even stronger. Clusters with rather narrow size distribution centered around a diameter of $2.6 \mathrm{~nm}$ and a height of $0.7 \mathrm{~nm}$ were observed to grow to 3.6 $\mathrm{nm}$ in diameter and $1.4 \mathrm{~nm}$ in height as shown in Figure 14. Furthermore, the presence of several smaller clusters of less than $1.5 \mathrm{~nm}$ in diameter was observed. Interestingly, when larger particles with an average diameter of $4.2 \mathrm{~nm}$ were exposed to 10 torr of oxygen at room temperature, essentially no ripening was observed. This indicates the ripening processes are connected intimately with the reactivity of the particles. The mechanism of the ripening process is currently unknown, although speculations surrounding the formation of a volatile oxide, as in the case of silver (272), seem unlikely. Willneff et al have recently presented evidence that when $\mathrm{Au}_{2} \mathrm{O}_{3}$ was exposed to $\mathrm{CO}$ at $400^{\circ} \mathrm{C}$, an unidentified volatile species formed that is capable of transporting $\mathrm{Au}$ (273) although the oxide alone did not volatilize. When the surface of $\mathrm{TiO}_{2}(110)$ was pre-roughened with oxygen before gold deposition, the particles exhibited an enhanced stability in an oxygen atmosphere, possibly due to stronger binding of gold to surface irregularities. Gold does not seem to support the same type of titania regrowth that has been observed for palladium particles on $\mathrm{TiO}_{2}(110)$ (274).

Of course the next step in observation of the sintering behavior of gold under reaction conditions is an in-situ measurement. Kolmakov and Goodman have examined gold on $\mathrm{TiO}_{2}(110)$ in a reactive environment of $\mathrm{CO}$ and $\mathrm{O}_{2}$ with STM $(275,276)$. An initially fairly uniformly sized group of particles was observed to undergo severe Ostwald ripening at $720 \mathrm{~Pa}$ and $450 \mathrm{~K}$. As expected, increased surface temperature accelerated the ripening process. The authors suggested that the presence of oxygen served to weaken Au-Au bonds and thereby promoted sintering. Images taken under reaction conditions showed that the response of particles that were initially the same size could be quite different, as some particles decreased in size or even disappeared, while others seemed to remain stable. This result emphasizes the importance of the local environment in the structure and ultimately the reactivity of the particles.

AFM measurements by Iwasawa's group revealed significant sintering of $\mathrm{Au}$ particles on $\mathrm{TiO}_{2}(110)$ under an 
ambient atmosphere at temperatures as low as $363 \mathrm{~K}$ (277). However, pretreatment of the surface with ultraviolet radiation before deposition of gold resulted in a heightened resistance to agglomeration. The authors speculated that this behavior resulted not from surface defects but rather from the presence of hydroxyl groups which formed as a result of photo induced dissociation of water on the titania surface. The hydroxyl groups were suggested to react strongly with the goldphosphine precursor used by Iwasawa and thereby brought about the formation of highly stable small gold particles which showed limited agglomeration even at $493 \mathrm{~K}$ in air.

In an ex-situ experiment of gold on polycrystalline titania, Sykes et al observed extreme sintering of gold when exposing their sample to air or oxygen at room temperature for long periods (20 hours) (278). They concluded that the role of oxygen was to remove vacancies, which would otherwise bind the gold particles to the surface more strongly. Both Ostwald ripening and coalescence are thought to contribute to particle sintering.

Although most of the work on the growth, nucleation, and thermal stability of gold has been performed on the $\mathrm{TiO}_{2}(110)$ surface, gold has been studied on a variety of other oxides. Early TEM work by Poppa and others demonstrated that gold particles on mica (279-281), MgO (282-287) and sapphire (288) were quite mobile and that vapor deposited particles would move rapidly on the surface in an attempt to find a favorable epitaxial orientation such as $(111)_{\mathrm{Au}} / /(001)_{\mathrm{MgO}}$ or $(110)_{\mathrm{Au}} / /(110)_{\mathrm{MgO}}$. Green et al found that particles grew epitaxially on vacuum cleaved MgO crystals, but that on air cleaved crystals the orientations were random until thick (100 A) films were grown (283). Increases in the particle density were seen when the $\mathrm{MgO}$ substrates were sputtered with $\mathrm{Ar}$ ions prior to gold deposition as expected (286). However, attempts to induce similar nucleation sites with an electron beam only resulted in an increased nucleation density on $\mathrm{MgO}(111)$ and not on $\mathrm{MgO}(100)$, where an unexplained reduction in the nucleation density was observed.

More recently Henry's group has re-examined growth of gold particles on mica $(289)$ and MgO $(290,291)$ with AFM. Large particles grown on mica exhibit the $\mathrm{Au}(111)$ face as shown in Figure 15 with a very flat topography (289). The 23 $x \sqrt{3}$ reconstruction has not been observed on the top facets, although this is probably because the long-range periodicity of the superstructure is large compared $(6.5 \mathrm{~nm})$ to the size of the particle (22 nm average). The sides of such flat hexagonal or triangular particles displayed a lattice constant characteristic of the $\mathrm{Au}(100)$ surface. Gold particles deposited on $\mathrm{MgO}$ microcubes were found to grow in a (001) Aul/(001) $_{\text {MgO }}$ epitaxial relationship and exhibited fcC structures up to $4 \mathrm{~nm}$ with a expanded lattice parameter of $2.9 \%$ (290). Particles of greater than $4 \mathrm{~nm}$ diameter were seen to adopt a (111) Aul $/(100)_{\text {Mgo }}$ epitaxy as well, but the lattice constant observed in the larger particles was expanded to that of bulk gold regardless of orientation. Ajayan and Marks had previously observed that the structure and orientation of very small gold particles ( $\mathrm{d} \sim 1 \mathrm{~nm}$ ) can fluctuate significantly even at room temperature as the energy differences between conformations is on the order of $\mathrm{kT}$ as has been seen in calculations for gas phase clusters (292). However, the authors explain that the substrate can play a critical role in the stabilization of the particle in particular states. Giorgio et al ascribe their lack of observation of such transitions between structure, known as "quasimelting", to the high interfacial energy which must be overcome when the particles are epitaxially oriented. On $\mathrm{MgO}(100)$, Højrup-Hansen et al examined the nucleation behavior of gold and found that as the deposition time increased, the cluster density rapidly reached a saturation plateau (291). For long deposition times, the cluster density began to decrease once again due to coalescence. The saturation density was found to decrease with increasing temperature and by means of an Arrhenius relationship determine an activation energy for nucleation to be $0.12 \mathrm{eV}$. The authors concluded that since their results could not be described by a homogeneous nucleation mechanism, nucleation on point defects must be the prevailing process.

Robins and Rhodin found that the nucleation rate was directly proportional to the deposition rate leading them to conclude that nucleation was dominated by point defects (293). Furthermore, their observation that the nucleation density reached a constant maximum regardless of deposition rate or substrate temperature was presented as additional proof that the nucleation process was defect mediated. However, this result is in contrast with Henry's finding of an Arrhenius type relationship between nucleation density and substrate temperature. In Robins and Rhodin's model, point defects acted as perfect sinks for nucleation but recent theoretical (294) and experimental (295) work has shown that this does not apply for high substrate temperatures. The authors were able to observe a number of different types of defects on the MgO surface, all of which acted as nucleation centers for gold (296).

In their study of a gold particle grown on a MgO(100) step, Kizuka and Tanaka saw that the particle could rearrange continuously to maintain the lowest energy structure when holding the sample substrate at room temperature (297). Examining the ratio of $\mathrm{Au}(100)$ facets to $\mathrm{Au}(111)$ facets, repetitions in the truncation pattern were seen to emerge as the cluster grew until reaching a size of over 1500 atoms at which point rigid epitaxy with the support is lost. Pauwels and co-workers found in their examination of HREM images that both the MgO surface layers and the $\mathrm{Au}(100)$ layers were strained around the interface in an effort to match lattice constants (298). Interestingly, the lattice spacing at the 
edges of the particle were expanded to compensate for the compression of the lattice constant at the particle interior. In their examination of Au on MgO using TEM, Blick and coworkers found that gold grew two-dimensionally at low coverages forming thin rafts before exhibiting more three dimensional growth at higher coverages of gold (299).

Growth of gold on silica thin films has been studied by Luo et al (300). Similar growth behavior to $\mathrm{Au} / \mathrm{TiO}_{2}(110)$ was observed as the growth mode switched from 2-D to 3-D at approximately $0.1 \mathrm{ML}$. For gold deposited at $100 \mathrm{~K}$, sintering was observed to be strong when annealing the sample to $600 \mathrm{~K}$. However, above $600 \mathrm{~K}$ sintering slowed until gold was finally desorbed from the surface at above $1000 \mathrm{~K}$. When comparing LEIS data for the $\mathrm{Au} / \mathrm{SiO}_{2} / \mathrm{Mo}(110)$ model catalyst with the $\mathrm{Au} / \mathrm{TiO}_{2} / \mathrm{Mo}(100)$ model catalyst, in both cases the Au signal lost about half its intensity between $100 \mathrm{~K}$ and 800 $K$, indicating diffusion behavior of gold is essentially the same for both surfaces $(265,300)$.

However, unlike on $\mathrm{TiO}_{2}(110)$, growth of gold on thin alumina films has been seen by ourselves and others to be 3D even at very low coverages (301,302). Well ordered alumina films can be grown through the oxidation of the surface of a $\mathrm{NiAl}(110)$ single crystal. The thin alumina film, which we have studied extensively, exhibits large terraces, on which line and point defects exist, which may act as nucleation centres during metal deposition. STM images of gold deposited at $300 \mathrm{~K}$ showed that gold particles are randomly distributed on the surface. Based on previous extensive studies on metal nucleation and growth on alumina (233-235), the absence of preferential nucleation for deposition at $300 \mathrm{~K}$ indicates that the gold atoms have a fairly low mobility on the alumina surface. Even at a coverage of $0.05 \mathrm{ML}$ of gold, particles were seen to exhibit a hemispherical particle shape, indicating 3-D growth from the onset. The Au particles formed on alumina showed a narrow size distribution. In addition, the system morphology was quite stable towards heating up to $600 \mathrm{~K}$ : the particles only slightly sintered as evidenced by STM and LEED measurements.

Carrey et al have proposed that large gold particles on an amorphous alumina film can be more mobile than single atoms or small clusters $(303,304)$. The authors observed that the nucleation density of gold was not affected strongly by deposition temperature leading them to conclude that the nucleation must be dominated by defects. Furthermore, the deposition resulted in a bimodal size distribution, indicating that particles above a certain size could more easily diffuse and coalesce during deposition while smaller particles were trapped on defects.

The thin FeO(111) $(305,306)$ film grown on a Pt(111) single crystal has only recently begun to attract attention as a suitable oxide substrate for model metal/oxide systems

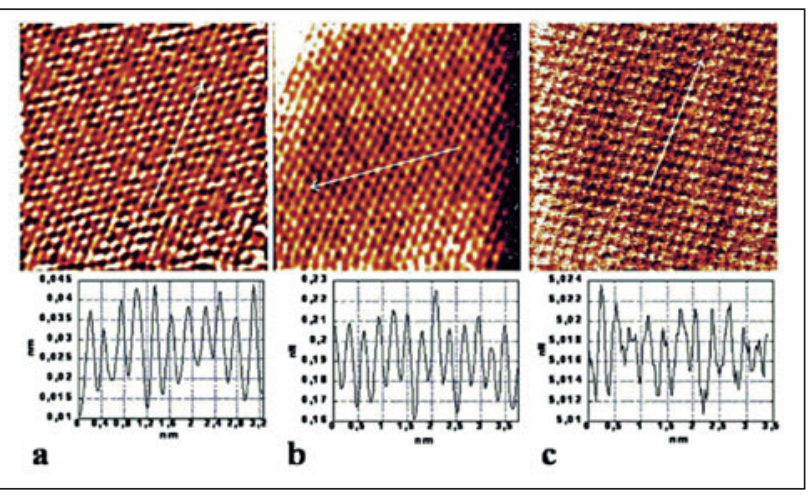

\section{Figure 15}

(a) AFM topographs (b) lateral force and (c) normal force images of the top face of a flat gold particle formed on mica. (Image size $6 \times 6$ $\left.n m^{2}\right) \cdot(289)$

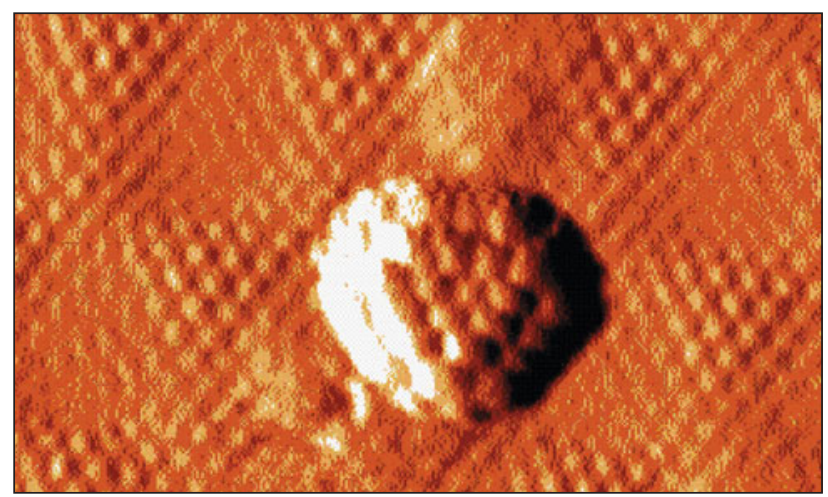

\section{Figure 16}

High resolution STM image of a monolayer gold particle formed on a thin $\mathrm{FeO}(111)$. (Image size $10 \times 6 \mathrm{~nm}^{2}$ ).

(302,307). The oxide layer exhibits an O-Fe-Pt-Pt- stacking and strongly relaxed surface, with $\mathrm{O}$-Fe and Fe-Pt distances being $\sim 0.6$ and $\sim 1.8 \AA$, respectively. The surface shows a characteristic superstructure, clearly visible in STM and LEED, formed by a 10\% mismatch between FeO(111) and Pt(111) lattices (305).

Gold deposition first resulted in decoration of steps and "holes" present in oxide film, which typically cover less than $3 \%$ of a monolayer. Subsequently, small Au clusters started to nucleate and grow at the terraces as well. At high coverages, Au aggregates were essentially homogeneously distributed on the FeO(111) surface. The nucleation density of gold on the FeO film was approximately half that of gold on $\mathrm{Al}_{2} \mathrm{O}_{3} / \mathrm{NiAl}(110)$. This implies that, albeit both oxides are very thin $(<5 \AA)$ and terminated by oxygen, gold diffusivity on these supports is quite different. The higher diffusivity observed for gold on FeO probably results from a lower defect density on the FeO film relative to alumina.

The high resolution STM image presented in Fig. 16 shows a $1.5 \mathrm{~nm}$ diameter island of just a single (111) layer which is preferentially formed for $\sim 0.1 \AA$ A-thick Au overlayer. As both 
the support and Au island are atomically resolved, it is clear that the gold layer is in good epitaxial relationship with the oxide support. With increasing amounts of gold, threedimensional particles up to $7 \mathrm{~nm}$ in diameter and $1.5 \mathrm{~nm}$ in height could be grown (for 2 A-thick overlayer). Since good epitaxy with support was observed for Au monolayer islands, it seems likely that large Au particles grow such that they expose (111) top facets, as has been observed for gold particles on mica (289).

We have extended the studies to other iron oxide films. Previous studies of the $\mathrm{Fe}_{3} \mathrm{O}_{4}(111)$ films formed on $\mathrm{Pt}(111)$ have shown that the regular surface is terminated by $1 / 4$ of a monolayer (with respect to oxygen sub-layer) of $\mathrm{Fe}^{3+}$ cations (306). Besides terrace steps, the defect structure, which may influence the nucleation and growth of Au particles, includes iron vacancies and, in some cases, small domains exhibiting "biphase ordering", whose presence at low concentration can be only observed with STM $(308,309)$.

Accordingly, the gold diffusion on $\mathrm{Fe}_{3} \mathrm{O}_{4}(111)$ was found to be limited relative to $\mathrm{FeO}$, thus preventing sintering at $300 \mathrm{~K}$. However, heating to higher temperatures promoted sintering such that particles annealed to $500 \mathrm{~K}$ grew and displayed better faceting (302). The histogram analysis of STM images for annealed samples revealed that height of the particles was found to be by multiples of $2.5 \AA$ (equal to the height of a monolayer of gold in the $\mathrm{Au}(111)$ orientation) which implies that particles grow by increasing the number of the layers in the cluster. In addition, the annealed particles are well ordered exhibiting mostly hexagonal (and trigonal) shape. It seems plausible that the top facets of the particles show the (111) surface owing to small ( 3\%) lattice mismatch between $\mathrm{Au}(111)$ and $\mathrm{Fe}_{3} \mathrm{O}_{4}(111)$ surfaces.

The STM results for the $\alpha-\mathrm{Fe}_{2} \mathrm{O}_{3}(0001)$ film are very similar to those found on $\mathrm{Fe}_{3} \mathrm{O}_{4}$ films. Once again, 3-D Au particles are found homogenously distributed on the oxide surface, and they exhibit a hemi-spherical shape. Previously, Haruta found by TEM that gold forms hemispherical particles on $\mathrm{Fe}_{2} \mathrm{O}_{3}$ with an epitaxial orientation of (111) Au//(110) $\mathrm{Fe}_{2} \mathrm{O}_{3}$ (310). However, the $\mathrm{Fe}_{2} \mathrm{O}_{3}$ surface termination is critical to the preparation conditions (306), and therefore the nucleation behavior is currently a subject of investigation.

\subsection{Electronic properties of supported $\mathrm{Au}$ particles}

It is well known that small particles supported on oxides can exhibit electronic, optical, and magnetic characteristics different from bulk materials (311-316). In the case of gold, this is especially important as several researchers believe it is precisely these changes in the electronic character that give rise to its unique catalytic properties. Although gold catalysts are not thought to exhibit Strong Metal Support Interactions (317), at least in the classical sense (318), perhaps the role of the

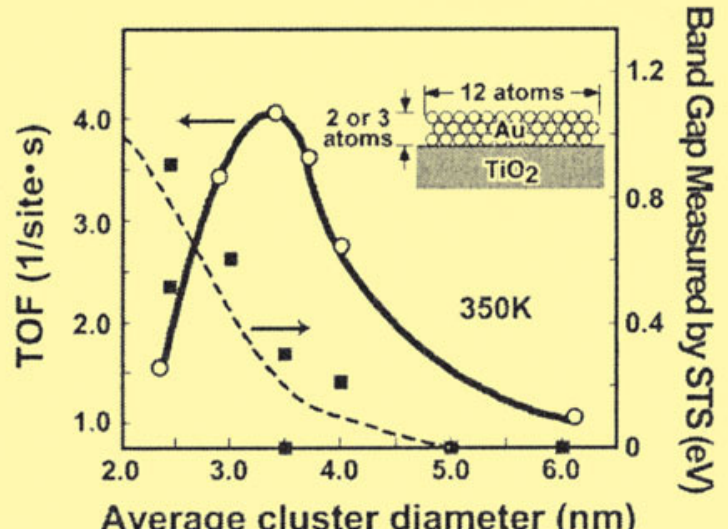

Average cluster diameter $(\mathrm{nm})$

\section{Figure 17}

Turnover frequency and band-gap measured by STM/STS as a function of the diameter of $\mathrm{Au}$ islands formed on $\mathrm{TiO}_{2}$. (2 (data from 192))

support is to influence the electronic nature of the gold through charge transfer. Aside from specific support effects, quantum size effects $(319,320)$ are also frequently invoked to explain the exceptional activity of gold nanoparticles. Valden et al demonstrated with Scanning Tunneling Spectroscopy that the maximum in activity for Au particles on $\mathrm{TiO}_{2}(110)$ coincided with the onset of an appearance of a band gap, marking a transition point from bulk metal to a semi-conductor (192) as shown in Figure 17. At a diameter of $3.2 \mathrm{~nm}$ and height of 1.0 $\mathrm{nm}$ ( 300 atoms) a band gap of 0.2-0.6 eV can be measured which increases up to $1.4 \mathrm{eV}$ were measured for the smallest particles $(\mathrm{d}<2.0 \mathrm{~nm})$. Valden et al asserted that the gold particles experience this unusual activity as a consequence of their reduced dimensionality and suggested that particle thickness may be the key parameter. Similar measurements of band gaps for Au particles on HOPG indicated that the metalnon-metal transition took place at even smaller particle sizes ( $1 \mathrm{~nm})(321)$. However, this could be explained by differences in geometry between particles on various supports as small gold clusters on HOPG were found to be spherical (322).

In their study of acrolein hydrogenation over $\mathrm{Au} / \mathrm{TiO}_{2}$ and $\mathrm{Au} / \mathrm{ZrO}_{2}$ catalysts, Claus and co-workers also invoked quantum size effects to explain a change from the preferential hydrogenation of $\mathrm{C}=\mathrm{O}$ to $\mathrm{C}=\mathrm{C}$ as the particle size decreased below 2 nm (323). Small gold particles sitting above oxygen vacancies of the oxide may be electron rich due to charge transfer from the support and would therefore be predicted to experience a repulsive interaction with the $\mathrm{C}=\mathrm{C}$ bond. However, due to the quantum size effects, the $d$ band of a small gold particle will possess a decreased density of states which lowers the repulsive interaction. At the same time, the depletion of the $d$-band also results in a loss of back donation into the $\mathrm{C}=\mathrm{O}$ and thus favors the hydrogenation of a $\mathrm{C}=\mathrm{C}$ bond over a $\mathrm{C}=\mathrm{O}$ bond. 
Therefore since the activity of gold has been linked to the transition from metallic to non-metallic particles, the exact point at which this transition occurs is the subject of much interest. Using size selected clusters supported on amorphous carbon, Berry and co-workers found that $\mathrm{Au}_{33}$ (and smaller clusters $\mathrm{Au}_{27}, \mathrm{Au}_{7}$, and $\mathrm{Au}_{5}$ ) showed significant shifts in both the valence and core regions by XPS (324). The authors correlated these shifts with the average coordination number of the atoms in the cluster. From an extrapolation of their data, the transition from metallic to non-metallic behavior was predicted to occur at around 150 atoms. In a similar examination based upon the $5 d$ band splitting, Lee et al had estimated that this transition should take place at a particle diameter of more than $1.9 \mathrm{~nm}$, which corresponds to a hemispherical particle with over 100 atoms (325). Using HREELS, Guo et al. estimated that this transition occurred for gold on $\mathrm{TiO}_{2}$ (110) at a coverage of $0.2 \mathrm{ML} \mathrm{Au}$, which corresponds to particles of about $2 \mathrm{~nm}$ in diameter and 2 atomic layers high (242). For gold on a thin alumina film, the transition was not seen until a coverage of $0.3 \mathrm{ML}$ of $\mathrm{Au}$ (242). Whetten and co-workers found that discrete states in the optical absorption spectra began to emerge at diameters below 2 nm ( 200 atoms) (326). The authors noted that this transition took place at the same point where a structural transition occurs, suggesting the two phenomena may be related (207). Stievano et al employed Mössbauer spectroscopy to observe changes in the electronic character of small gold particles supported on Mylar as the dimension was shrunk (327). Measurements revealed an asymmetry as the particle size decreased below a $1.5 \mathrm{~nm}$ diameter, due to the increased fraction of surface atoms. A decrease in the ion splitting was explained by an electron transfer from the core to the surface atoms. Recently, Boyen et al showed by XPS that Au particles on diamond displayed the valence band spectra as bulk gold down to a diameter of $1.6 \mathrm{~nm}(\sim 100$ atoms) (328). Lin et al used the field emission energy distribution to observe the metal-non-metal transition for gold (329). Particles below $3.0 \mathrm{~nm}$ (approximately 800 atoms) were observed to display discrete electron energy states as opposed to the continuum of states in bulk gold. Calculations based on an electronic shell model were in good agreement with the experimental observations.

Such examinations underline the importance of photoelectron spectroscopy in studying the electronic character of small gold particles. Core level binding energy shifts can be interpreted as initial state effects which result from quantum size effects inherent to the particles themselves and final state effects which result from relaxation processes and are strongly connected to the support $(234,330)$. Of course, smaller particles also have more surface atoms, which due to their decreased coordination results in a shift of the $d$-band toward the Fermi level and a corresponding decrease in the binding energy of the core electrons. In the case of gold, Wertheim and coworkers have measured this surface state shift to be $\sim 0.4 \mathrm{eV}$ (331). For small unsupported gold clusters Häberlen et al. calculated that shifts to lower binding energies can range up to $1.0 \mathrm{eV}$ for $\mathrm{Au}_{13}$ clusters and that even $\mathrm{Au}_{147}$ clusters show an overall shift of $0.4 \mathrm{eV}$ (206). Furthermore it is possible to distinguish between initial and final state effects through analysis of the so-called Auger parameter (332) by which one can determine to what extent the relaxation effects play a role in the binding energy shift by the creation of a double hole. Photoemission experiments are also useful in observation of charge transfer from the support to the particle (or vice-versa).

Mason attributed positive binding energy shifts observed for $\mathrm{Au} / \mathrm{SiO}_{2}$ and $\mathrm{Au} / \mathrm{C}$ to an initial state effect arising from changes in the electronic configuration of small particles due to rehybridization of the valence band (333). Mason asserted the relative similarity in the binding energy shift for gold on two very different substrates was strong evidence for the importance of initial state effects. In a similar comparison, Liang et al examined the valence spectra of gold deposited on aluminum and aluminum oxide and saw similar behavior with respect to the position of the $d$-band and the degree of $d$ band splitting on both substrates (334). This led the authors to conclude that the deviations from bulk behavior must arise from intrinsic electronic properties of the small particles.

In contrast, Wertheim et al concluded that such positive binding energy shifts must arise from final state effects from their comparison of XPS spectra of Au particles on vitreous carbon (non-conducting) and Metglas (conducting) (335). A slight negative core level binding energy shift was observed in the latter case, even though the characteristic narrowing of the $d$-band was observed in the valence spectra indicating the formation of small particles. Kim and Winograd used implantation of $\mathrm{Ar}$ into gold and gold supported on $\mathrm{SiO}_{2}$, as a way to compare the chemical environment of gold in the bulk with gold on $\mathrm{SiO}_{2}$ (336). The positive binding energy shift of Au $4 f$ for small gold particles on silica was interpreted as a final state effect as Ar was found to undergo a shift of similar magnitude when compared to Ar implanted in a bulk gold sample.

Although Coulthard et al acknowledged that their observation of positive binding energy shifts was consistent with the idea of hybridization effects endorsed by Mason (333), XANES measurements indicated that small gold clusters possess an enrichment of $d$ charge (337). As an initial state view would predict a $d$ count in between 9 (single atom) and 10 (bulk metal), this appears inconsistent with the XANES data. Since XANES experiments do not suffer from charging effects, one can conclude that the shifts observed by XPS must arise from final state effects. 
Of course, both initial state effects and final state effects will be present and several researchers have found that one must consider both effects to obtain an accurate picture for the origin of binding energy shifts. When Cordes and Harsdorff examined the $4 f$ region of gold particles supported on amorphous carbon, they found that the positive core level shift had disappeared for particles with a diameter greater than $1.5 \mathrm{~nm}$ (338). However, the valence band region did not exhibit bulk behavior until the particles were at least $3.5 \mathrm{~nm}$ in diameter. Thereby, the authors concluded that the same mechanism cannot be controlling the deviation from bulk behavior in the two separate energy regions. Cordes and Harsdorff hypothesized that while the core region spectra are dominated by relaxation processes, the valence band depletion was a result of decreased spin-orbit coupling in smaller particles. Roulet et al examined the valence region of gold particles from 1.5-6.0 nm on $\mathrm{NaCl}$ and also observed depletion near the Fermi Level as the particle size was decreased (339). Particles of $4.0 \mathrm{~nm}$ or less exhibited significant band narrowing as well. The band narrowing was interpreted as a consequence of reduced coordination.

In a comparison of particles with different morphologies, but approximately the same total number of atoms, Costanzo et al observed that two-dimensional gold particles grown on HOPG experienced smaller binding energy shifts as compared to more spherical particles deposited on amorphous graphite (340). While the positive binding energy shift was attributed to final state effects, the differences between particles were interpreted as initial state effects arising from the higher percentage of surface atoms in the particles on HOPG.

In Dalacu et al's examination of gold particles of various sizes on $\mathrm{SiO}_{2}$ supports with XPS, positive binding energy shifts for the Au $4 f$ peak of up to $0.5 \mathrm{eV}$ were observed for the smallest particles (341). After annealing, the shift increased to even higher binding energies (up to $1.3 \mathrm{eV}$ shift), which was interpreted to be primarily a final state effect resulting from decreased contact with the support with only a minor contribution by initial state effects due to a decrease in the number of exposed surface atoms.

In an examination of gold on $\mathrm{ZrO}_{2}$, Zafeiratos and Kennou used the Auger parameter to separate initial and final state effects (342). While final state effects dominated the overall binding energy shifts observed, it was possible to observe initial state effects reflecting the average coordination number up to a gold coverage of $1.5 \mathrm{ML}$. It should be remembered, however, in analysis of the Auger parameter that the relaxation comparison of the double hole and single hole should be from the same shell. Recently, in our own analysis involving copper and cobalt particles (343), we have obtained evidence that lattice contraction in very small particles can induce positive binding shifts due to hybridization as predicted by Mason (333). This situation is of particular importance to gold which has a strong tendency to undergo contraction in the surface layer (206,344-348), hence the reconstructions of $\mathrm{Au}(111)$ and $\mathrm{Au}(100)$.

In an investigation of support effects, Chusuei et al measured binding energy shifts of gold on $\mathrm{SiO}_{2}$ and $\mathrm{TiO}_{2}$ and saw rather large differences between the two substrates (0.8 eV for $\mathrm{Au} / \mathrm{TiO}_{2}, 1.6 \mathrm{eV}$ for $\mathrm{Au} / \mathrm{SiO}_{2}$ ) (349) as shown in Fig 18. Due to a lack of structural data for $\mathrm{Au} / \mathrm{SiO}_{2}$, it is not possible to say precisely how these effects originate. However, this data seems to indicate that interactions of the support with gold particles are present that must affect the electronic character of the particles. Band bending observed by shifts in the XPS for Ti $2 p(0.15 \mathrm{eV})$ for submonolayer coverages of gold on titania, give support to the notion that the positive binding energy shifts observed in the Au $4 f$ result from the interaction between gold and the oxide support (227).

Yang et al have calculated that the $\mathrm{TiO}_{2}$ support should induce negative binding energy shifts of 1.1-1.5 eV for a gold monolayer lying above it due to transfer of charge from the support to the particle (248). At first this result appears inconsistent with Chusuei et al (349). However, as the authors point out, Au particles on titania do have a negative core level shift as compared to $\mathrm{Au} / \mathrm{SiO}_{2}$ (which is a non-interacting support). Some researchers have invoked final state effects as an explanation for the discrepancy between theory and

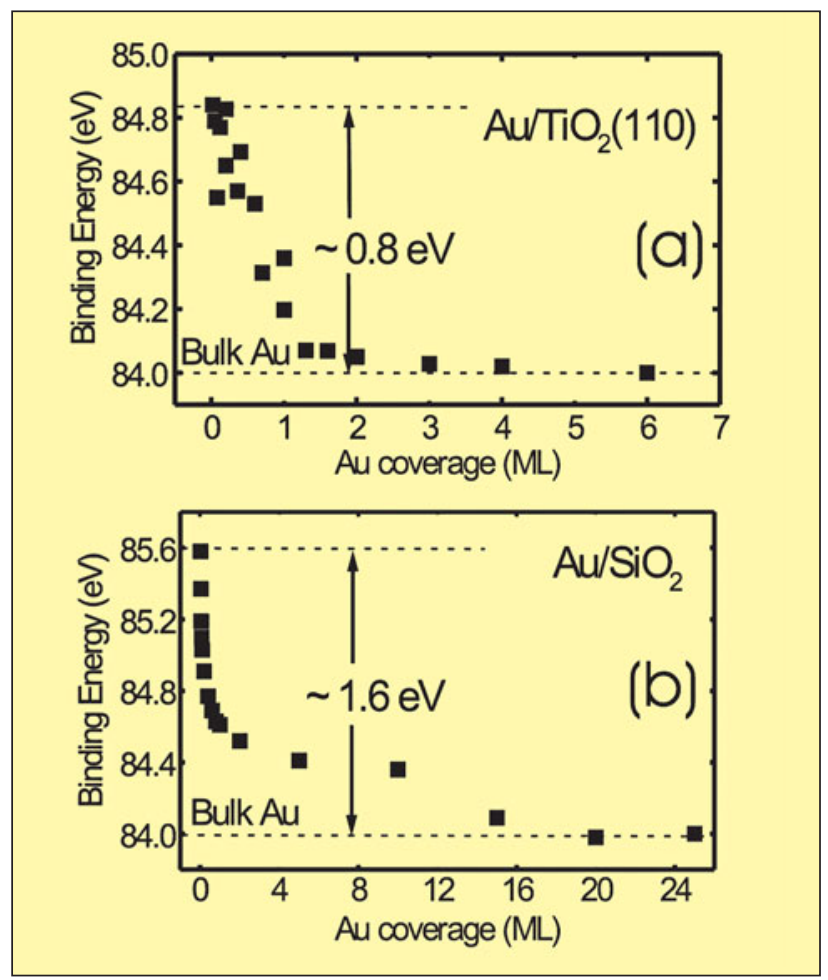

Figure 18

Plots of binding energy measured by XPS of the Au 4f7/2 core level as a function of Au cluster coverage on (a) $\mathrm{TiO}_{2}(110)$ and (b) $\mathrm{SiO}_{2}$ surfaces. (349) 
experiment. However, in recent DFT calculations it has been demonstrated that final state effects are insufficient to explain the positive binding energy shifts observed by Goodman's group and others. Yang and Wu explain the positive binding energy shifts observed through the interaction of gold with oxygen vacancies (350).

Wu and co-workers went on to perform calculations for adsorption of gold on $\mathrm{MgO}(001)$, taking a special interest in perturbation of the electronic structure by defects (254). Like on $\mathrm{TiO}_{2}(110)$, gold on $\mathrm{MgO}(001)$ was found to experience core level shifts to lower binding energies for adsorption on the regular surface. On defects (oxygen vacancies), the situation was reversed as gold was found to undergo a shift of $1.8 \mathrm{eV}$ to higher binding energies. These calculations seem at odds with XPS experiments for $\mathrm{Au} / \mathrm{SiO}_{2}$ and $\mathrm{Au} / \mathrm{TiO}_{2}$ showing that reduced surfaces give rise to negative shifts in the binding energy as one would expect for an anionic gold species $(351,352)$. Howard et al examined the influence of defects on the support upon the electronic character of gold particles (352). After bombardment of the $\mathrm{TiO}_{2}(110)$ surface with argon before gold deposition, only very small core level shifts were observed $(0.1 \mathrm{eV}$ at $0.5 \mathrm{ML} \mathrm{Au})$. Therefore the negative shift due to charge transfer from the $\mathrm{Ti}^{3+}$ states to gold balanced out the shift to higher binding energies due to final state effects that was observed for particles on the defect free surface. Even more dramatic, after deposition of gold on the reduced surface, the signal for $\mathrm{Ti}^{3+}$ was found to practically vanish. Examination of the valance band uncovered a strong depletion around the Fermi level for small particles due to either initial state effects or charge transfer to the substrate. On the defective surface an even greater shift in the onset of photoemission was observed, leading the authors to conclude that the depletion of intensity near the Fermi level resulted from an interaction between Ti $3 d$ defect states and gold. Vijay et al in their calculations of the growth of gold on titania found that the charge left behind by a vacancy was not isolated on the two exposed titanium atoms but rather was spread over a fairly large ensemble in the oxide (250). Therefore, one should consider that vacancies, which previously had been thought to be a highly localized phenomenon, may have long-range effects on the surface chemistry.

Goodman and co-workers found in their calculations a shift in the valence band for $\mathrm{Au} / \mathrm{TiO}_{2}$ towards the Fermi Level by $1.8 \mathrm{eV}$ for a single monolayer of gold (248). This shift in the $5 d$ band toward the Fermi level suggested that small supported gold clusters may not be so "noble" and should therefore have enhanced chemical adsorption properties as compared to bulk gold. Lopez and Nørskov found evidence in their calculations that this result was dependent upon the coverage of gold (253). For coverages of less than one monolayer, the $d$-band was shifted to the Fermi level by

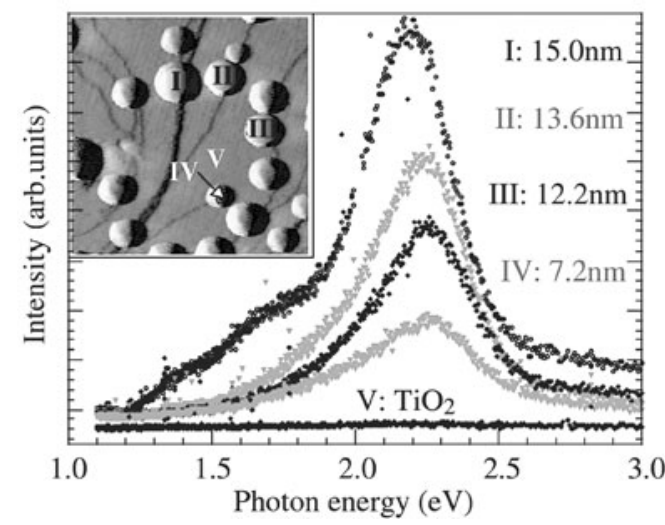

Figure 19

Photon-emission spectra of Au particles of various sizes on $\mathrm{TiO}_{2}(110)$ as marked in the STM image shown in the inset (size $130 \times 130 \mathrm{~nm}^{2}$ ). The emission is dominated by decays of Mie-like plasmons. (353)

upwards of $1.0 \mathrm{eV}$ when compared to bulk gold. However, such initial state effects were balanced by a strong interaction between gold and the underlying support, leading to a shift in the position of the $d$-band to higher binding energies with respect to a free gold cluster. As the coverage of gold was increased, the fraction of highly coordinated gold atoms in the particle increased, leading to more metal-metal bonding and thereby drawing electron density away from the particle-support interface. For coverages as low as two monolayers, the density of states was essentially identical to bulk gold.

Wang and Hwang also observed a shift upward in the DOS when Au was placed upon a titania surface, indicating there should be considerable changes in the chemistry of supported gold compared to bulk gold surfaces (251). Perhaps surprisingly, the strong interaction between gold and a defective $\mathrm{TiO}_{2}$ surface, actually serves to broaden the $5 d$ states of gold, possibly reducing the particle's reactivity. However, charge density plots also reveal some charge transfer from the defect site to Au.

In our own lab, we have examined the electronic properties of small gold particles using photoemission STM $(353,354)$. The Mie plasmon resonance energy of a cluster can be probed by injecting an electron into the cluster with an STM tip and analyzing the photons emitted. In a simple synopsis, the Mie plasmon is the collective oscillation of the conduction electrons in a particle relative to the core. The photoemission spectra of $7 \mathrm{~nm}$ diameter $\mathrm{Au}$ particles supported on a $\mathrm{TiO}_{2}(110)$ single crystal revealed that the electronic character of the particle was essentially unaffected by the support. The resonant photon energies were found to increase with decreasing particle size as the contribution of surface atoms with more localized $d$-electrons increases as 
shown in Figure 19, although particles in the non-metallic range could not be probed due to interference from tip induced plasmons. This is a consequence of the peculiar dielectric function of gold, which passes through a plateau region precisely in the range of the Mie plasmon resonance for gold and results in a heightened sensitivity of the emission to the local environment (i.e. the STM tip condition).

\subsection{Adsorption and reaction on supported Au nanoparticles}

In some of the earliest work, Haruta et al $(9,191,355)$ identified that the onset of activity of gold catalysts for $\mathrm{CO}$ oxidation could vary widely with the choice of support. Even in a comparison of different forms of titania (anatase, rutile, amorphous), large differences in reactivity can be seen (356). Some researchers ascribed this effect to differences in the particle size distribution on the various supports (although the gold loading was kept constant) (357,358). However, Grunwaldt et al showed a large difference in activity between zirconia supported gold catalysts and titania supported catalysts $\left(\mathrm{Au} / \mathrm{TiO}_{2}\right.$ >> $\left.\mathrm{Au} / \mathrm{ZrO}_{2}\right)$ even though the particle sizes were identical (359). The authors believe that the support affected the gold particles' ability to activate (dissociate) oxygen. The results were interpreted as differences between reducible and non-reducible supports. Behm and co-workers (226) found that the activity of gold catalysts supported on "non-reducible" metal oxide supports was considerably less than that of "reducible" metal oxide supports, as $\mathrm{Au} / \mathrm{Fe}_{2} \mathrm{O}_{3}$ and $\mathrm{Au} / \mathrm{TiO}_{2}$ were over five times more active than $\mathrm{Au} / \mathrm{Al}_{2} \mathrm{O}_{3}$. Furthermore, Behm's group reported that the activity of these inert supports was more sensitive to the size of the supported particles. The authors ascribed this difference in activity to a possible difference in the mechanism of oxygen adsorption. More recently, the oxidation of $\mathrm{CO}$ by gold supported on alumina and titania has been compared by Haruta and coworkers (360) and only slight differences in activity between gold catalysts were found when care was taken to ensure that the gold particles are well dispersed.

Supported gold particles have shown in numerous examples to exhibit heightened interaction with adsorbates as compared to single crystal surfaces. For example, using XPS, Rodriquez et al observed the dissociation of $\mathrm{SO}_{2}$ on 2.6 $\mathrm{nm}$ diameter gold particles on $\mathrm{TiO}_{2}$ (110), even though $\mathrm{SO}_{2}$ adsorbs only very weakly on the bulk surfaces of gold (252). While $\mathrm{SO}_{4}$ was found to be the only product on the clean $\mathrm{TiO}_{2}(110)$ surface, $\mathrm{SO}_{2}$ experienced total dissociation when in contact with gold on titania. XPS experiments also showed an increase in the relative fraction of $\mathrm{Ti}^{(4-x)+}$ after annealing to $750 \mathrm{~K}$ when gold was present as compared to its absence. The authors hypothesized that gold served to increase the rate of vacancy exchange between the surface and the bulk of $\mathrm{TiO}_{2}$, while at the same time gold is electronically modified by the presence of oxygen vacancies. Collaborative density functional theory calculations revealed that $\mathrm{SO}_{2}$ dissociation was greatly enhanced on gold when the particle is located above an oxygen vacancy.

Rodriguez and co-workers found further evidence of support effects in their comparison of $\mathrm{SO}_{2}$ adsorption on $\mathrm{Au} / \mathrm{TiO}_{2}$ as compared to $\mathrm{Au} / \mathrm{MgO}$ (361). In the case of $\mathrm{MgO}$ as a support, no dissociation of $\mathrm{SO}_{2}$ was observed, although the desorption temperature was much higher than that of $\mathrm{SO}_{2}$ from an Au single crystal. This would imply that small gold particles on $\mathrm{MgO}$ are a somewhat intermediate case: less active than $\mathrm{Au} / \mathrm{TiO}_{2}$ but more active than bulk gold surfaces. Since the Au particle size was expected to be about the same on both surfaces, Rodriguez et al attribute the differences observed to metal-support interaction effects.

As discussed in the previous section, the electronic character of gold may be perturbed by an interaction with the support. A consequence of the perturbation may be unique adsorption behavior that neither gold nor the support material exhibit alone. An example of this type of synergism is the dissociation of $\mathrm{H}_{2} \mathrm{~S}$ on $\mathrm{Au} / \mathrm{Cr}_{2} \mathrm{O}_{3}$ catalysts as studied by Rodriguez and co-workers with synchrotron radiation measurements (362). Ab initio calculations supported a mechanism whereby a charge transfer occurred from the gold particles to the oxide support and $\mathrm{H}_{2} \mathrm{~S}$ decomposed easily on the electron deficient gold.

Even when gold is deposited on a "non-interacting" support such as activated carbon, support effects can be seen. Bianchi et al observed differences in activity of various $\mathrm{Au} / \mathrm{C}$ catalysts for the oxidation of ethylene glycol depending upon the support although the particle sizes were essentially equal (363). The differences in activity were ascribed to presence of phenolic groups on the surface and their interaction with dispersed gold.

The synergism between gold and the support can also be observed by changes in the metal oxide. For example, it has been seen that certain metal oxides like $\mathrm{CeO}_{2}$ (364) and $\mathrm{Fe}_{2} \mathrm{O}_{3}(365)$ are more easily reducible in the presence of gold. Using ESR spectroscopy, Andreeva et al found that the $V=0$ bond length was lengthened in $\mathrm{Au} / \mathrm{V}_{2} \mathrm{O}_{5}$ catalysts after gold deposition leading to a higher delocalization of $3 d$ electrons and suggesting a strong interaction between $\mathrm{Au}$ and the support (366).

Given the complicated nature of the relationship between gold and metal oxide supports, it seems logical to begin our examination of size and support effects in gold catalysis with a discussion of the simple case of CO adsorption on gold model catalysts like those described in section 5.1. In Rainer et al's examination of the adsorption of $\mathrm{CO}$ on an $\mathrm{Au} / \mathrm{Al}_{2} \mathrm{O}_{3}$ thin film model catalyst with IRAS, CO was found to exhibit a single peak at $2100 \mathrm{~cm}^{-1}$ at $100 \mathrm{~K}$ for $0.5 \mathrm{ML}$ Au deposited at 300 K (367). In agreement with Ruggerio and Hollins work on 
$\mathrm{Au}(332)$ (83) this peak underwent a reduction in intensity and a blueshift of $11 \mathrm{~cm}^{-1}$ as the sample was heated to 200 $\mathrm{K}$. When higher gold coverages were used, a small blue shift to $2110 \mathrm{~cm}^{-1}$ was observed. The former effect is in contrast to the behavior of CO on other metal surfaces such as Pt (93) and $\mathrm{Pd}(94)$ and is ascribed to the unique electronic character of gold by which the back donation of electrons from gold into the $2 \pi^{*}$ orbital of CO is reduced (368). The latter effect is understood as a consequence of a decrease in the density of adsorbed CO as the particle size is increased. Winkler et al found similar results in their examination of $\mathrm{CO}$ on $\mathrm{Au} / \mathrm{Al}_{2} \mathrm{O}_{3} / \mathrm{NiAl}(110)$ using IRAS (369). In accompanying TPD experiments, CO was seen to desorb in two peaks at $105 \mathrm{~K}$ and at $145 \mathrm{~K}$ from gold particles of $\sim 10$ atoms. Attempts to observe oxygen desorption or $\mathrm{CO}_{2}$ production on these small particles failed.

In our work examining $\mathrm{CO}$ adsorbed on Au particles on an $\mathrm{Al}_{2} \mathrm{O}_{3}$ thin film, TPD experiments revealed two clearly resolved peaks, with the first peak at $120 \mathrm{~K}$ and another located at a higher temperature, depending on the nominal thickness of the Au overlayer (302). Bearing in mind that CO does not adsorb on the alumina film above $77 \mathrm{~K}$, observation of two desorption states may, in principle, indicate separate CO adsorptions on the metal and at the metal-oxide interface. However, based upon Ruggerio and Hollins observation of CO desorption from the stepped $\mathrm{Au}(332)$ single crystal surface (83) we conclude that both peaks could be attributed to desorption from gold particles. The position of the high temperature peak gradually shifts from $210 \mathrm{~K}$ to $160 \mathrm{~K}$ as the Au nominal thickness increases from 0.1 to $1.3 \AA$. This result clearly shows a particle size effect such that small $\mathrm{Au}$ particles adsorb CO more strongly.

The intensity of $\mathrm{CO}$ desorption signal reached saturation at a fairly low coverage of gold, as the spectra are nearly identical for the samples possessing 1 and $5 \AA$ A-thick $\mathrm{Au}$ overlayers. This finding can be understood if $\mathrm{CO}$ only adsorbed on low-coordinated Au atoms, but not on the regular terraces (see results for $\mathrm{Au} / \mathrm{FeO}$ below), in good agreement with theoretical predictions (190). Therefore, it seems likely that gold deposition beyond $2 \AA$ thickness leads only to the gradual development of the flat terraces on the gold particles, which are inert towards $\mathrm{CO}$.

When examining the TPD data of CO adsorption on the Au/FeO samples as deposited at $77 \mathrm{~K}$, a strong particle size effect was seen, with desorption states observed at temperatures of up to almost $300 \mathrm{~K}$ for the lowest gold coverages (smallest particles) (302). After annealing to 500 $\mathrm{K}$, a reduction in the signal was observed and the size effect was completely suppressed, as TPD spectra showed two peaks at 130 and $200 \mathrm{~K}$, independent of the amount of gold deposited. This finding indicates that the particles after annealing have reached some minimum critical size beyond

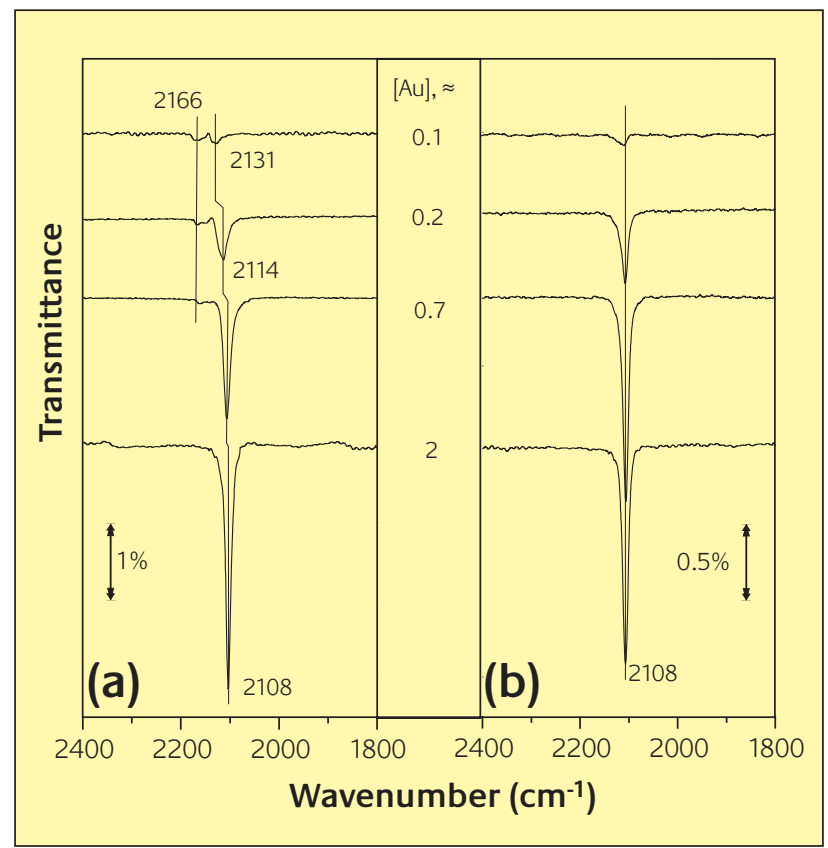

\section{Figure 20}

IRAS spectra of CO exposed to Au/FeO surface at $90 \mathrm{~K}$ as a function of Au coverage. (a) as deposited particles at $90 \mathrm{~K}$ (b) after annealing to $500 \mathrm{~K}$

which their adsorption behavior is size independent. However, particle size is not the only variable to consider as restructuring has likely taken place simultaneous to the sintering process and the low coordinated gold atoms which have been theoretically predicted to adsorb CO most strongly may have disappeared due to restructuring. In fact, we find that even monolayer islands formed after annealing to $500 \mathrm{~K}$, as seen in Fig.16, display the same CO adsorption behavior as large particles or gold single crystals (370). As the gold particles are known to nucleate on regular lattice sites as opposed to defects (302), we can exclude support effects. Therefore, we can conclude that size effects are only present in our system as such that smaller particles, particularly before annealing, contain a higher percentage of highly uncoordinated gold atoms which favor $\mathrm{CO}$ adsorption. In turn, we assert that quantum size effects related to particle thickness as proposed by Valden et al (192), are not likely to be a significant factor in $\mathrm{CO}$ adsorption.

Figure 20a shows IRAS spectra taken at saturated CO exposure for samples deposited at $90 \mathrm{~K}$ as a function of gold nominal thickness. At a coverage of $0.1 \AA$, a feature at 2166 $\mathrm{cm}^{-1}$ and a second at $2131 \mathrm{~cm}^{-1}$ are observed with approximately equal intensity. With increasing coverage, the higher frequency peak $\left(2166 \mathrm{~cm}^{-1}\right)$ decreases before disappearing at $2 \AA \mathrm{Au}$, while the second feature grows and shifts rapidly to lower wavenumbers from 2131 to $2108 \mathrm{~cm}^{-1}$ (371). This trend is different than that observed by previous researchers examining gold particles on thin alumina films, 
where CO adsorbed on the smallest particles was observed to be at lower numbers $\left(\sim 2100 \mathrm{~cm}^{-1}\right)$ and blue shifted to 2110 $\mathrm{cm}^{-1}$ as the particle size increased $(366,368)$. The origin of the difference between our system and these examples is unclear, but Goodman's group has recently studied $\mathrm{CO}$ adsorption on $\mathrm{Au}$ particles on $\mathrm{a} \mathrm{TiO}_{2}$ thin film and also observed adsorption frequencies higher than that of $\mathrm{CO}$ adsorbed on bulk gold, which they attributed to a support effect of unknown origin (372).

As shown in Fig. 20b, annealing to $500 \mathrm{~K}$ results the observation of a single state at $2108 \mathrm{~cm}^{-1}$ independent of the $\mathrm{Au}$ coverage with the disappearance of the state at $2165 \mathrm{~cm}^{-1}$, just as in the case of high gold coverage. Bearing in mind the sintering effects of annealing, the peak at $2108 \mathrm{~cm}^{-1}$ can be straightforwardly assigned to CO adsorbing on metallic gold. We propose that the species exhibiting the peak at $\sim 2165 \mathrm{~cm}^{-1}$ is probably CO adsorbed on very small clusters which can undergo charge transfer due to a specific interaction with the support initially on defects (371). Since at low coverages, the nucleation and growth is strongly influenced by steps and holes present on the FeO film (302), it seems likely that such charged species could be present. In previous IRAS studies on different catalysts systems like gold supported on zeolites (373), Au/ZrO2 (374), $\mathrm{Au} / \mathrm{TiO}_{2}$ (374-376) and $\mathrm{Au} / \mathrm{Fe}_{2} \mathrm{O}_{3}$ (377,378) similar CO stretching bands frequencies have been also detected and assigned to $\mathrm{CO}$ adsorbed on $\mathrm{Au}^{+}$species or Au interacting with hydroxyl groups on the surface (see Table 2). However, in our experiment, this feature appears after the main peak observed at $\sim 2108 \mathrm{~cm}^{-1}$ and therefore implies that CO adsorbs on such species more weakly relative to the metal clusters as has been seen by Fan et al (379), although this is in apparent contradiction with the view of some investigators that charged gold adsorbs CO more strongly (373,378,380-382). Interestingly, the single peak observed in the annealed samples did not shift and simply grew monotonically at increasing Au coverage, supporting our finding from TPD experiments indicating no apparent change in the possible adsorption states for $\mathrm{CO}$ on monolayer islands as compared to large particles. Therefore, we propose that CO adsorption on gold particles only includes the low coordinated surface atoms. This result can be traced back in order to explain saturation behavior for $\mathrm{CO}$ adsorption on gold deposited on alumina at the high coverages.

Gold supported by $\mathrm{Fe}_{3} \mathrm{O}_{4}$ is of particular interest due its proposed role in the water-gas shift cycle of $\mathrm{Au} / \mathrm{Fe}_{2} \mathrm{O}_{3}$ catalysts (11,383). It has been proposed that the dissociative adsorption of water on gold leads to a spillover of active hydroxyl groups onto the ferric oxide. The iron oxide surface is then reduced to $\mathrm{Fe}_{3} \mathrm{O}_{4}$ by the formation and decomposition of formate or carbonate intermediate species before subsequent reoxidation by dissociative adsorption of water. Furthermore, it has been shown by XPS that the surface of $\mathrm{Au} / \mathrm{Fe}_{2} \mathrm{O}_{3}$ catalysts can actually be dominated by $\mathrm{Fe}_{3} \mathrm{O}_{4}$ (384).

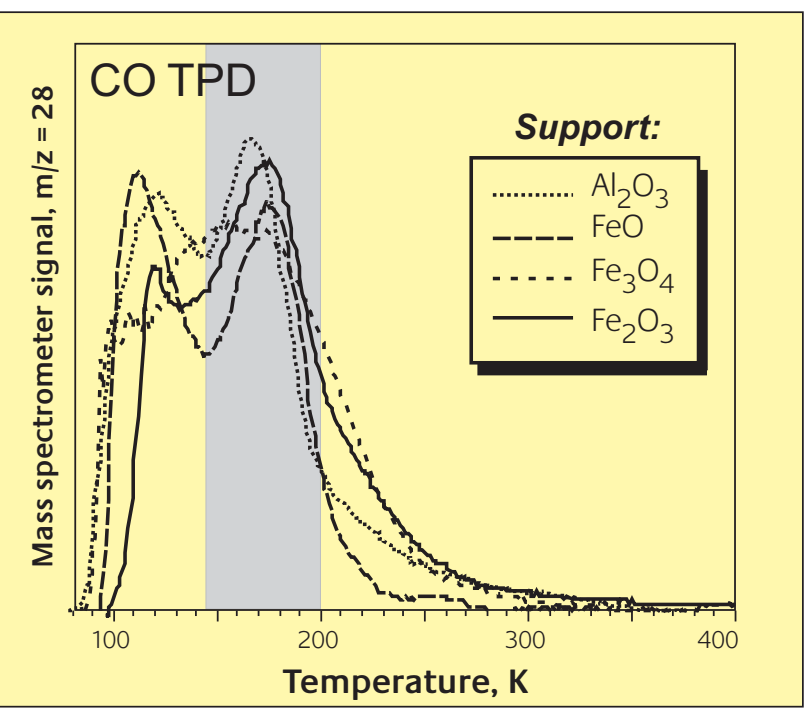

Figure 21

Comparison of CO TPD spectra for Au particles deposited on different oxide films showing that CO adsorption is essentially independent of the support at a given particle size $(\sim 3 \mathrm{~nm})$

The $\mathrm{CO}$ adsorption studies of $\mathrm{Au} / \mathrm{Fe}_{3} \mathrm{O}_{4}$ were more complicated relative to $\mathrm{FeO}$ and alumina films by the fact that the magnetite surface may adsorb $\mathrm{CO}$ and possesses desorption states which partly overlap with those of gold. After subtraction of the signals from the clean support, the CO TPD spectra showed the size effect as seen for other supports, i.e., the smaller the particles, the stronger the CO$\mathrm{Au}$ interaction (302). In contrast to the $\mathrm{FeO}(111)$ support, the effect was partly retained for $\mathrm{Au} / \mathrm{Fe}_{3} \mathrm{O}_{4}(111)$ even after heating to $500 \mathrm{~K}$. However, strong reduction of the CO desorption intensity clearly indicated the morphology changes upon annealing. For $\mathrm{CO}$ adsorbed on $\mathrm{Au} / \mathrm{Fe}_{2} \mathrm{O}_{3}$, the behavior is quite similar. The CO TPD spectra for "as deposited" at $90 \mathrm{~K}$ samples show a desorption state extended to $~ 300 \mathrm{~K}$, which disappear after annealing to $500 \mathrm{~K}$.

The CO adsorption studies on Au deposited on alumina and iron oxide films performed in our group clearly showed the particle size effect in that small particles adsorb CO more strongly. For the smallest Au coverage, CO may desorb at temperatures close to $300 \mathrm{~K}$, which is in the temperature range of the working Au catalysts for low temperature CO oxidation and has never been observed for Au single crystals. As discussed above, the presence of such a state is likely due to the presence of highly uncoordinated gold atoms.

In Fig. 21 CO TPD spectra obtained for gold deposited on various supports are compared for particles that have been annealed to $500 \mathrm{~K}$. The comparison clearly points out that, for a given particle size (ca. $3 \mathrm{~nm}$ as measured by STM), the interaction of CO with gold particles on different supports is essentially identical. Therefore, the results show that support effects frequently observed for CO oxidation on real catalytic 
Table 3

Results of CO adsorption on various gold surfaces.

\begin{tabular}{|c|c|c|c|c|c|}
\hline Surface & Particle size & Condition (CO pressure) & Frequency observed & Assignment & Ref. \\
\hline $\mathrm{Au} / \mathrm{SiO}_{2}$ & $5 \mathrm{~nm}$ & 0.3-84 torr @ 300 K & $\begin{array}{l}2120-2110 \\
2145\end{array}$ & $\begin{array}{l}\mathrm{Au}^{0} \\
\text { oxygen covered } \mathrm{Au}\end{array}$ & 542 \\
\hline Gold film & & $1 \mathrm{ML} @ 113 \mathrm{~K}$ & 2110 & & 81 \\
\hline $\mathrm{Au} / \mathrm{MgO}$ & $8 \mathrm{~nm}$ & 1-15 Torr @ 300 K & $2060-2040$ & multi-bonded CO & 444 \\
\hline $\mathrm{Au} / \mathrm{SiO}_{2}$ & $40 \mathrm{~nm}$ & 30 Torr @ $300 \mathrm{~K}$ & 2060 & & 444 \\
\hline Polycrystalline gold & - & 0.01-0.8 L@ @ K & $2125-2110$ & metallic gold & 82 \\
\hline $\mathrm{Au} / \mathrm{SiO}_{2}$ & & 10-3-700 torr @ $300 \mathrm{~K}$ & 2129-2106 & & 368 \\
\hline $\mathrm{Au}(332)$ & - & 0.5 \% ML CO-10 $0^{-5} \mathrm{mbar} @ 92 \mathrm{~K}$ & $2124-2110$ & metallic gold & 83 \\
\hline Au/Na-Y zeolite & & 0.5 torr @ 77K & $\begin{array}{l}2120 \\
2188 \\
2176\end{array}$ & $\begin{array}{l}\mathrm{Au}^{0} \\
\mathrm{Au}^{+} \\
\mathrm{CO} \text { condensed in zeolite pores }\end{array}$ & 450 \\
\hline $\mathrm{Au} / \mathrm{TiO}_{2}$ & & 38 torr @ $300 \mathrm{~K}$ & $\begin{array}{l}2113-2105 \\
2071-2038 \\
1670 \\
1580\end{array}$ & $\begin{array}{l}\mathrm{Au}^{0} \\
\text { bidentate carbonate } \\
\text { carboxylate }\end{array}$ & 403 \\
\hline \multirow[t]{2}{*}{$\mathrm{Au} / \mathrm{Al}_{2} \mathrm{O}_{3} / \mathrm{Mo}(110)$} & $0.5 \mathrm{ML}$ & $10^{-5} \mathrm{mbar} @ 100 \mathrm{~K}$ & 2100 & non-metallic gold & 367 \\
\hline & $20 \mathrm{ML}$ & & 2110 & metallic gold & \\
\hline Au/H-Y zeolite & $1-4 \mathrm{~nm}$ & 50 torr, $300 \mathrm{~K}$ & $\begin{array}{l}2111 \\
2138 \\
2169\end{array}$ & $\begin{array}{l}\mathrm{Au}^{0} \\
\mathrm{Au}^{+} \\
\mathrm{Au}^{+}\end{array}$ & 373 \\
\hline $\mathrm{Au} / \mathrm{Fe}_{2} \mathrm{O}_{3}$ & & 8 mbar CO, 8 mbar $\mathrm{O}_{2} @ 300 \mathrm{~K}$ & $\begin{array}{l}2116 \\
2138 \\
2159 \\
2340\end{array}$ & $\begin{array}{l}\mathrm{Au}^{0} \\
\mathrm{Au} \text { neighbouring } \mathrm{O} \\
\mathrm{Au}^{+} \\
\mathrm{CO}_{2}\end{array}$ & 378 \\
\hline $\mathrm{Au} / \mathrm{TiO}_{2}$ & $7.5 \mathrm{~nm}$ & 15 mbar CO, 15 mbar O $@ 300 \mathrm{~K}$ & $\begin{array}{l}2116 \\
2151 \\
2187 \\
2351\end{array}$ & $\begin{array}{l}\mathrm{Au}^{0} \\
\mathrm{Au}^{+} \\
\mathrm{Ti}^{4+} \\
\mathrm{CO}_{2}\end{array}$ & 376 \\
\hline $\mathrm{Au}_{8} / \mathrm{MgO} / \mathrm{Mo}(100)$ & $\sim 6 \AA$ & $90 \mathrm{~K}$ & 2102 & partially anionic $\mathrm{Au}$ & 247 \\
\hline $\mathrm{Au} / \mathrm{TiO}_{2}$ & & 2.66 kPa @ 298 K & $\begin{array}{l}2119 \\
2188 \\
2136\end{array}$ & $\begin{array}{l}\mathrm{Au}^{0} \\
\mathrm{Ti}^{4+} \\
\mathrm{Au} \text {, interacting with support }\end{array}$ & 375 \\
\hline $\mathrm{Au} / \mathrm{Fe}_{2} \mathrm{O}_{3}$ & $2.9 \mathrm{~nm}$ & $2.66 \mathrm{kPa} @ 298 \mathrm{~K}$ & $\begin{array}{l}2170 \\
2137\end{array}$ & $\begin{array}{l}\mathrm{Fe}^{3+} \\
\mathrm{Au} \text {, interacting with support }\end{array}$ & 377 \\
\hline $\mathrm{Au} / \mathrm{TiO}_{2}$ & $2 \mathrm{~nm}$ & $5 \mathrm{mbar} \mathrm{CO}$ in $1 \mathrm{~atm}$ of $\mathrm{He} @ 300 \mathrm{~K}$ & $\begin{array}{l}2115 \\
2180 \\
2350\end{array}$ & $\begin{array}{l}\mathrm{Au}^{0} \\
\mathrm{Ti}^{4+} \\
\mathrm{CO}_{2}\end{array}$ & 470 \\
\hline $\mathrm{Au} / \mathrm{Fe}_{2} \mathrm{O}_{3}$ & $\begin{array}{l}4.8 \mathrm{~nm} \\
11 \mathrm{~nm}\end{array}$ & 100 torr @ 300 K & $\begin{array}{l}2107 \\
2123\end{array}$ & & 422 \\
\hline $\mathrm{Au} / \mathrm{Fe}_{2} \mathrm{O}_{3}$ & $6.5 \mathrm{~nm}$ & 0.05-1.0 kPa @ $353 \mathrm{~K}$ & $2111-2104$ & $\mathrm{Au}^{0}$ & 22 \\
\hline $\mathrm{Au} / \mathrm{Na}-\mathrm{ZSM}-5$ & & 60 torr $\mathrm{CO}, 5$ torr $\mathrm{H}_{2} \mathrm{O} @ 323 \mathrm{~K}$ & $\begin{array}{l}2128 \\
2192 \\
1630\end{array}$ & $\begin{array}{l}\mathrm{Au}^{0} \\
\mathrm{Au}^{+} \\
\mathrm{HCOO} \text { on } \mathrm{Au}^{+}\end{array}$ & 543 \\
\hline $\mathrm{Au} / \mathrm{Al}_{2} \mathrm{O}_{3}$ & $4 \mathrm{~nm}$ & 266 Pa @150 K & $\begin{array}{l}2108 \\
2154 \\
2186\end{array}$ & $\begin{array}{l}\mathrm{Au}^{0} \\
\text { oxidized } \mathrm{Au} \\
\text { Lewis acid site } \mathrm{Al}_{2} \mathrm{O}_{3}\end{array}$ & 541 \\
\hline $\mathrm{Au}(110)$ & - & 0.1-100 torr @ $300 \mathrm{~K}$ & $\begin{array}{l}2110 \\
2141\end{array}$ & $\begin{array}{l}\text { Metallic gold } \\
\text { Physisorbed CO }\end{array}$ & 92 \\
\hline $\mathrm{Au} / \mathrm{ZrO} 2$ & $4.1 \mathrm{~nm}$ & 0.05-2.5 mbar @ $90 \mathrm{~K}$ & $\begin{array}{l}2116-2100 \\
2169 \\
2181 \\
2091 \\
2155\end{array}$ & $\begin{array}{l}\text { Au step on particle } \\
\text { support cations } \\
\text { support cations } \\
\text { Au particle edge } \\
\text { Au interacting with } \mathrm{OH} \text { groups }\end{array}$ & 374 \\
\hline $\mathrm{Au} / \mathrm{TiO} 2$ & $4.1 \mathrm{~nm}$ & 0.009-2.5 mbar@90 K & $\begin{array}{l}2109-2100 \\
2163 \\
2177 \\
2087 \\
2155\end{array}$ & $\begin{array}{l}\text { step on particle } \\
\text { support cations } \\
\mathrm{Ti}^{+{ }^{+}} \\
\text {Au particle edge } \\
\text { Au interacting with } \mathrm{OH} \text { groups }\end{array}$ & 374 \\
\hline $\mathrm{Au} / \mathrm{Al}_{2} \mathrm{O}_{3} / \mathrm{NiAl}(110)$ & $10-600$ atoms & 0.05-1.0 L @ $90 \mathrm{~K}$ & $2110-2098$ & & 369 \\
\hline $\mathrm{Au} / \mathrm{TiO}_{2}$ & $2-3 \mathrm{~nm}$ & $1 \mathrm{kPa} 02,1 \mathrm{kPa} \mathrm{CO}, 1 \mathrm{~atm} \mathrm{~N} 2 @ 80^{\circ} \mathrm{C}$ & $\begin{array}{l}2112 \\
2362 \\
2335 \\
1690,1586 \\
1440 \\
1400-1350\end{array}$ & $\begin{array}{l}\mathrm{Au}^{0} \\
\mathrm{CO}_{2} \\
\mathrm{CO}_{2} \\
\text { bidentate carbonate on } \mathrm{TiO}_{2} \\
\text { monodentate carbonate } \\
\text { formate }\end{array}$ & 400 \\
\hline $\mathrm{Au} / \mathrm{FeO}(111) / \mathrm{Pt}(111)$ & $\begin{array}{l}3 \mathrm{~nm} \times 3 \AA \\
7 \times 2 \mathrm{~nm}\end{array}$ & $0.1-1.5$ L@ $90 \mathrm{~K}$ & $\begin{array}{l}2108 \\
2131 \\
2165\end{array}$ & $\begin{array}{l}\text { Metallic gold } \\
\mathrm{Au}_{\text {clusters }} \\
\mathrm{Au}^{+}\end{array}$ & $\begin{array}{l}370, \\
371\end{array}$ \\
\hline $\mathrm{Au} / \mathrm{TiO}_{2} / \mathrm{Mo}(110)$ & $1.8-3.1 \mathrm{~nm}$ & $10^{-7}-10^{-2} \mathrm{mbar} @ 200 \mathrm{~K}$ & $2126-2118$ & $\mathrm{Au}^{0}$, interacting with support & 372 \\
\hline $\mathrm{Au}(110)$ & & $10^{-8}-10^{-4} \mathrm{mbar} @ 110-250 \mathrm{~K}$ & 2118-2108 & $\mathrm{Au}^{0}$ & 90 \\
\hline
\end{tabular}


systems may arise from the specific interaction of gold with oxygen rather than CO. However, the finding that annealing strongly attenuates size effects indicates that the nature of the support and its defect structure may be critical for nucleation, growth and stabilization of very small Au clusters upon which $\mathrm{CO}$ adsorbs more strongly. Just as we have observed slight differences between forms of iron oxide, Okumura et al had previously observed differences in the annealing behavior of gold on different forms of $\mathrm{TiO}_{2}$ and found that defect structure of the support strongly controlled the particle size distribution after calcination and therefore the reactivity (385).

Particle size effects have been observed in the adsorption of other molecules as well. Sykes et al observed that styrene was bound much more strongly to small gold particles on titania as compared to a thick gold film as the desorption temperature was shifted from $350 \mathrm{~K}$ to $410 \mathrm{~K}$ (278). Furthermore, the desorption signal from the titania surface itself at $250 \mathrm{~K}$ was lost when depositing only $0.25 \mathrm{ML}$ gold on the titania surface. This result implies that during the TPD experiment, styrene on the titania surface was mobilized and migrated to the gold particles where it then adsorbed in a much stronger manner.

It has been speculated that molecular oxygen could be adsorbed (and possibly dissociated) on small gold particles but to date no direct observation has been made. Zhu et al report the adsorption of molecular oxygen on gold particles on HOPG using XPS at room temperature following exposure of oxygen at $1 \mathrm{~atm}$ (386). However, as the particles are quite large $(20-50 \mathrm{~nm})$ it seems unlikely that they possess adsorption behavior greatly different from bulk gold, which has been conclusively shown to be inert to such exposures (95) and therefore it seems exceedingly unlikely that molecular oxygen could possibly be a stable species in UHV at $300 \mathrm{~K}$ on such structures.

Choi et al. showed desorption of oxygen from $\mathrm{Au} / \mathrm{SiO}_{2}$ following large (on the order of 10,000 L of gas) exposures of $\mathrm{NO}_{2}$ or $\mathrm{O}_{2}$ (387). Oxygen was observed to desorb in a peak at about $800 \mathrm{~K}$. The authors speculated that this peak arises from desorption of sub-surface oxygen. The amount of oxygen was shown to increase as the exposure temperature of the sample increased, indicating the chemisorption process is highly activated. The authors concluded that an interaction between the gold and the silica support was responsible for the increased chemisorption of oxygen on the supported gold surface (as opposed to gold single crystals).

In a similar fashion to the work on single crystals, the adsorption of atomic oxygen on gold particles on titania has been studied by Campbell and co-workers using a hot filament to dissociate oxygen (196). Campbell's group observed a very strong shift to higher oxygen desorption temperatures from $530 \mathrm{~K}$ to $645 \mathrm{~K}$ as the amount of gold

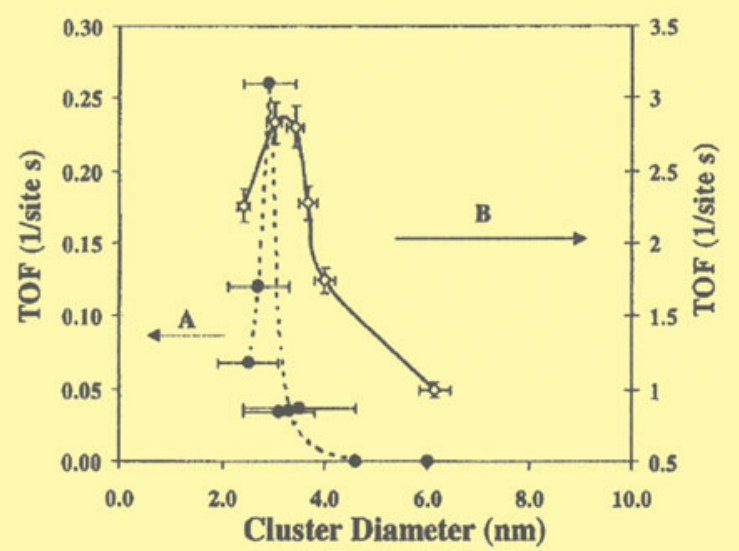

Figure 22

Turnover frequencies from (a) Au/TiO $/ \mathrm{Mo}(110)$ model catalysts grown in UHV (b) $\mathrm{Au} / \mathrm{TiO}_{2}$ catalysts prepared by deposition precipitation for $\mathrm{CO}$ oxidation at $300 \mathrm{~K}$ as a function of the Au particle size. 232 (data from 192, 395))

deposited was decreased from $6 \mathrm{ML}$ to $2.3 \mathrm{ML}$. For the thinnest islands, estimated to be a single monolayer, no oxygen desorption was observed below $710 \mathrm{~K}$ even though LEIS measurements of the attenuation of the gold signal confirmed the presence of oxygen on the surface. Due to their stronger binding of atomic oxygen, the authors suggested that gold particles below a critical thickness may have greatly enhanced oxygen dissociation capability. In addition, $\mathrm{CO}$ oxidation activity was demonstrated by the evolution of $\mathrm{CO}_{2}$ from the room temperature sample surface upon exposure of the oxygen covered $\mathrm{Au} / \mathrm{TiO}_{2}$ model catalyst to $10^{-6}$ torr of CO (388). TPD experiments showed total depletion of oxygen from the surface following CO exposure. Rate constant estimates based on the rate of disappearance of oxygen revealed that particles of two-layer thickness were approximately twice as reactive as one layer thick particles. As seen in the first experiments by Campbell's group, the thinner particles bind oxygen more strongly so it is not as easily available for reaction. At the same time, the thinner particles may have a diminished CO adsorption capacity. An apparent activation energy of $11.4 \mathrm{~kJ} / \mathrm{mol}$ was reported for the two-layer thick particles, which is not remarkably different from results reported by Outka and Madix on $\mathrm{Au}(110)$ or by Haruta for real catalysts.

Martra et al have recently observed that smoother gold particles on $\mathrm{SiO}_{2}$ were more reactive for $\mathrm{CO}$ oxidation although their CO adsorption capability was reduced (389). Like Bondzie et al (388), the authors theorized that oxygen was bound too strongly for reaction on particles with a higher number of low coordinated atoms.

The production of $\mathrm{CO}_{2}$ for temperatures as low as $65 \mathrm{~K}$ has been observed by Kim et al when impinging a molecular 
beam of $\mathrm{CO}$ on a $\mathrm{Au} / \mathrm{TiO}_{2}(110)$ sample that had previously been exposed to atomic oxygen (390). The authors reported that $\mathrm{CO}_{2}$ production is maximized for an oxygen coverage of about $0.1 \mathrm{ML}$. Although site blocking cannot be ruled out, the authors speculated that electronic effects were likely responsible for the reduction in $\mathrm{CO}$ adsorption and consequent loss of reactivity at higher oxygen coverages. Significantly, no particle size effect was observed, as the amount of $\mathrm{CO}_{2}$ increased with the amount of gold deposited (in the absence of gold no $\mathrm{CO}_{2}$ is produced). This result combined with findings from the Campbell group (388) suggests that mechanisms involving atomic oxygen are only slightly particle size dependent. Furthermore, the tendency of larger particles to be more reactive is counter to observations made when the oxygen is not atomically dosed and seems to imply that oxygen dissociation is the ratelimiting step. Mullins' group also reported that for temperatures above the desorption temperature of $\mathrm{CO}$, the reaction rate decreased rapidly following exposure to $\mathrm{CO}$, but then rose slightly showing a second maximum in production, frequently several seconds later (391). The authors have speculated that two different mechanisms (possibly one E-R mechanism and one $\mathrm{L}-\mathrm{H}$ mechanism) must be present in order to explain the presence of the second maximum.

Recently, Mullins' group has found evidence for a molecular oxygen species on $\mathrm{Au} / \mathrm{TiO}_{2}$ model catalysts. Some molecular oxygen was found to be delivered to the surface as a by-product of the oxygen atom source used in the experiments described above (392), and is likely in some electronically excited state. The reactivity of the molecular oxygen species as compared to atomic oxygen is the subject of further research (393).

Goodman's group $(192,394)$ made use of a high pressure cell (40 torr) and were able to observe $\mathrm{CO}_{2}$ production by a planar $\mathrm{Au} / \mathrm{TiO}_{2}$ catalyst when exposing the surface to a 1:5 $\mathrm{CO}: \mathrm{O}_{2}$ mixture at room temperature. They observed a strong particle size effect with those particles in the range of 2.5$3.5 \mathrm{~nm}$ showing a maximum in activity. Although Haruta originally observed that the activity increases continuously as the particle size decreases $(9,187,343)$, a careful examination of the data presented by Bamwenda et al (395) reveals extraordinary agreement with the findings of the Goodman group (192,394). As discussed above, Valden et al related this maximum in activity to the transition between metallic and non-metallic particles. Variations in particle size also gave variations in the apparent activation energy (3.5-6.0 $\mathrm{kcal} / \mathrm{mol})$. The authors concluded that this must result from an intrinsic property of the particles and not from any geometric effect due to the changing ratio of perimeter atoms to surface atoms. Gold particles with an average diameter of $2.4 \mathrm{~nm}$ were shown to lose activity rapidly over the first hour of exposure to the gas mixture at room

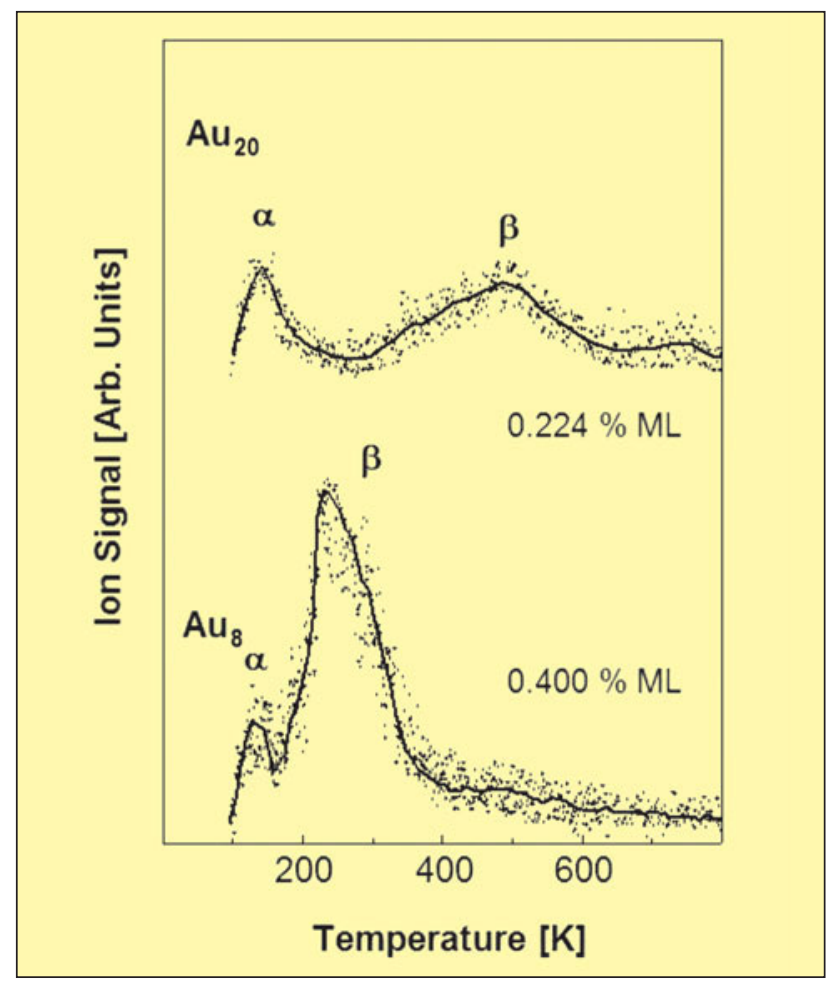

\section{Figure 23}

Temperature programmed reaction spectra for $\mathrm{CO}_{2}$ production following $\mathrm{CO}$ and $\mathrm{O}_{2}$ exposure at $90 \mathrm{~K}$ to $\mathrm{Au}_{8}$ and $\mathrm{Au}_{20}$ clusters on $\mathrm{MgO}$. (405)

temperature and AES showed an increase in carbon on the surface. Efforts to regenerate the catalyst with oxygen were not successful in spite of the removal of carbon.

Valden et al (192,394) and work by Haruta (9, 191, 395, 396) report a strong size effect: particles above $5 \mathrm{~nm}$ exhibit virtually no activity while particles below $5 \mathrm{~nm}$ show good activity for room temperature $\mathrm{CO}$ oxidation, exhibiting a maximum in activity at 2.8-3.2 nm as shown in Figure 22. The observation of such size effects in catalysis is not new (397,398). However, the difference in activity between bulk gold and supported gold particles is rather dramatic. In the widely studied oxidation of $\mathrm{CO}$, oxide supported catalysts have been shown to be at least two orders of magnitude more active than unsupported gold (399). However, how and why supported gold particles experience an increased reactivity is still largely unknown.

However, it appears likely that the exact size range which gives a maximum in activity may depend upon the reaction or the support or even the catalyst preparation method. For example, Schumacher et al. have recently reported that the maximum in activity below $2 \mathrm{~nm}$ for their $\mathrm{Au} / \mathrm{TiO}_{2}$ catalyst (400). Prati and co-workers have found for the oxidation of ethylene glycol to glycolate that the activity increased with decreasing particle size for $\mathrm{Au} / \mathrm{Al}_{2} \mathrm{O}_{3}$ and $\mathrm{Au} / \mathrm{TiO}_{2}$ catalysts but that the rate exhibited a maximum at some intermediate size for activated carbon supported gold catalysts $(401,402)$. 


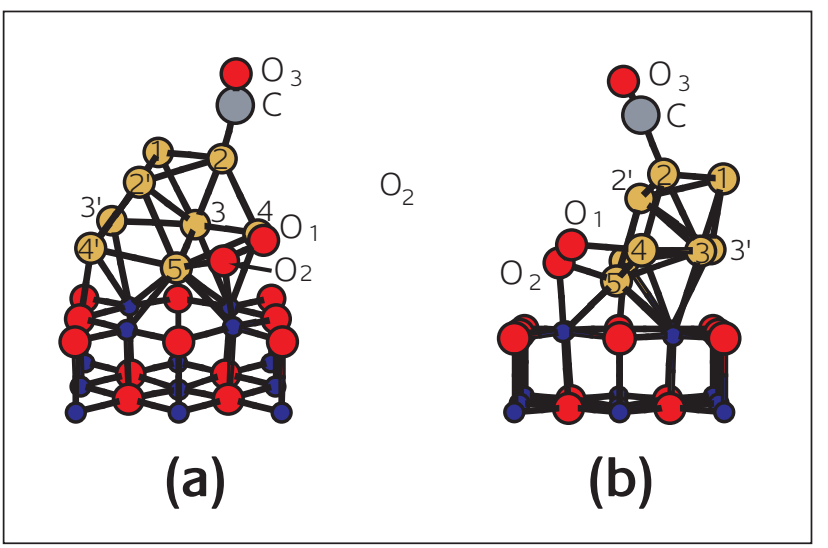

Figure 24

(a) Front and (b) side views of the CO oxidation reaction on an $\mathrm{Au}_{8}$ cluster. CO is conceived to adsorb on-top of the cluster while oxygen is conceived to adsorb molecularly at the particle periphery. (247)

Some authors have even reported that catalytic activity and particle size had little or no relationship (403). The picture is often muddled by the lack of a monodisperse size distribution. Heiz and co-workers have attempted to address this problem by using a mass spectrometry size selection technique, by which clusters from 1-20 gold atoms in size were examined on an $\mathrm{MgO}$ thin film substrate (247,404-406). Sanchez et al found that the onset of $\mathrm{CO}$ oxidation activity occurred at 8 atoms, below which no activity was observed and above which catalytic activity increased in an oscillatory fashion. The $\mathrm{CO}_{2}$ desorption temperature was also found to be cluster size dependent. As shown in Figure 23, for $\mathrm{Au}_{8}, \mathrm{CO}_{2}$ was produced in two peaks, a low temperature feature at $140 \mathrm{~K}$ and a larger feature at $240 \mathrm{~K}$. As the cluster size increased, the desorption temperature of the high temperature feature also increased (up to $500 \mathrm{~K}$ for $\mathrm{Au}_{20}$ ) while the low temperature feature remained constant. It is notable that CO was observed to desorb between 150 and 180 $\mathrm{K}$ from the $\mathrm{Au}_{8}$ clusters in the absence of oxygen. Therefore, as has been seen in gas phase experiments, the adsorption of $\mathrm{CO}$ and $\mathrm{O}_{2}$ must be cooperative such that $\mathrm{CO}$ (or at least some kind of transition state) is stabilized to much higher temperatures in the presence of oxygen. Similarly, oxygen may be stabilized by the presence of $\mathrm{CO}$, although at this point the adsorption/desorption behavior of oxygen on $\mathrm{Au}_{8}$ remains a mystery. Supporting calculations indicated that although the dissociation of oxygen is thermodynamically favored, the large barrier to reaction implied that the reaction should proceed through molecular oxygen.

Calculations also showed that while the adsorption of oxygen was highly dependent upon the cluster size, the adsorption of $\mathrm{CO}$ is relatively cluster size insensitive. The reaction was conceived to occur with the adsorption of CO on the cluster and the adsorption of oxygen on the particle periphery as shown in Figure 24. $\mathrm{CO}_{2}$ production was greatly enhanced for Au clusters on defect rich films as compared to

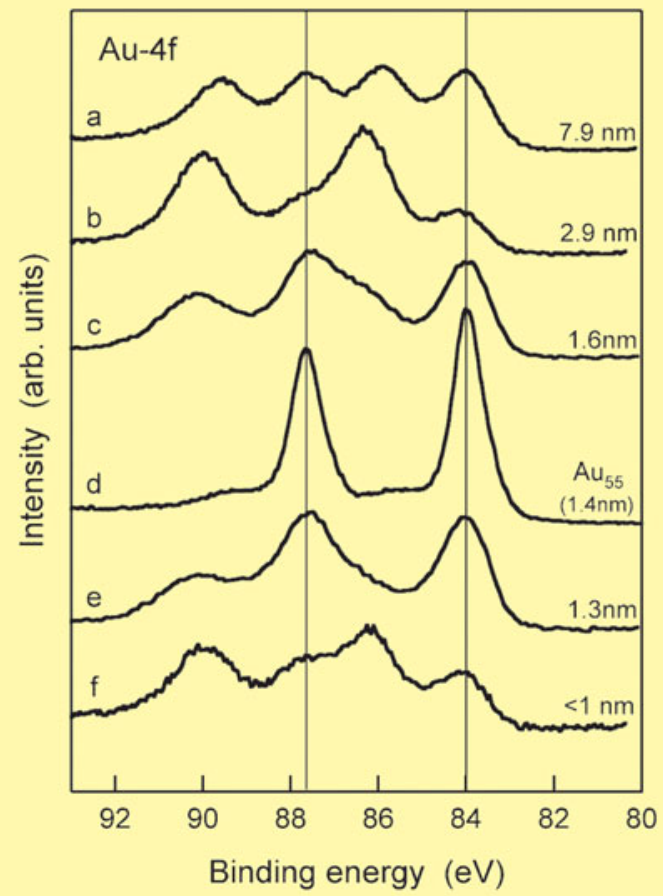

Figure 25

The Au 4 f region of XPS spectra obtained for a Au $u_{55}$ clusters deposited on a silica wafer and Au particles formed by metal evaporation on the same support following oxygen plasma treatment. The results show a high oxidation resistance of the closed-shell Aus5 cluster. (418)

clusters on defect poor films. Likewise, simulations indicated that the reaction barrier of $0.8 \mathrm{eV}$ was lowered to $0.5 \mathrm{eV}$ on $\mathrm{Au}_{8}$ clusters above defects due to the $0.5 \mathrm{eV}$ charge that is predicted to spread over the 8 atom cluster (247). Interestingly, $\mathrm{Pd}_{8}$ clusters did not exhibit the same defect dependent reactivity (405). Doping the clusters with one strontium atom was found to increase the reactivity of the clusters and clusters as small as $\mathrm{Au}_{3} \mathrm{Sr}$ showed appreciable activity (407). Perhaps surprisingly, the CO desorption temperature was not greatly different from that observed by Hollins and Ruggerio for $\mathrm{CO}$ on $\mathrm{Au}(332)$ (83). Moreover, IR data reveal a single peak at $2102 \mathrm{~cm}^{-1}$, for the C-O stretching frequency, which is in agreement with other data for $\mathrm{CO}$ on neutral bulk gold surfaces (see Table 2). This would seem to indicate an absence of special binding sites connected to anionic particles and the origin of the enhancement of CO adsorption on $\mathrm{Au}_{8}$ clusters remains unclear. For the $\mathrm{Au}_{8}$ clusters, the oxygen exposure-CO exposure- TPR cycle (to 350 $\mathrm{K})$ could be repeated several times without observing any drop off in $\mathrm{CO}_{2}$ production. This stability implies that the dispersed clusters were strongly enough bound to the $\mathrm{MgO}$ defects that they did not sinter.

Both Goodman's group (408) and Gates' group (409) have succeeded in preparing $\mathrm{Au}_{6}$ clusters using more conventional catalyst preparations. Goodman and co-workers employed a 
$\mathrm{Au}_{6}\left(\mathrm{PPh}_{3}\right)_{6}\left(\mathrm{BF}_{4}\right)_{2}$ precursor on a $\mathrm{TiO}_{2}(110)$ support which after electron stimulated desorption of the ligands gave highly dispersed $\mathrm{Au}_{6}$ clusters as seen by STM (408). In order to avoid problems with phosphorus contamination, Gates and Guzman opted to use $\mathrm{Au}\left(\mathrm{CH}_{3}\right)_{2}\left(\mathrm{C}_{5} \mathrm{H}_{7} \mathrm{O}_{2}\right)$ on $\mathrm{MgO}$ powder (409). After holding the catalyst in a He atmosphere at 373 $\mathrm{K}$ for 2 hours, EXAFS spectra indicated the formation of $\mathrm{Au}_{6}$ clusters. However, the reactivity of these novel systems has not been established.

Based on the above results and results from gas phase experiments, some researchers have advanced the idea of "magic number" clusters (410) that exhibit extraordinary activity. From their combined analysis of activity data, TEM and Debye function analysis (DFA) of the XRD spectra, Cunningham et al proposed that icosahedral $A_{13}$ particles on a $\mathrm{Mg}(\mathrm{OH})_{2}$ support acted as the active species $(411,412)$. The 13 atom clusters were conceived to be essentially constructed entirely from 5-fold coordinated atoms. Calcination at higher temperatures led to agglomeration and thereby less active catalysts, while calcination at lower temperatures left oxidic gold oligomers on the surface that failed to show appreciable activity. Examination of a used catalyst showed a complete absence of icosahedral structures and truncated decahedra had formed instead. In a comparison of supports, however, DFA identified fcc cubooctahedral gold structures from 1-8 $\mathrm{nm}$ to be the catalytically active species on $\mathrm{TiO}_{2}$ (413). For example, $\mathrm{Au}_{147}$ cubooctahedral particles formed the dominant species for a $1.3 \mathrm{wt} \% \mathrm{Au} / \mathrm{TiO}_{2}$ catalyst. Therefore, Cunningham and co-workers concluded that the catalytically active structures are dependent on the support and must be considered on a case-by-case basis.

Cleveland et al found in their examination of gold particles grown in solution that certain size clusters were preferentially formed as determined by XRD (210). The "magic sizes" of 75, 101, 146 atom clusters were found to be remarkably stable as XRD patterns of the same samples stored in air taken one year later were identical.

One of the first examples of a magic cluster is $\mathrm{Au}_{55}$ which was discovered by Schmid et al (414) and has since seen extensive study (415-417). Boyen et al recently confirmed that $\mathrm{Au}_{55}$ clusters display unusual chemical behaviour (418). They deposited two types of gold nanoparticles on a silicon wafer, $\mathrm{Au}_{55}$ Clusters and non-size selected particles ranging in average size from $1.0 \mathrm{~nm}$ to $8.0 \mathrm{~nm}$. XPS measurements were used to determine the degree of oxidation after exposure to an oxygen plasma. For the larger particles, the XPS spectra revealed four peaks for the Au $4 f$ region, including two associated with $\mathrm{Au}_{2} \mathrm{O}_{3}$ produced as a result of the plasma and two associated with metallic gold in the particle interior. Remarkably, the particles in the region around 1.6-1.3 nm did not show significant oxidation and the Aus5 clusters (with $d=1.4 \mathrm{~nm}$ ) showed no oxidation as shown in Figure 25. However, particles under $1 \mathrm{~nm}$ exhibited significant oxidation, seemingly indicating a maximum in oxidation resistance for the $\mathrm{Au}_{55}$ clusters. The valence band region spectra showed that the $\mathrm{Au}_{55}$ clusters showed small intensity right up to the Fermi level as is seen for bulk gold. Therefore the authors concluded that the remarkable resistance to oxygen was not a consequence of electron confinement but rather due to an electronic closed shell configuration.

\section{Connection to real catalysts}

\subsection{What is the active site?}

Currently, the real nature of gold catalysis, particularly with regard to the well-studied low temperature co oxidation reaction, remains a subject of much debate. In general, as depicted in Figure 26, CO is envisioned to adsorb on the gold particles themselves, probably on edge or step sites $(3,4)$. The adsorption site of oxygen is less clear, although mechanisms involving oxygen adsorption on the support or at the particle-support interface are often invoked as will be discussed in Section 6.2. The following ideas or combinations thereof have been proposed by researchers to explain the phenomenal reactivity of gold nanoparticles:

1 Small gold particles possess unique adsorption sites that allow for such reactions as the adsorption or dissociation of oxygen due to the presence of highly uncoordinated atoms, $(190,197,221,222,369,419,435,436)$.

2 Small gold particles have unique electronic and thereby chemical properties as a consequence of the quantum size effect. Some have amended this idea with the corollary that the thickness of the gold particle is the critical parameter. The role of the support is merely to stabilize these small particles $(192,196,323,388,392)$.

3 The active site for gold catalysis involves $\mathrm{Au}^{+}$whose presence may result from the catalyst precursor or the calcination treatment $(378,444-458,460)$.

4 The active site for gold catalysis is an anionic gold (Au) species which results as a consequence of the interaction of a particle with a $\mathrm{F}$ center defect in the underlying oxide support and which allows for the adsorption (and possible dissociation) of oxygen as shown in gas phase experiments $(170,184,185,220,247,374,462-465)$.

5 The active site for catalysis involves the interface between the particle and support. For CO oxidation it is generally proposed that $\mathrm{CO}$ adsorbs on the surface of the gold particle whereas oxygen adsorbs on the metal oxide support and moves to the metal/support interface or adsorbs there directly $(226,365,403,470-472,474,475)$.

We have previously discussed size effects in gold catalysis, which arise either from electronic effects, structural effects or a combination thereof. In spite of the data found 


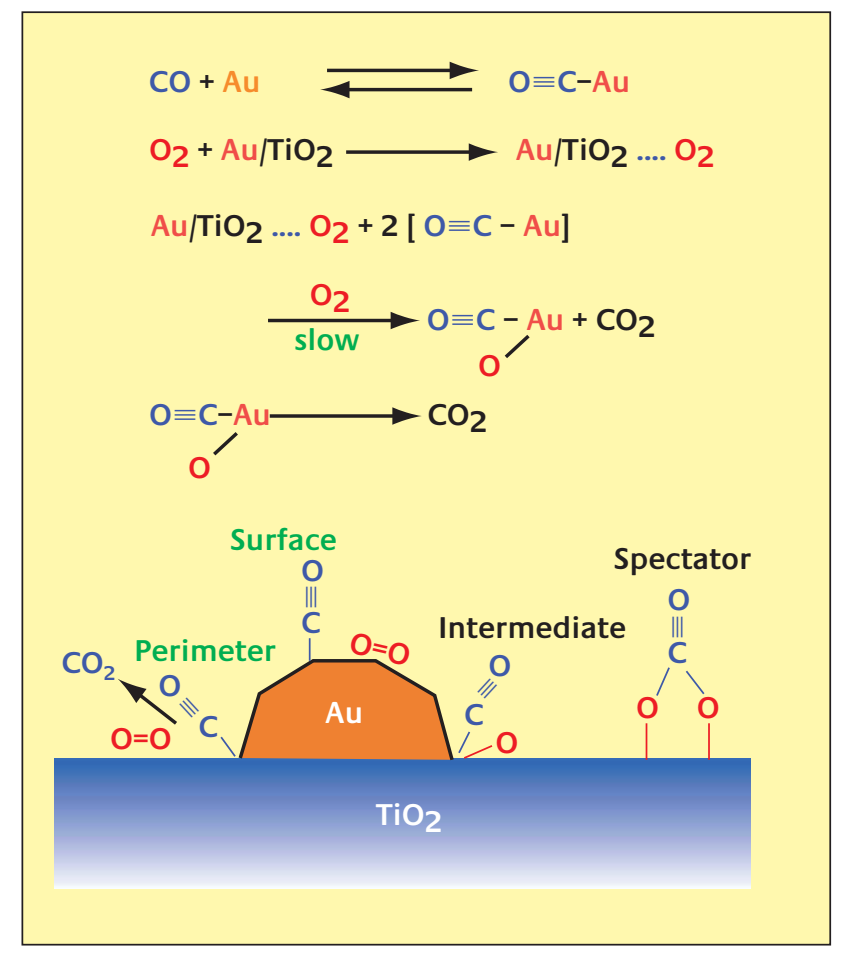

Figure 26

Schematic representation for CO oxidation pathways over Au/TiOz. (3)

in both the catalytic and surface science communities, particularly the dramatic results from size selected clusters, many experiments involving real catalysts report that size effects are not a critical factor in determining activity $(365,403,419,420)$. We are therefore left to question what the controlling factors for $\mathrm{CO}$ oxidation really are.

Recently Haruta has established a group of conditions whereby active gold catalysts can be made $(3,4)$. First and foremost Haruta asserts strong contact is necessary between gold and the underlying support. Although calcinations at high temperatures generally leads to agglomeration of particles which is assumed to be undesirable (395,421-423), Haruta and co-workers found an increase in activity with increasing calcination temperature between mechanically mixed $\mathrm{Au}$ colloid/ $/ \mathrm{TiO}_{2}$ powder samples calcined at $573 \mathrm{~K}$ and $873 \mathrm{~K}$ (424). TEM results showed the more active catalyst, in spite of its larger particle size, had better contact with the support. As a second point, Haruta argues that the proper support must be chosen. Basic supports are preferred for the type of deposition precipitation method that has been shown to be effective for the preparation of active catalysts. Traditional impregnation methods with gold chloride are unsatisfactory because during calcination of the catalyst, the gold particles experience severe agglomeration as $\mathrm{HAuCl}_{4}$ interacts only weakly with the support. The deposition precipitation method favored by Haruta (although many other methods have achieved at least limited success as well) relies upon an exchange between gold chloride and $\mathrm{NaOH}$ such that $\mathrm{Au}(\mathrm{OH})_{3}$ is formed and precipitates on the support. The dispersion and size of the particles, and therefore their catalytic activity, depend critically upon the pH used (typically 6-10) and the amount of gold in solution (423,425-432). After washing to remove $\mathrm{Cl}$ and $\mathrm{Na}$ ions, the catalyst is dried and calcined. In contrast to $\mathrm{HAuCl}_{4}$, $\mathrm{AuOH}_{3}$ agglomerates only minimally under calcining conditions. However, with the deposition precipitation method supports such as activated carbon (433) and zeolites (434) cannot be used due to their high acidity (isoelectric point). (It is interesting to point out that graphite substrates have often been used in photoemission experiments designed to explore the electronic properties of gold nanoparticles and that such particles appear to share the similar electronic character to gold particles on oxides). Finally, as a third condition, particles should be in a particular size range, although under the right circumstances particles as large as $10 \mathrm{~nm}$ can exhibit high activity. However, in the case of real catalysts, size distributions tend to be rather wide and difficulties may be encountered in the detection of very small particles, which could be the active species.

The effect of geometry has been discussed from a theoretical standpoint, but it is important to mention that some recent experimental results from real catalytic systems have also emphasized the influence of geometrical effects. As mentioned earlier Baiker's group found that the activity of $\mathrm{Au} / \mathrm{TiO}_{2}$ was much higher than $\mathrm{Au} / \mathrm{ZrO}_{2}$ found catalysts with the same particle size 359,419. Originally ascribed to support effects, HREM revealed that the differences in activity may arise from differences on particle shape. $\mathrm{Au} / \mathrm{TiO}_{2}$ catalysts had more highly uncoordinated atoms and were thereby more active as has been predicted by Nørskov and coworkers 190,222.

Mohr et al have identified reaction sites on the corners of gold particles for hydrogenation of acrolein to allyl alcohol $(435,436)$. In a comparison of $\mathrm{Au} / \mathrm{TiO}_{2}$ and $\mathrm{Au} / \mathrm{ZrO}_{2}$, Mohr et al attributed differences in reactivity to the degree of rounding of the particles. Although, particles on the two supports were nearly the same size, $\mathrm{Au} / \mathrm{TiO}_{2}$ was approximately twice as active and more selective as well for the production of allyl alcohol as $\mathrm{Au} / \mathrm{ZrO}{ }_{2}$. From an analysis of TEM images, the authors attributed these differences in reactivity to the presence of more highly uncoordinated atoms on particles supported by titania. More recently, by HREM it was found that indium wets the flat surfaces on gold leaving only the edges exposed (436) as shown in Figure 27. The authors found that zinc oxide supported $10 \mathrm{~nm}$ gold particles covered with indium were still half as reactive as those not covered by indium with a much better selectivity to allyl alcohol. This led the authors to conclude that the exposed corners and edges must be the reactive site for the selective hydrogenation of the $\mathrm{C}=\mathrm{O}$ group as $\mathrm{ZnO}$ itself is not active for the reaction. 
As mentioned above, Campbell (196) and Goodman (192) have suggested that two-dimensional islands of gold may exhibit unusual activity as compared to their 3-D counterparts. However, in our own work we failed to see any differences in $\mathrm{CO}$ adsorption between gold monolayers on FeO as compared to larger 3-D particles (370). Boccuzzi et al has previously suggested that 2-D rafts of gold may easily absorb negative charge from the support based on their observation of bands around $2050 \mathrm{~cm}^{-1}$ for $\mathrm{CO}$ adsorption on reduced $\mathrm{Au} / \mathrm{TiO}_{2}$ (437). As mentioned previously, Blick et al observed the presence of two-dimensional particles of gold by TEM and Mössbauer spectroscopy. The authors found that for the oxidation of methane, 2-D rafts were essentially inactive and served only to block methane coupling reactions that probably occur on oxygen vacancies now covered by gold (299). The authors proposed that 3-D particles of gold were necessary for reaction and that the reaction took place at the particle support interface. Although the presence of $\mathrm{Au}^{-}$sites associated with two-dimensional gold rafts was observed by $\mathrm{CO}$ adsorption on both $\mathrm{Au} / \mathrm{TiO}_{2}$ and $\mathrm{Au} / \mathrm{Fe}_{2} \mathrm{O}_{3}$, Boccuzzi et al found that the reverse water gas-shift reaction were only catalyzed by three-dimensional gold particles as the resulting $\mathrm{CO}$ formed was found only on $\mathrm{Au}^{0}$ sites (438).

Early EXAFS and XPS results detected the presence of oxidic gold in active catalysts (439-443). Lee and Schwank were among the first to suggest that such cationic species acted as the active sites in gold catalysis (444). Based on IR data which showed CO adsorption $2040 \mathrm{~cm}^{-1}$, considered to be on sites other than metallic gold, and considering the lack

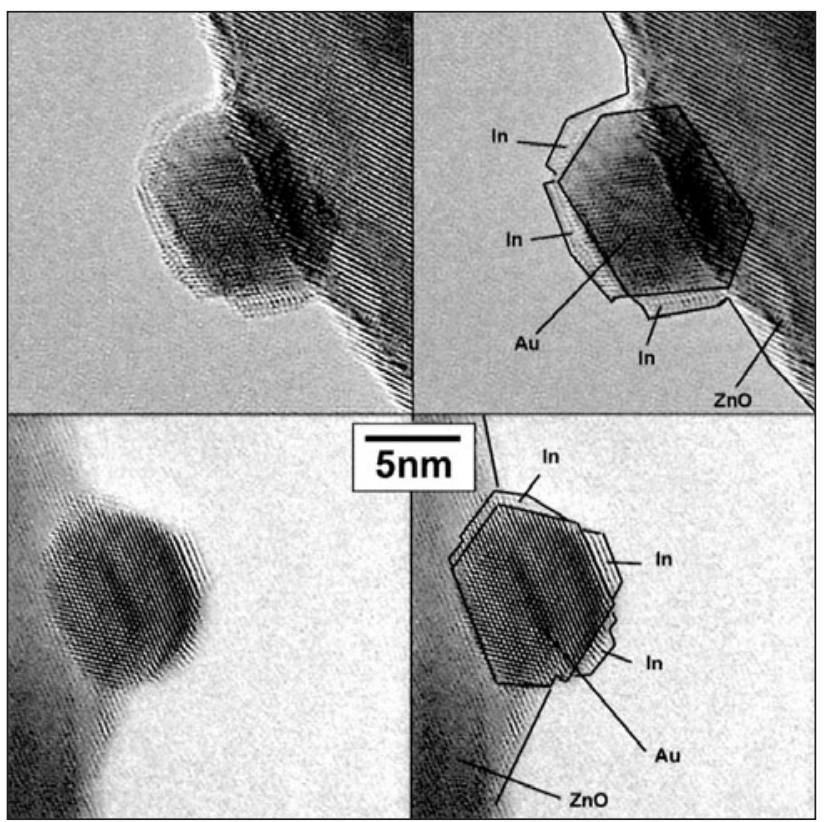

Figure 27

High resolution TEM images of two different indium covered Au particles (left side) on ZnO with corresponding assignment of structures (right). (436) of activity for unsupported bulk gold, the authors proposed that these adsorption sites may be related to gold that is electronically modified by the support due to charge transfer. More recently, infrared spectroscopy of CO adsorption on gold catalysts has revealed features between $2150-2160 \mathrm{~cm}^{-1}$ (as seen in Table 2) which has led some researchers to invoke a mechanism which involves $\mathrm{Au}^{+}(378,382,445)$. XPS and FTIR experiments by Galvagno and co-workers have shown a loss of $\mathrm{Au}^{+}$species over time when $\mathrm{Au} / \mathrm{Fe}_{2} \mathrm{O}_{3}$ catalysts were exposed to reactive mixtures $(378,446)$. The authors concluded that the deactivation of the catalyst over time was directly related to the loss of $\mathrm{Au}^{+}$and the growth of $\mathrm{Au}^{0}$. In most studies, an increase in the calcination temperature resulted in a decrease in the catalyst activity. Park and Lee tied this effect to the presence or absence of oxidic gold (447). As calcination temperature of $\mathrm{Au} / \mathrm{TiO} 2$ was increased from $373 \mathrm{~K}$ to $673 \mathrm{~K}$, the activity was observed to fall by a factor of 10. At the same time, XPS and XANES data showed a transition from an oxidic gold species similar to $\mathrm{Au}(\mathrm{OH})_{3}$ to a metallic gold species whose photoemission spectra were indistinguishable from a gold foil. Hao and co-workers later confirmed this result for $\mathrm{Au} / \mathrm{Fe}_{2} \mathrm{O}_{3}$ catalysts (448). Hao et al speculated that the calcinations process transforms $\mathrm{Au}^{3+}$ to $\mathrm{Au}^{\mathrm{d}+}$ essentially giving $\mathrm{Au}$ a similar electronic structure to Pt. In similar work using Mössbauer spectroscopy, Kobayashi et al found that the most active $\mathrm{Au} / \mathrm{Mg}(\mathrm{OH})_{2}$ catalysts also had the largest amount of $\mathrm{Au}^{1+}(449)$ and thereby concluded that oxidic gold is the active species for CO oxidation. Ichikawa and co-workers also asserted that $\mathrm{Au}^{+}$is the active component based on their finding that $\mathrm{Au}^{1+} / \mathrm{NaY}$ zeolite catalysts were considerably more active than their $\mathrm{Au}^{0}$ counterparts (450) for reduction of NO. The authors proposed that positively charged gold species acts as a good catalyst due to its unfilled $d$-band (451). The active site was conceived to be $\mathrm{Au}^{1+}$ ions that exist at the interface between metallic gold particles and the zeolite support (450). Furthermore it was found that $\mathrm{Au}^{1+}$ catalysts were more active than $\mathrm{Au}^{3+}$ (452).

Unlike the findings from Ichikawa's group, other investigations have found that higher oxidation states were even better catalysts. Waters et al saw, in their comparison of Au on various supports, that the activity for methane oxidation increased directly with the oxidation state of gold as observed by XPS (453). The authors hypothesized that oxidized gold had a greater tendency to chemisorb oxygen. Gates and Guzman recently determined that $\mathrm{Au}^{3+}$ is the active species for hydrogenation of ethylene by gold (454). XANES data revealed a direct correlation between the coordination number and the activity of $\mathrm{MgO}$ supported gold catalysts. An increase in activity was also observed with increasing amounts of $\mathrm{Au}^{3+}$ and the best catalyst was found to be a mononuclear gold complex formed by deposition of 
$\mathrm{Au}^{3+}\left(\mathrm{CH}_{3}\right)_{2}\left(\mathrm{C}_{5} \mathrm{H}_{7} \mathrm{O}_{2}\right)$. Similar to Ichikawa's group, Wan and coworkers ascribed deactivation of their Au/Y zeolite catalysts to transformation of $\mathrm{Au}^{3+}$ to $\mathrm{Au}^{0}$ as seen by XPS (455).

Hutchings and co-workers found that uncalcined iron oxide supported gold catalysts were considered more active than those calcined at $400^{\circ} \mathrm{C}(456,457)$. They attributed this large difference in reactivity to the presence of goldoxyhydroxide (AuOOH. $\mathrm{H}_{2} \mathrm{O}$ ) as observed by $\mathrm{Au}^{197}$ Mössbauer spectroscopy. Unlike many other studies, the activity of their catalyst increased over time. This could be an indication that further changes to the catalyst (such as partial reduction by $\mathrm{CO}$ ) are beneficial to the catalyst's performance. TEM examination showed that while the relatively inactive calcined catalysts showed particles in the 3-5 nm range, which are generally thought to be of ideal size, the more active dried samples revealed a more homogeneous distribution of gold. Kang and Wan found that iron modified Au/Y zeolite catalysts could be regenerated with water vapor (458). Based on this result, they proposed that gold hydroxide in intimate contact with the oxide support acted as the active species in CO oxidation.

Some have even proposed that gold is essentially incorporated into the support itself $(365,455,459)$. If gold possesses the same valence as the support cation, then it is reasonable to suggest that gold could simply replace the support metal. Sze et al found evidence of this behavior using Mössbauer spectroscopy for $\mathrm{Au} / \mathrm{Fe}_{2} \mathrm{O}_{3}$ catalysts (459). Using a co-precipitation preparation method it was possible to insert gold into the iron oxide lattice. However, upon annealing above $300^{\circ} \mathrm{C}$, gold was once again segregated from the $\mathrm{Fe}_{2} \mathrm{O}_{3}$ phase. Wan and Kang had similarly proposed that gold was incorporated into the lattice of a $Y$ zeolite during preparation and that in fact the point at which this substitution occurred then acted as a nucleation site for further gold deposition (455).

Flytzani-Stephanopoulos and co-workers have recently demonstrated that gold nanoparticles are not the active species for the water-gas shift reaction seen on $\mathrm{Au} / \mathrm{CeO}_{2}$ catalysts (460). The authors found that using a leaching process almost all the metallic gold could be removed from $\mathrm{Au} / \mathrm{CeO}_{2}$ that had been prepared by deposition precipitation or co-precipitation, leaving behind a highly dispersed gold species. These leached catalysts exhibited comparable activity to the as prepared catalysts leading the authors to conclude that metallic gold must not contribute to the catalyst's reactivity. Flytzani-Stephanopoulos and co-workers support a model in which $\mathrm{Au}$ is integrated into the ceria support. The authors suggest that the role of $\mathrm{Au}$ is to increase the amount of surface oxygen in ceria (461).

In contrast to the situation with cationic gold, relatively less attention has been focused on the presence of anionic gold as the active species. However, as mentioned above, oxygen vacancies on titania and magnesia are preferable nucleation sites for gold (246,251-255). In addition, Heiz and co-workers have demonstrated that gold above such defects will exhibit higher activity (247). Calculations indicate that gold above the oxygen vacancy is electron rich as a charge transfer is imagined to take place from the support to the gold particle. Such an active site would also be consistent with gas phase experiments which demonstrated that oxygen preferred to adsorb on negatively charged clusters (170). Since there is some evidence that oxygen adsorption (and dissociation) is the rate-limiting step $(87,196,388)$, the presence of anionic clusters is perhaps crucial to the reaction's success.

Claus and co-workers, in their examination of acrolein hydrogenation on $\mathrm{Au} / \mathrm{TiO}_{2}$ and $\mathrm{Au} / \mathrm{ZrO} 2$ catalysts, concluded that anionic gold and the support acted together to produce the active site for allyl alcohol production $(323,462)$. As in Heiz's model, electrons trapped at paramagnetic F-centers (oxygen vacancies) can be transferred to the gold particles. The electron rich gold particles may then have heightened interactions with various adsorbates. Correlating XAFS data with catalyst activity, Kageyama et al found that the most active catalysts also had the highest degree of Ti-Au coordination (463). It was proposed that Au species were anchored in the surface of $\mathrm{TiO}_{2}$ and that the higher catalytic activity may be the result of charge transfer from Ti to give negatively charge gold particles. Manzolli et al, in their study comparing $\mathrm{Au} / \mathrm{TiO}_{2}$ and $\mathrm{Au} / \mathrm{ZrO}_{2}$, concluded that the higher activity of the titania supported catalyst resulted from the presence of oxygen vacancies which donate electrons to small gold particles (374).

Based on a combination of XPS and reactivity data, Horvath et al proposed a mechanism whereby gold supported on iron oxide which is originally cationic becomes neutral or even anionic after oxidation due to a charge transfer from the support to the gold particle (464). TEM data showed that the particle size distribution was essentially unchanged after oxidation indicating that the binding energy shift observed probably arose from a change in the chemical relationship between the gold and the support. Andreeva and co-workers have also invoked the idea of charge transfer from the support to the gold particles to explain the strong synergistic effects observed in the oxidation of benzene over $\mathrm{Au} / \mathrm{V}_{\mathrm{x}} \mathrm{O}_{\mathrm{y}} / \mathrm{TiO}_{2}$ catalysts (465).

Nieuwenhuys' group observed that while preoxidized samples of $\mathrm{Au} / \mathrm{TiO}_{2}$ were initially inactive, their activity increased following exposure to $\mathrm{CO}$ and $\mathrm{O}_{2}$ (376). On the other hand, prereduced samples exhibited a high activity from the reaction onset. However, extensive reduction of catalysts has been shown to be detrimental to their activity in many cases $(423,466-468)$.

The presence of special sites created by the metal support interface is a common explanation invoked in the theory of catalytic mechanisms (469). Since neither bulk gold nor bulk 
titania are active (or are at least significantly less active than $\mathrm{Au} / \mathrm{TiO}_{2}$ ) for low temperature CO oxidation (403), some have theorized that special sites created by the interface must play a key role in the catalysis $(226,403,430,470-472)$. Strong evidence for such sites has been presented by Vannice and co-workers by their use of inverse catalysts of titania particles deposited on gold substrates. When depositing $\mathrm{TiO}_{x}$ on gold powder, appreciable activity was observed (403), leading the authors to conclude that activity has no relationship to quantum size effects, but rather is primarily a function of the metal-support interface. Efforts to identify specific adsorption sites for $\mathrm{CO}$ on $\mathrm{Au} / \mathrm{TiO}_{2}$ that arise from the interface proved inconclusive using TPD (471). This phenomena is not unique to $\mathrm{Au} / \mathrm{TiO}_{2}$, as $\mathrm{Fu}$ et al have seen similar synergism in $\mathrm{Au} / \mathrm{CeO}_{2}$ catalysts (365). From their results, the size of the gold particles did not appear to play a role in the catalyst's activity. However, the size of the $\mathrm{CeO}_{2}$ crystals was a critical parameter. Guczi and co-workers have proposed a mechanism whereby there must be contact between gold and the active oxide phase based on their study of various $\mathrm{Au} / \mathrm{FeO}_{x}$ catalysts (472). They speculated that the interface between amorphous iron oxide and gold is electron rich, resulting in an ideal place for CO adsorption and oxidation. However, in our own work, we also failed to see unique adsorption sites for CO that must be ascribed to the metal-support interface (302,370). However, FTIR experiments of $\mathrm{CO}$ adsorption have shown that on many surfaces adsorption features are seen at 2090-2105 cm-1, which are generally outside the normal range for metallic gold $\left(2105-2125 \mathrm{~cm}^{-1}\right)$ (473). Therefore these features have been assigned to particle edges at the support interface. It remains unclear, however, how these adsorption sites are related to the unique catalysis by gold.

Recent calculations by Hammer and Molina for CO oxidation by a 34 atom gold cluster on $\mathrm{MgO}$ revealed that the support serves to assist in the stabilization of the reaction intermediate (474). Various types of interfaces were tested for adsorption of oxygen and $\mathrm{CO}$ and for their reaction as depicted in Fig 28 a-c. CO was found to bond to the gold cluster at a low coordinated site near the edge. CO adsorption at the interface of between the support and clusters with a high degree of wetting (as in 28a) was found to be disadvantageous due to steric effects as the CO was repulsed by the support. Like Sanchez et al (247), a mechanism involving molecular oxygen was favored since the barrier to dissociation of oxygen is too great (more than $1 \mathrm{eV}$ ). Oxygen was found to bond to both the cluster and support, ultimately forming a CO-O-O complex at the cluster's edge with the most favorable arrangement shown in Figure 28b. When the complex rearranges as depicted in Fig. 28d, then the barrier to reaction is lowered significantly and $\mathrm{CO}_{2}$ is readily formed. The second oxygen remaining on the gold particle is even

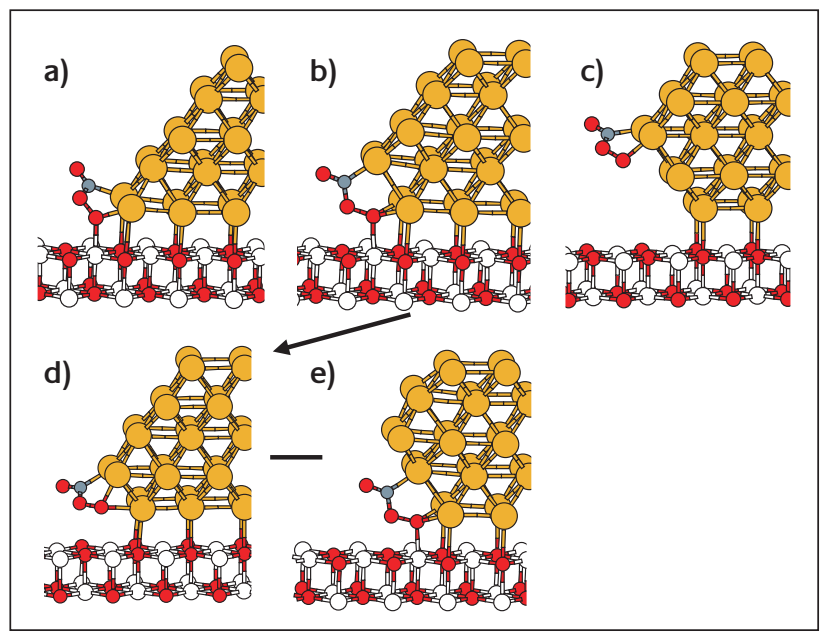

Figure 28

Proposed $\mathrm{CO}-\mathrm{O}_{2}$ binding configurations on various shaped $\mathrm{Au}_{34}$ Clusters on $\mathrm{MgO}(100)$. See text for details. (474)

more easily reacted with CO. Fig. 28 e shows an alternate arrangement of the reaction at the interface. However, in this case the adsorbates are not bound to lowly coordinated gold as in $28 \mathrm{~b}$ or $\mathrm{d}$ so the reaction is less favorable.

Ajo et al have shown that propylene may have unique adsorption sites on the particle-support interface (475). For gold particles with an estimated average thickness of $1.5 \mathrm{ML}$ on $\mathrm{TiO}_{2}(110)$, two distinct peaks ( $150 \mathrm{~K}, 240 \mathrm{~K}$ ) were seen from propene desorption at low coverages. The low temperature peak was assigned to propene desorption from bulk gold surfaces (as seen by Davis and Goodman (104)). As coverage was increased, a third peak emerged at $190 \mathrm{~K}$ which corresponded to propene desorption from titania. Ajo et al speculated that the high temperature feature resulted from sites on the particle-support interface. At low coverages, propene was adsorbed directly onto the particles and was effectively trapped there by propene that adsorbed on the titania and then migrated to the interface. Thicker gold islands on $\mathrm{TiO}_{2}(110)$ exhibited similar behavior, although the loss of the high temperature tail to the TPD spectra was observed. The authors ascribed this high temperature tail to propene desorption from the interface of the support with one monolayer thick particles, which were no longer present for higher gold loadings. It was therefore estimated that the desorption energy from such sites was about 20\% higher than that from edge sites of bulk gold particles.

Schüth and co-workers have employed a novel solution technique to develop highly active gold particles that have limited contact with the support (476). The catalyst was prepared by depositing $\mathrm{AuCl}_{4}$ on an alkylammonium functionalized silica support. As the support is essentially covered with an organic, the metal-support interaction should be negligible and therefore, the authors reject hypotheses that involve special sites created by the metal support 
interface. Noting that active catalysts have been created on a variety of supports, they assert that gold particles must only be sufficiently small in order to display good activity.

As mentioned previously, the adsorption site of oxygen is largely a mystery. Oxygen is known to adsorb readily on oxygen vacancies on metal oxide supports such as titania $(477,478)$. However, oxygen does not merely fill vacancies and as both molecular and atomic oxygen can coexist on the titania surface under the right circumstances (479), the determination of the reactive form of oxygen is complex (as discussed below). As indicated in Fig 26, Haruta and co-workers have described mechanisms whereby oxygen is adsorbed on the support (either dissociatively or molecularly) and then is transported to the particle-support interface where it reacts with CO. Using FTIR, Magkoev et al observed that for gold deposited on a highly reduced $\mathrm{TiO}_{x}$ support the rate of disappearance of $\mathrm{CO}$ was far greater than for $\mathrm{Au} / \mathrm{TiO}_{2}$ upon exposure to oxygen (480). The authors rationalized this by proposing a mechanism whereby the presence of oxygen vacancies greatly facilitated the adsorption of oxygen and subsequent reaction. Complementary XPS experiments revealed that the $\mathrm{Au} / \mathrm{TiO}_{2}$ catalytic surface could be reduced following prolonged exposures to $\mathrm{CO}$ at $500 \mathrm{~K}$. Since reduction did not occur in the absence of gold, the authors concluded the presence of the $\mathrm{Au} / \mathrm{TiO}_{2}$ interface must act as the active site for catalysis. However, from their study comparing the production of $\mathrm{CO}_{2}$ from $\mathrm{Au} / \mathrm{TiO}_{2}$, Au powder and $\mathrm{TiO}_{2}$ powder, lizuka et al observed that oxidized $\mathrm{Au}$ powder produced about 200 times the amount of $\mathrm{CO}_{2}$ as oxidized $\mathrm{TiO}_{2}$ powder after exposure to $\mathrm{CO}$ (481). Therefore since it appeared that oxygen reacts only with great difficulty on $\mathrm{TiO}_{2}$, the authors concluded that oxygen activation (whether dissociative or not) must take place on the surface of gold particles or at the particle-support interface.

Another factor to consider of course with regard to the activity of gold concerns the presence of impurities. It has been shown, in numerous examples (482-484), that bimetallic particles involving gold are superior to gold alone. Therefore, it stands to reason that impurities present in gold actually have a promoting effect in catalysis. In fact, as previously discussed, such impurities have been found to facilitate oxygen adsorption and dissociation (96-100). lizuka and co-workers recently presented evidence that the reactivity of $\mathrm{Au}$ powder could be tied directly to the concentration of silver at the surface (485). Although, gold possesses a very low surface energy, impurities may be stabilized at the surface in a reactive environment and thereby contribute significantly to catalysis (486).

It should be clear that from the above discussion that different reactions may involve different sites and that the principles controlling the chemistry for any particular reaction may not be applicable to others involving very similar catalysts. In other words, the "magic" of gold nanoparticles cannot be simply tied to one particular attribute or active site. General statements regarding the origin of gold's astonishing activity should therefore be avoided as the reactivity for any given reaction is likely a result of a combination of factors. In fact, as will be discussed in the next section, multiple reaction pathways involving multiple active sites may exist even for a simple reaction such as $\mathrm{CO}$ oxidation.

\subsection{Mechanisms of reaction}

Another compelling problem in gold chemistry is an understanding of the catalytic mechanisms. For $\mathrm{CO}$ oxidation, there are at least three mechanisms which have received support.

1 CO reacts with atomic oxygen

2 co reacts with molecular oxygen

3 The reaction proceeds through a complex scheme involving surface hydroxyls.

Of course, as with any scientific problem of importance, the situation is not so simple. As one can see from our discussion of the active site there exist numerous variations and possibilities. Some researchers have even proposed that the mechanism could be different for different types of supports $(226,413,487)$ or that multiple pathways are concurrently active.

Atomic oxygen on the surface of gold single crystals has clearly proven to be capable of oxidizing CO and catalyzing other reactions as discussed in Section 2.3. However, the critical question remains: how does oxygen dissociate on gold surfaces. As mentioned in Section 4, the dissociation of oxygen even on highly stepped surfaces is an extremely endothermic process (197). Therefore, it must be believed that the barrier to dissociation of oxygen on very small supported particles is substantially lowered, perhaps due to electronic effects. As of yet there is no direct evidence that small gold particles dissociate oxygen although, recent calculations of $\mathrm{O}_{2}$ on a ten atom cluster indicate this may be possible when gold is relatively uncoordinated (221). In addition, the adsorption of atomic oxygen appears to be rather strongly dependent on particle size indicating that the dissociation of oxygen is also likely to be favorably influenced by particle size (196).

Although $\mathrm{CO}$ oxidation occurs through dissociated oxygen atoms on other metal surfaces such as Pt (488), mechanisms involving molecular oxygen have been proposed for gold catalysts by many researchers $(226,247,375,377)$. Since CO oxidation has previously been shown to occur with molecular oxygen on silver surfaces (489), it seems logical that gold may also favor such a reaction pathway. However, as molecular oxygen does not adsorb readily either dissociatively or intact on gold surfaces, the mechanism has proven to be difficult to elucidate.

A remarkable feature of the $\mathrm{CO}$ oxidation reaction catalyzed by small gold particles is the incredibly low temperatures by which these reactions are possible. For 
example, highly dispersed $\mathrm{Au} / \mathrm{TiO}_{2}$ catalysts have shown activity down to $209 \mathrm{~K}$ (490, for example) and gold clusters have shown reactivity at liquid nitrogen temperatures $(169,185)$. Although it is known that bulk gold is a catalyst for CO oxidation at high temperatures $\left(300^{\circ} \mathrm{C}\right)(491-493)$, this reaction may occur via a completely different mechanism than the low temperature reaction (or even catalyzed by impurities). It has been argued that the fact that CO oxidation on small gold particles occurs at low temperatures with very low activation energies implies that it cannot proceed through a mechanism involving a highly energetic process such as oxygen dissociation $(220,474)$. Schubert et al (226) and Iwasawa and co-workers $(375,377,494)$ have reported that ${ }^{36} \mathrm{O}_{2}$ and ${ }^{32} \mathrm{O}_{2}$ demonstrated no mixing on the catalyst surface. Furthermore, when reacting $\mathrm{C}^{16} \mathrm{O}$ with ${ }^{36} \mathrm{O}_{2}$ the only reaction product seen was $\mathrm{C}^{16} \mathrm{O}^{18} \mathrm{O}$ with no double labeled $\mathrm{CO}_{2}$ of either type. These results indicate that lattice oxygen was not participating in the reaction in any fashion and suggest that oxygen did not dissociate readily on the surface. This would seem to argue against any type of Marsvan Krevelen mechanism (454) as has been proposed in some work $(487,496,497)$. Schubert et al proposed a mechanism in which oxygen adsorbed at the metal/support interface reacts with CO through one of two routes (226). In the first route, molecular oxygen adsorbs on the support surface before migrating to the interface where it can dissociate and react with $\mathrm{CO}$ through a spillover process. In the second mechanism, a direct reaction between $\mathrm{CO}$ and molecular oxygen is suggested to occur through a noncarbonate-like transition state. While Behm's group favors the first of these mechanisms, Iwasawa's group favors the latter $(375,377)$. Hao et al observed $\mathrm{O}_{2}^{-}$ions on the surface of $\mathrm{Au} / \mathrm{ZnO}$ using EPR after exposure to oxygen at room temperature (498). Upon introduction of $\mathrm{CO}$, the evolution of $\mathrm{CO}_{2}$ was promptly observed until the $\mathrm{O}_{2}^{-}$species was completely consumed. This led the authors to conclude that the $\mathrm{O}_{2}{ }^{-}$species itself is the active species or $\mathrm{O}_{2}{ }^{-}$is a precursor to the active species. However, in Haruta's examination of $\mathrm{Au} / \mathrm{TiO}_{2}$ catalysts with EPR, it was possible to generate $\mathrm{O}_{2}{ }^{-}$on titania in the absence of gold, which seems to speak against a mechanism requiring the presence of $\mathrm{O}_{2}{ }^{-}$(499). Furthermore, the authors note that after the catalyst has been exposed to both $\mathrm{O}_{2}$ and $\mathrm{CO}$, it can no longer stabilize $\mathrm{O}_{2}{ }^{-}$species on the surface, although it can be argued that the anionic oxygen is quickly reacted with $\mathrm{CO}$ such that its observation is precluded.

Stable carbonate intermediates have been observed on the surface in several IR studies of real catalysts $(191,358,375,377,403,421,422,430,473)$. Originally thought to be a key part of the mechanism $(191,473)$, the prevailing view today is that $\mathrm{CO}_{3}$ is a spectator species in the reaction $(3,4,496)$ and that it can even act to deactivate the catalyst by blocking reaction sites $(382,467,500-503)$. However, the transition could very well be a non-symmetrical carbonate such that preferential cleavage of the O-OCO bond is conceivable as has been proposed by both theorists and experimentalists $(226,247,474)$.

Interestingly, although it has been hypothesized that the adsorption of oxygen, either dissociatively or molecularly, is the rate limiting step due to the extreme difficulty with which gold adsorbs oxygen, data from real catalysts seem to point in other directions. Haruta and others have consistently observed higher reaction orders for oxygen as for carbon monoxide. However, these reaction orders are frequently quite close to zero which would imply that both species are adsorbed to saturation on the surface at all times during the reaction and that the surface reaction itself is actually the rate limiting step $(4,191)$. Bethke and Kung found that gold catalysts with larger particles were not only less active than those with smaller particles but also less selective with respect to $\mathrm{CO}$ oxidation when using a $\mathrm{CO} / \mathrm{H}_{2}$ mixture (504). Since $\mathrm{H}_{2}$ oxidation became favored for larger particles, one could conclude that the rate limiting step is not the dissociation of oxygen but rather the competition between hydrogen and $\mathrm{CO}$ adsorption that controlled the reaction rate.

Many studies of real catalysts have observed that the presence of water may enhance catalyst performance. Boccuzzi and Chorino suggested that water activates the dissociation of oxygen on gold particles and proposed that oxygen reacts with water to produce an oxygen radical available for reaction and two surface hydroxyl groups (505). Bond and Thompson (6), citing the difficulty of oxygen dissociation on gold surfaces, have even proposed reaction mechanisms involving water such as the one given below (and shown in Figure 29).

$$
\begin{aligned}
& \mathrm{CO}_{\text {ad }}+\mathrm{OH}_{\text {ad }} \rightarrow \mathrm{COOH}_{\text {ad }} \\
& \mathrm{O}_{2} \text { ad }+\mathrm{COOH} \text { ad } \rightarrow \mathrm{O}_{2} \mathrm{H}_{\text {ad }}+\mathrm{CO}_{2 \mathrm{~g}} \\
& \mathrm{O}_{2} \mathrm{H}_{\text {ad }}+\mathrm{CO}_{\text {ad }} \rightarrow \mathrm{OH}_{\text {ad }}+\mathrm{CO}_{2 \mathrm{~g}}
\end{aligned}
$$

In this mechanism the requirement of breaking an $\mathrm{O}_{2}$ bond in adsorbed oxygen is no longer necessary, as the $\mathrm{O}-\mathrm{OH}$ bond is cleaved instead. The reaction is conceived via a reaction between a hydroxyl group that has moved from the support to $\mathrm{a} \mathrm{Au}^{3+}$ ion and $\mathrm{CO}$ adsorbed on the gold particle. The resulting carboxylate group reacts with $\mathrm{O}_{2}{ }^{-}$and forms $\mathrm{CO}_{2}$ and $\mathrm{HO}_{2}$. The hydroperoxide can then react with a second carboxylate group which forms $\mathrm{CO}_{2}$ and two surface hydroxyls, completing the cycle.

While some results have indicated that water acts to deactivate gold catalysts $(359,403,432,506)$, the prevailing view is that water, at least in small amounts, substantially improves activity $(3,4,487,507)$. In fact, in an ultra-dry environment, $\mathrm{CO}$ oxidation over $\mathrm{Au} / \mathrm{Mg}(\mathrm{OH})_{2}$ has been shown by Cunningham et al. to exhibit a negative activation energy (508). This led the authors to conclude that a direct reaction 


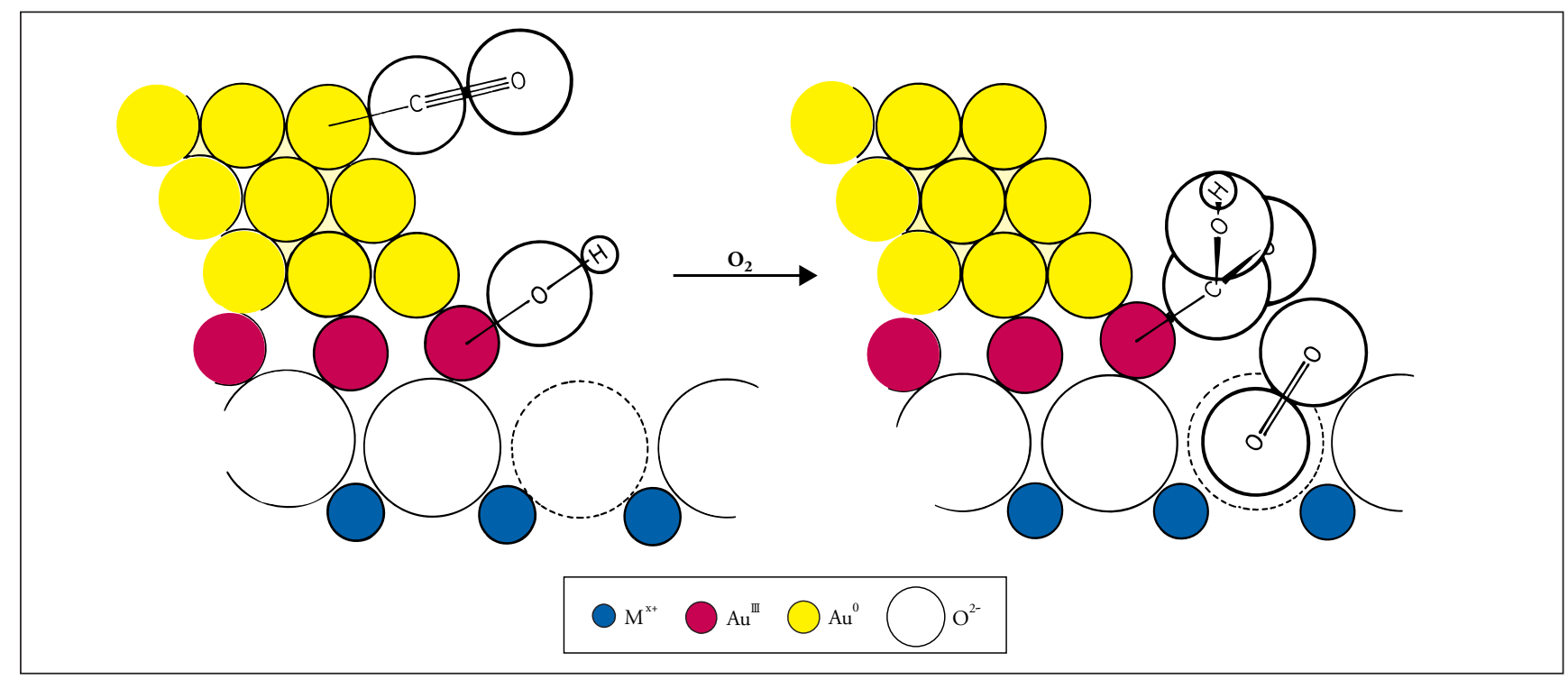

Figure 29

Schematic representation of the early stage of the CO oxidation at the periphery of a gold particle involving the presence of hydroxyl groups and both $A u^{3+}$ and $A u^{0}$. See text for details. (6)

between hydroxyl groups on the surface and CO was a plausible reaction pathway. The presence of gold on the surface has been found to enhance the formation of hydroxyl groups on $\mathrm{Au} / \mathrm{Fe}_{2} \mathrm{O}_{3}$ as compared to the bare support (366).

Haruta and Date have reported that in $\mathrm{CO}$ oxidation over $\mathrm{Au} / \mathrm{TiO}_{2}$, a maximum in activity occurred at $200 \mathrm{ppm} \mathrm{H}_{2} \mathrm{O}$ $(509,510)$ as shown in Figure 30 . At high $(6000$ ppm) or low (0.1 ppm) concentrations of water the reaction rate was found to be a factor of 10 lower at $270 \mathrm{~K}$. The improvement in CO conversion is not merely due to a concurrent water gas shift reaction because as pointed out by Sakurai et al (511) the water gas shift reaction for $\mathrm{Au} / \mathrm{TiO}_{2}$ is not active at room temperature.

Kung and co-workers have also found the presence of water to be beneficial to catalyst durability $(512,513)$. Furthermore they have shown that $\mathrm{Au} / \mathrm{Al}_{2} \mathrm{O}_{3}$ catalysts can be regenerated by either water or hydrogen with exposure to the former being more effective (514). They propose a mechanism similar to Bond and Thompson (6) whereby a CO is inserted in to a Au-OH bond, forming a hydroxycarbonyl on the surface. The hydroxycarbonyl can then react with oxygen as in the mechanism described above or alternatively, decarboxylation to $\mathrm{CO}_{2}$ and $\mathrm{Au}-\mathrm{H}$ can occur, followed by oxidation of the latter to regenerate the surface hydroxyl. Deactivation is believed to result from formation of carbonate groups which can be removed by reaction with water or hydrogen. Like Bond and Thompson, Kung and co-workers proposed that the presence of cationic gold is necessary for the reaction as cationic gold acts as the adsorption site for hydroxyls. Kung's group has also emphasized that chlorine contamination which displaces hydroxyl groups is a key factor in deactivation of gold catalysts (515).
Another possibility is that the coadsorption of water simply lowers the reaction barrier to CO oxidation. However, as Gong and $\mathrm{Hu}$ have recently pointed out in their examination of the catalytic role of $\mathrm{H}_{2} \mathrm{O}$ on $\mathrm{Pt}(111)$, it is not likely that to be a primary pathway for $\mathrm{CO}$ oxidation as the reaction barrier (at least for $\mathrm{Pt}(111)$ ) is on the same order as the barrier to water desorption (516).

With regard to the debate over the presence of metallic gold or cationic gold as the active species, Bond and Thompson proposed that both $\mathrm{Au}^{3+}$ and $\mathrm{Au}^{0}$ are required (6) as depicted in the schematic of Figure 29. In agreement with this type of mechanism, Gates and co-workers found that in active $\mathrm{CO}$ oxidation gold catalysts on MgO supports, both cationic and neutral gold species were present (517). Other researchers have since lent their support to mechanisms involving the formation of surface formate and the presence of both metallic and oxidic gold $(450,456)$. Bond and Thompson have further speculated that the mechanism may not be the same on all supports (6). Clearly, this is an area that demands more attention and should be an area where contributions from surface science can play a role.

It should be pointed out that CO oxidation is one of simplest reactions catalyzed by gold and that the understanding of other reactions such as the selective oxidation of propene to propylene oxide is even further behind. The reaction is optimized at about $100^{\circ} \mathrm{C}$ and requires the presence of hydrogen without which, the primary products are $\mathrm{CO}$ and $\mathrm{CO}_{2}$ $(10,518,519)$. Higher temperatures lead to the formation of secondary products such as propionaldehyde (520) and $\mathrm{CO}_{2}$ while lower temperatures give very high selectivity although at the expense of activity (518). Particle size appears to be a key 


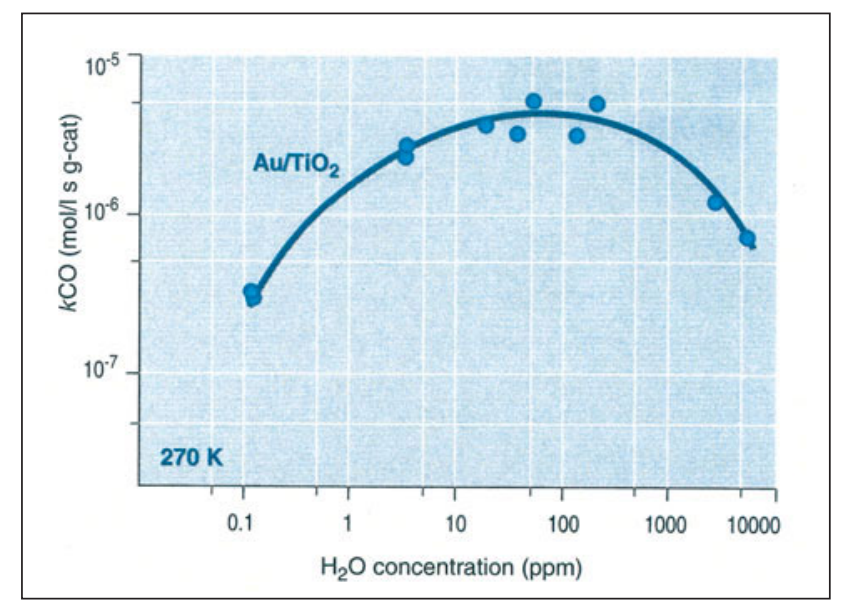

\section{Figure 30}

Effect of the moisture concentration in the reaction gas on the $\mathrm{CO}$ oxidation reaction rate at $270 \mathrm{~K}$ over 1 wt.\% Au/TiO 2 catalyst. (3 (data from 509))

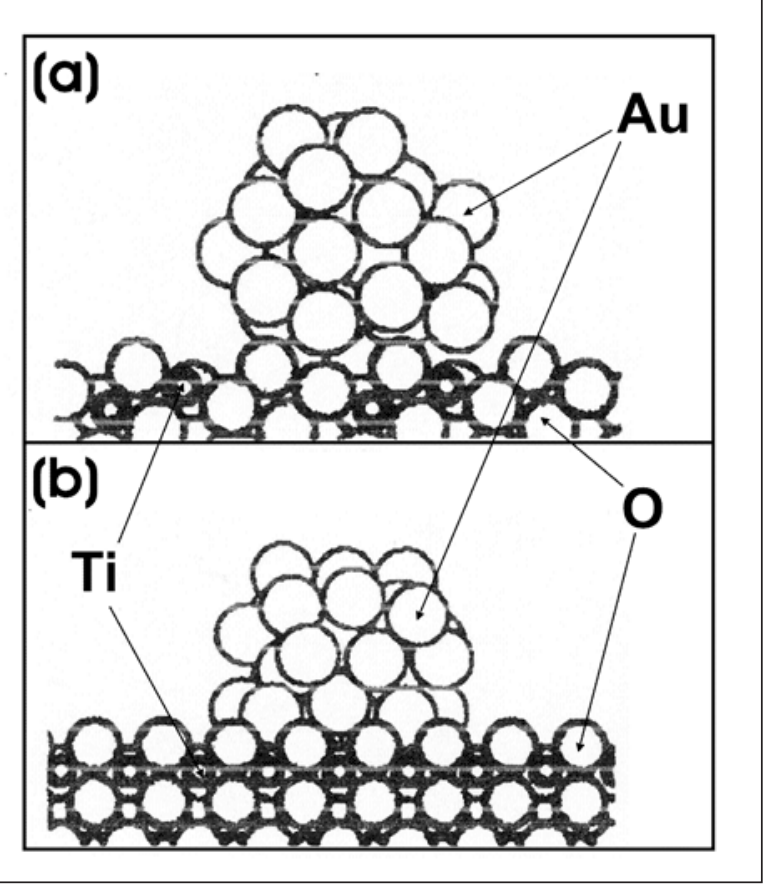

Figure 31

The structure of the Au/titania interface for (a) anatase and (b) rutile demonstrating differences in the spacing between Au and Ti cations. (522)

variable as particles smaller than $2 \mathrm{~nm}$ favor the hydrogenation reaction to propane and large particles (521). The choice of support is of critical importance for selective oxidation of propene as only anatase $\mathrm{TiO}_{2}$ supports and neither rutile nor amorphous $\mathrm{TiO}_{2}$ supports have shown to be active (522). $\mathrm{TiO}_{2}-$ $\mathrm{SiO}_{2}$ mesoporous crystalline structures have proven to be active for this reaction $(425,519,520,523,524)$ as well when possessing the proper Ti:Si ratio (generally below 3:100) $(524,525)$. It is speculated that this may be related to a steric effect whereby Ti cations must be spaced at critical distances $(522,523)$ as catalysts with such mesoporous material supports tend to be less active with increasing Au and Ti content (525). In Fig. 31, the relationship between Au and Ti cations for the rutile and anatase forms of titania are depicted, revealing the differences in spacing between $\mathrm{Ti}$ and $\mathrm{Au}$ (522). As discussed in Molina and Hammer's work with CO oxidation (474), the transition state is probably rather complex therefore very precise interatomic distances are required (523). As in the case of $\mathrm{CO}$ oxidation, some debate exists as to the nature of the active gold species (526). The mechanism is conceived to occur through the presence of a hydrogen peroxide intermediate which acts as the oxidizing agent (527). Theoretical calculations and experimental results alike have shown that formation of $\mathrm{H}_{2} \mathrm{O}_{2}$ is relatively facile over gold surfaces (528-530), lending credence to a mechanism involving hydrogen peroxide. Recent work has also confirmed the presence of a hydroperoxy species on the surface during a $\mathrm{H}_{2}+\mathrm{O}_{2}$ reaction over $\mathrm{Au} / \mathrm{TiO}_{2}$ and it is thought that a similar species may form during propylene epoxidation (531). The use of $D_{2}$ induced an isotope effect, suggesting that the adsorption (or reaction) of hydrogen is the rate limiting step (518). In a recent review, Haruta speculated that after adsorption of oxygen on gold, the particles become electron deficient due to the electronegativity of oxygen and thereby adopt an electronic structure similar to platinum which allows for the facile dissociation of $\mathrm{H}_{2}$ (532). However, the direct hydrogenation of propene to propane, which is observed for particles under $2 \mathrm{~nm}$, is still difficult to explain in the context of such a mechanism.

In addition, very few studies have been performed that address the long-term performance of these catalysts (501,503,533-535). Some results have indicated that agglomeration of gold particles could pose a serious threat to long-term stable activity $(192,394,412,421,476,535)$. Others have attributed the presence $(403,432,506)$ or absence $(507,508,512,513)$ of water as a contributing factor to deactivation. Minico et al ascribed deactivation of $\mathrm{Au} / \mathrm{Fe}_{2} \mathrm{O}_{3}$ to an irreversible reduction of the gold from the 1+ state to metallic gold (378). For the selective oxidation of propylene, $\mathrm{Au} / \mathrm{TiO}_{2}$ catalysts also may experience problems with coking (527) as a result of propoxy decomposition at the acid sites of the titanosilicate support (523). Recently, it has been proposed that alkali metals such as lithium can be used as promoters to guard against sintering (536). While many researchers have reported phenomenal activity or selectivity these catalysts will not enjoy commercial success until they prove to be more robust.

\subsection{Concluding remarks}

Taken from a surface science perspective, real catalyst systems often seem to be poorly characterized and "standard" preparation techniques including the calcinations conditions vary widely from one lab to another leading to 
significant confusion in the analysis of results. Furthermore, experiments sometimes appear to be taken in an unsystematic manner and general trends are therefore often obscured. As one can readily appreciate from the preceding discussion in Section 6, in many cases the various results seem to be directly contradictory and therefore the reaction mechanism and active site for catalysis by gold nanoparticles remain unknown. It is also well known (but not often discussed) that the reproducibility of results of gold catalysts is of particular difficulty (426). Again, this largely stems from the lack of standardized catalyst preparation methods and the lack of proper characterization. Recently, certain standard catalyst preparations have been offered by Süd Chemie Japan in an effort to make comparison of results between various laboratories more meaningful (537). In the opinion of the current authors, surface science studies of well characterized model systems can make strong contributions in this regard as well. The approach we endorse involves the use of model systems whereby the addition of complexity in a stepwise manner allows one to easily identify the effects behind any changes in the chemistry observed. One criticism of surface science experiments is that the conditions under which these experiments are performed, i.e. ultrahigh vacuum, in no way replicate those conditions that the real catalyst must endure. In recent years, so called "high pressure" (1 mbar-1 bar) techniques such as SFG and in situ STM have been utilized in an effort $(235,538)$ to close the well-known pressure gap. Clearly, more experiments are required on model gold catalyst systems in reactive environments in order to better understand the complex chemistry of gold nanoparticles.

As we have previously discussed, a debate exists as to the nature of the active site and the mechanism. In general, $\mathrm{CO}$ is thought to adsorb on the gold particles themselves, probably on edge or step sites $(3,4)$. The adsorption site of oxygen is less clear, although mechanisms involving oxygen adsorption on the support or at the particle-support interface are frequently invoked. Reaction is often conceived to occur at the interface and the nature of the reactive oxygen is still a mystery. In our opinion, molecular oxygen is likely to be the reactive species as theoretical calculations $(197,216,247,474)$ consistently predict very high barriers to dissociation of oxygen, even for highly uncoordinated systems. Mechanisms involving hydroxyl groups largely stem from empirical evidence describing the improvement of catalytic performance in the presence of traces of water $(507,509)$. Similar claims for a mechanism involving hydroxyls have been made previously for $\mathrm{CO}$ oxidation on alumina supported Pd (539). However, we have demonstrated in our lab that $\mathrm{CO}_{2}$ does not form by $\mathrm{CO}$ exposure to Pd particles deposited on either pristine or hydroxylated alumina films (540).

Therefore, we would support a mechanism in which molecular oxygen is adsorbed on highly uncoordinated sites on gold and reacts with $\mathrm{CO}$, which is also adsorbed on gold. Recent work in our lab on the $\mathrm{Au} / \mathrm{FeO}$ system indicates that quantum size effects alone do not control the adsorption of $\mathrm{CO}$ on gold nanoparticles and therefore suggest that the thickness of gold particles is not the critical parameter in CO oxidation (370). The requirement of a particular size therefore seems associated with the presence of highly uncoordinated atoms as has been demonstrated by Bahn et al (222). The increase of reactivity with decreasing coordination is not in itself surprising. However, the large differences in the chemistry of gold as its coordination number is reduced is exceptional and appears related to relativistic effects which do not arise in many other cases (34). The presence or absence of oxidic or anionic gold does not appear to be an important factor in CO oxidation, although there is evidence that $\mathrm{Au}^{+}$could be critical for other reactions. As both theory and experiment have shown a strong preference for nucleation of gold on defects on oxide surfaces, the defect structure of the support is, therefore, critical in stabilization of the geometry required for the reaction. While it seems clear with the use of supports such as $\mathrm{CeO}_{2}$ and $\mathrm{Fe}_{2} \mathrm{O}_{3}$ that a Mars-van Krevelen mechanism does come into play under certain reaction conditions, this is not the predominant mechanism. In conclusion, we believe that surface science studies of well-defined model systems can make strong contributions to the understanding of the unique reactivity of nanosized gold. It is our hope and intention to encourage other surface scientists to take on the challenges posed by this fascinating catalysis.

\section{Acknowledgements}

The authors would like to gratefully acknowledge Erika Popovich and Matthias Nachitzki for technical support. Furthermore, we would like to thank Prof. Claude Henry for his careful and thoughtful reading of the manuscript. We also are indebted to the Training Mobility and Research Project "Reactivity of clean and modified oxide surfaces" headed by Prof. Henry (TMR Marseille) and Training Mobility and Research Project "Catalysis by Gold” headed by Prof. Graham Hutchings (TMR AURICAT) for financial support as well as scientific discussions.

\section{About the Authors}

Drs. Randall Meyer, Celine Lemire, Shamil K. Shaikhutdinov work in Professor Hans-Joachim Freund's group where they study the interaction and reaction of molecules with model catalyst systems. Details can be found on the web site http:/www.fhiberlin.mpg.de/cp.new/sr/. Professor Freund studied physics and 
chemistry at the University of Cologne. He subsequently worked at the University of Pennsylvania, the University of ErlangenNürnberg and the Ruhr-Universität at Bochum before moving to the Fritz Haber Institute in 1995.

\section{References}

1 M. Haruta, Catalysis Today, 1997, 36, 153

2 M. Haruta, and M. Date, Appl. Catal. A, 2001, 222, 427

3 M. Haruta, CATTECH, 2002, 6, 102

4 M. Haruta, Chemical Record, 2003, 3, 75

5 G.C. Bond and D.T. Thompson, Catalysis Reviews: Science and Engineering, 1999, 41, 319

6 G.C. Bond and D.T. Thompson, Gold Bull., 2000, 33, 41

7 G.C. Bond, Catal. Today, 2002, 72, 5

8 D.T. Thompson, Appl. Catal. A, 2003, 243, 201

9 M. Haruta, N. Yamada, T. Kobayashi and S. lijima, J.Catal., 1989, 115, 301

10 T. Hayashi, K. Tanaka and M. Haruta, J. Catal., 1998, 178, 566

11 D. Andreeva, V. Idakeiv, T. Tabakova, A. Andreev and R. Giovanoli, Appl. Catal. A: General, 1996, 134, 275

12 A. Ueda and M. Haruta, Gold Bull., 1999, 32, 3

13 G.C. Bond, P.A. Sermon, G. Webb, D. Buchanan and P.B. Wells, J. Chem. Commun., 1973, 444

14 H. Sakuri and M. Haruta, Catal.Today, 1996, 29, 361

15 T. Aida, R. Higuchi and H. Niiyama, Chem. Lett., 1990, 2247

16 G. Hutchings, Catal. Today, 2002, 72, 11

17 G.J. Hutchings, J. Catal., 1985, 96, 292

18 B. Nkosi, M.D. Adams, N.J. Coville and G.J. Hutchings, J. Catal., 1991, 128, 366

19 Gold Bull., 2003, 36, 21

20 D. J. Gulliver, and J. S. Kitchen, EU Patent \#654301, BP. Chem. Int. Ltd., 1995

21 G. Srinivas, J. Wright, C.S. Bai, and R. Cook, Studies in Surface Science and Catalysis, 1996, 101, 427

22 M.M. Schubert, M.J. Kahlich, H.A. Gasteiger and R.J. Behm, J. Power Sources, 1999, 84, 175

23 D. Cameron, R. Holliday and D. Thompson, Journal of Power Sources, 2003, 118, 298

24 T.V. Choudhary and D.W. Goodman, Catalysis Today, 2002, 77, 65

25 A.S.K. Hashmi, Gold Bull. 2003, 36, 3

26 J.H. Teles, S. Brode and M. Chabanas, Angew. Chem. Int. Ed. 1998, 37, 1415

27 G.C. Bond, Journal of Molecular Catalysis A, 2000, 156, 1

28 K. Pitzer, Acc. Chem. Res., 1979, 12, 271

29 P. Pyykkö and J.P. Desclaux, Acc. Chem. Res., 1979, 12, 276

30 P. Pyykkö, Angewandte Chemie Intl. Ed., 2002, 41, 3573

31 H. Tsai, E. Hu, K. Perng, M. Chen, J.C. Wu and Y.S. Chang, Surf. Sci., 2003, 537, L447

32 H. Schmidbaur, Gold Bull., 2000, 33, 3

33 H. Häkkinen, M. Moseler and U. Landman, Phys. Rev. Lett., 2002, 89, 033401
34 T. Nautiyal, S.J. Youn and K.S. Kim, Phys. Rev. B, 2003, 68, 033407

35 R. Masel, Principles of Adsorption and Reaction on Solid Surfaces, John Wiley \& Sons, 1996, 38

36 J.V. Barth, H. Brune, G. Ertl and R.J. Behm, Phys. Rev. B, 1990, 42, 93

37 M.A. van Hove, R.J. Koestner, P.C. Stair, J. P. Bibérian, L.L. Kesmodel, I. Bartos and G. Somorjai, Surf. Sci., 1981, 103, 189

38 U. Harten, A.M. Lahee, J.P. Toennies and C. Wöll, Phys. Rev. Lett., 1985, 54, 2619

39 G.O. Pötschke and R.J. Behm, Phys. Rev. B, 1991, 44, 1442

40 V. Heine and L.D. Marks, Surf. Sci., 1986, 165, 65

41 A.R. Sandy, S.G.J. Mochrie, D.M. Zehner, K.G. Huang and D. Gibbs, Phys. Rev. B, 1991, 43, 4667

42 W. Haiss, D. Lackey, J.K. Sass and K.H. Besocke, J. Chem. Phys., 1991, 95, 2193

43 K.F. Peters, P. Steadman, H. Isern, J. Alvarez and S. Ferrer, Surf. Sci., 2000, 467, 1007

44 J.V. Barth, R.J. Behm and G. Ertl, Surf. Sci., 1994, 302, L319

45 L. Huang, J. Chevrier, P. Zeppenfeld and G. Cosma, Appl. Phys. Lett., 1995, 66, 935

46 L. Huang, P. Zeppenfield, J. Chevrier and G. Comsa, Surf. Sci.,1996, 352, 285

47 J. Chevrier, L. Huang, P. Zeppenfeld and G. Comsa, Surf. Sci.,1996, 355, 1

48 Y. Uchida, X. Bao, K. Weiss, and R. Schlögl, Surf. Sci., 1998, 401, 469

49 L. Ortega, L. Huang, J. Chevrier, P. Zeppenfeld, J. M. Gay, F. Rieutord, and G. Comsa, Surf. Rev. Lett., 1997, 4, 1315

50 D.G. Fedak and N.A. Gjostein, Acta Metallurgica, 1967, 15, 827

51 K. Christmann and G. Ertl, Z. Natur. 1976, 28a, 1144

52 M. Salmeron and G.A. Somorjai, Surf. Sci., 1980, 91, 373

53 T. Gritsch, D. Coulman, R.J. Behm and G. Ertl, Surf. Sci., 1991, 257, 297

54 W. Moritz and D. Wolf, Surf. Sci., 1979, 88, L29

55 I.K. Robinson, Phys. Rev. Lett., 1983, 50, 1145

56 J.K. Gimzewski, R. Berndt and R.R. Schlittler, Phys. Rev. B, 1992, 45, 6844

57 W. Moritz, and D. Wolf, Surf. Sci., 1985, 163, L655

58 K.M. Ho and K.P. Bohnen, Phys. Rev. Lett., 1987, 59, 1833

59 J.C. Campuzano, M.S. Foster, G. Jennings, R.F. Willis and W. Unertl, Phys. Rev. Lett., 1985, 54, 2684

60 R. Koch, M. Sturmat and J.J. Schulz, Surf. Sci., 2000, 454, 543

61 J.M. Gottfried, K.J. Schmidt, S.L.M. Schroeder and K. Christmann, Surf. Sci., 2003, 536, 206

62 J. de la Figuera, M.A. González, R. García-Martínez, J.M. Rojo, O.S. Hernán, A.L. Vázquez de Parga and R. Miranda, Phys. Rev. B, 1998, 58, 1169

63 D.G. Fedak and N.A. Gjostein, Surf. Sci., 1967, 8, 77

64 H. Melle, and E. Menzel, Zeitschrift für Naturforschung, 1978, 33a, 282

65 G.K. Binnig, H. Rohrer, C. Gerber and E. Stoll, Surf. Sci., 1984, 144, 321

66 K. Yamazaki, K. Takayanagi, Y. Tanishiro and K. Yagi, Surf. Sci., 1988, 199, 595

67 N. Wang, Y. Uchida and G. Zehmpfuhl, Surf. Sci., 1993, 284, L419

68 K. Takayanagi, Y. Tanishoro, K. Kobayashi, K. Akiyama and K. Yagi, Japanese Journal of Applied Physics,1987, 26, L957 
K.H. Rieder, T. Engel, R.H. Swendsen and M. Manninen, Surf. Sci., 1983, 127,223

70 J.F. Annett and J.E. Inglesfield, Journal of Physics: Condensed Matter, 1989, 1, 3645

71 N. Takeuchi, C.T. Chan and K.M. Ho, Phys. Rev. Lett., 1989, 63, 1273

72 N. Takeuchi, C.T. Chan and K.M. Ho, Phys. Rev. B, 1991, 43, 14363

73 L.D. Marks and D.J. Smith, Surf. Sci., 1985, 157, L367

74 J.F. Wendelken and D.M. Zehner, Surf. Sci., 1979, 71, 178

75 S.G.J. Mochrie, D.M. Zehner, B.M. Ocko and D. Gibbs, Phys. Rev. Lett., 1990, 64, 2925

76 D. Gibbs, B.M. Ocko, D.M. Zehner and S.G.J. Mochrie, Phys. Rev. B, 1990, 42, 7330

77 J.V. Barth, R. Schuster, R.J. Behm and G. Ertl, Surf. Sci., 1994, 302, 158

78 S.LM. Schroeder, A. Neumann, T. Solomun, P. Lenz-Solomun and K. Christmann, Surf. Sci., 1995, 337, 285

79 B.M.W. Trapnell, Proceedings of the Royal Society A, 1952, 218, 566

80 J.J. Stephan and V. Ponec, J. Catal., 1976, 42, 1

81 A.M. Bradshaw and J. Pritchard, Proceedings of the Royal Society A, 1970, 316, 169

82 P. Dumas, R.G. Tobin and P.L. Richards, Surf. Sci., 1986, 171, 579

83 C. Ruggiero and P. Hollins, Journal of the Chemical Society, Faraday Transcriptions, 1996, 92, 4829

84 C. Ruggiero and P. Hollins, Surf. Sci., 1997, 377, 583

85 T. Gritsch, D. Coulman, R.J. Behm and G. Ertl, Phys. Rev. Lett., 1989, 63, 1086

86 P. Fenter and T. Gutafsson, Phys. Rev. B, 1988, 38, 10197

87 D.A. Outka and R.J. Madix, Surf. Sci., 179 (1987) 351

88 A. Sandell, P. Bennich, A. Nilsson, B. Hernnäs, O. Björneholm and N. Mårtensson, Surf. Sci., 1994, 310, 16

89 K. Dückers, and H.P. Bonzel, Surf. Sci., 1989, 213, 25

90 D.C. Meier, V. Bukhtiyarov and D.W. Goodman, J Phys Chem B, 2003, 107, 12668

91 G. McElhiney and J. Pritchard, Surf. Sci., 1976, 60, 397

92 Y. Jugnet, F.J. Cadete Santos Aires, C. Deranlot, L. Piccolo and J.C. Bertolini, Surf. Sci., 2002, 521, L639

93 C.W. Olsen and R.I. Masel, Surf. Sci., 1988, 201, 444

94 J. Szanyi, W.K. Kuhn and D.W. Goodman, J. Vac. Sci. Technol. A, 1993, 11, 1969

95 N.D.S. Canning, D. Outka and R.J. Madix, Surf. Sci., 1984, 141, 240

96 M.A. Chesters, and G.A. Somorjai, Surf. Sci., 1975, 52, 21

97 W.R. MacDonald and K.E. Hayes, J. Catal., 1970, 18, 115

98 N.A. Shishakov, J. Phys. Chem. 1960, 64, 1580

99 M.E. Schrader, Surf. Sci., 1978, 78, L227

100 J.J. Pireaux, M. Chtaïb, J.P. Delrue, O.A. Thiry, M. Liehr, and R. Caudano, Surf. Sci., 1984, 141, 211

101 N. Saliba, D.H. Parker and B.E. Koel, Surf. Sci., 1998, 410, 270

102 M.A. Lazaga, D.T. Wickham, D.H. Parker, G.N. Kastanas, and B.E. Koel, Am. Chem. Soc. Symp. Ser., 1993, 523, 90

103 D.H. Parker and B.E. Koel, J. Vac. Sci. Tech. A, 1990, 8, 2585

104 K.A. Davis and D.W. Goodman, J. Phys. Chem. B, 2000, 104, 8557

105 J.M. Gottfried, K.J. Schmidt, S.L.M. Schroeder and K. Christmann, Surf. Sci., 2003, 525, 184
106 J.M. Gottfried, K.J. Schmidt, S.L.M. Schroeder and K. Christmann, Surf. Sci., 2002, 511, 65

107 J.M. Gottfried, N. Elghobashi, S.L.M. Schroeder and K. Christmann, Surf. Sci., 2003, 523, 89

108 T. Bär, T. Visart de Bocarmé, B.E. Nieuwenhuys and N. Kruse, Catal. Lett., 2001, 74, 127

109 A.G. Sault, R.J. Madix and C.T. Campbell Surf. Sci., 1986, 169, 347

110 L. Stobinski, Appl. Surf. Sci., 1996, 103, 503

111 G. N. Kastanas, and B. E. Koel, Appl. Surf. Sci., 1993, 64, 235

112 D. A. Outka, and R. J. Madix, J. Am. Chem. Soc., 1987, 109, 1708

113 D. A. Outka, and R. J. Madix, Surf. Sci., 179 (1987) 361

114 D. Syomin, and B.E. Koel, Surf. Sci., 2002, 498, 53

115 D. Syomin, J. Kim, B.E. Koel and G.B. Ellison, J. Phys. Chem. B, 2001, 105, 8387

116 D. Syomin and B.E. Koel, Surf. Sci., 2002, 498, 61

117 D. Syomin, J. Wang and B.E. Koel, Surf. Sci., 2001, 495, L827

118 J. Wang, H. Busse, D. Syomin and B.E. Koel, Surf. Sci., 2001, 494, L741

119 M.E. Bartram and B.E. Koel, Surf. Sci., 1989, 213, 137

120 T. Solomun, K. Christmann and H. Baumgärtel, J. Phys. Chem., 1989, 93, 7199

121 T. Solomun, H. Baumgärtel and K. Christmann, J. Phys. Chem., 1991, 95, 10041

122 M. Beckendorf, U.J. Katter, H. Schlienz and H.J. Freund, Journal of Physics: Condensed Matter, 1993, 5, 5471

123 C.H. Bartholomew, P.K. Agrawal and J.R. Kratzer, Advances in Catalysis, 1982, 31, 135

124 J.E. Bailie and G.J. Hutchings, Chem. Commun., 1999, 2151

125 J.A. Rodriguez, J. Dvorak, T. Jirsak, G. Liu, J. Hrbek, Y. Aray and C. Conzález, J. Am. Chem. Soc., 2003, 125, 276

126 J. Gottschalck and B. Hammer, J. Chem. Phys., 2002, 116, 784

127 S. Vericat, M.E. Vela, G. Andreasen, R.C. Salvarezza, L. Vázquez and J.A. Martín-Gago, Langmuir, 2001, 17, 4919

128 A.J. Leavitt and T.P. Beebe, Surf. Sci., 1994, 314, 23

129 V. Bondzie, St. J. Dixon-Warren and Y. Yu, J. Chem. Phys., 1999, 111, 10670

130 D.M. Jaffey and R.J. Madix, Surf. Sci., 1991, 258, 359

131 B. Frühberger, M. Grunze and D.J. Dwyer, J. Phys. Chem., 1994, 98, 609

132 R.G. Nuzzo, B.R. Zegarski and L.H. Dubois, J. Am. Chem. SoC., 1987, 109, 733

133 G. Liu, J.A. Rodriguez, J. Dvorak, J. Hrbek and T. Jirsak, Surf. Sci., 2002, 505, 295

134 H. Sellers, Surf. Sci., 1993, 294, 99

135 H. Grönbeck, A. Curioni and W. Andreoni, J. Am. Chem. Soc., 2000, 122, 3839

136 R.G. Nuzzo, L.H. Dubois and D.L. Allara, J. Am. Chem. Soc., 1990, 112, 558

137 D.J. Lavrich, S.M. Wetterer, S.L. Bernasek and G. Scoles, J. Phys. Chem. B, 1998, 102, 3456

138 D.M. Jaffey and R.J. Madix, J. Am. Chem. Soc., 1994, 116, 3012

139 D.M. Jaffey and R.J. Madix, Surf. Sci., 1994, 311, 159

140 D.M. Jaffey and R.J. Madix, J. Am. Chem. Soc., 1994, 116, 3020

141 V. Bondzie, St. J. Dixon-Warren, Y. Yu and L. Zhang, Surf. Sci., 1999, 431, 174 
142 C.F. McFadden, P.S. Cremer and A.J. Gellman, Langmuir, 1996, 12, 2483

143 Z. _ljivan_anin, K.V. Gothelf and B. Hammer, J. Am. Chem. Soc., 2002, 124, 14789

144 J.M. Gottfried, K.J. Schmidt, S.L.M. Schroeder and K. Christmann, Surf. Sci., 2003, 525, 197

145 J.M. Gottfried and K. Christmann, Surf. Sci., submitted

146 I.E. Wachs and R.J. Madix, J. Catal., 1978, 53, 208

147 I.E. Wachs and R.J. Madix, Surf. Sci., 1978, 76, 531

148 A.Paul and B. Bent, J. Catal., 1994, 147, 264

149 A. Paul, M. X. Yang and B.E. Bent, Surf. Sci., 1993, 297, 327

150 M.X. Yang, S.K. Jo, A. Paul, L. Avila, B.E. Bent and K. Nishikida, Surf. Sci., 1994, 325, 102

151 D. Syomin and B.E. Koel, Surf. Sci., 2001, 490, 265

152 D. Syomin and B.E. Koel, Surf. Sci., 2001, 492, L693

153 J. Wang and B.E. Koel, Surf. Sci., 1999, 436, 15

154 K. Kishi, M. Date and M. Haruta, Surf. Sci., 2001, 486, L475

155 S. Dahl, E. Törnqvist and I. Chorkendorff, J. Catal., 2000, 192, 381

156 S. Xinyin, D.J. Frankel, J.C. Hermanson, G.J. Lapeyre and R.J. Smith, Phys. Rev. B, 1985, 32, 2120

157 X.Y. Shen, D.J. Frankel, G.J. Lapeyre and R.J. Smith, Phys. Rev. B, 1986, 33,5372

158 A. Sellidj and B.E. Koel, Phys. Rev. B, 1994, 49, 8367

159 M. fl. Pedersen, S. Helveg, A. Ruban, I. Stensgaard, E. Laegsgaard, J.K. Nørskov and F. Besenbacher, Surf. Sci., 1999, 426, 395

160 P.J. Schmitz, H.C. Kang, W.Y. Leung and P.A. Thiel, Surf. Sci., 1991, 248, 287

161 B. Gleich, M. Ruff and R.J. Behm, Surf. Sci., 1997, 386, 48

162 M. Ruff, S. Frey, B. Gleich and R.J. Behm, Applied Physics A, 1998, 66, S513

163 J.W.A. Sachtler, M.A. Van Hove, J.P. Bibérian and G.A. Somorjai, Phys. Rev. Lett., 1980, 45, 1601

164 M. Okada, M. Nakamura, K. Moritani and T. Kasai, Surf. Sci., 2003, 523, 218

165 F. Besenbacher, I. Chorkendorf, B.S. Clausen, B. Hammer, A.M. Molenbroek, J.K. Nørskov and I. Stensgaard, Science, 1998, 279, 1913

166 P. M. Holmblad, J. Hvolbaek Larsen, and I. Chorkendorff, J. Chem. Phys., 1996, 104, 7289

167 P. Kratzer, B. Hammer and J.K. Nørksov, J. Chem. Phys., 1996, 105, 5595

168 C.J. Baddeley, M. Tikhov, C. Hardacre, J.R. Lomas, and R.M. Lambert, J. Phys. Chem., 1996, 100, 2189

169 H. Huber, D. Mclntosh and G. Ozin, Inorganic Chemistry, 1977, 16, 975

170 D.M. Cox, R.O. Brickman, K. Creegan and A. Kaldor, Material Res. Society Proceedings, 1991, 206, 43

171 D.M. Cox, R. Brickman, K. Creegan and A. Kaldor, Z. Phys. D, 1991, 19, 353

172 T.H. Lee and K.M. Ervin, J. Phys. Chem., 1994, 98, 10023

173 L. Lian, P.A. Hackett and D.M. Rayner, J. Chem. Phys., 1993, 99, 2583

174 K.J. Taylor, C.L. Pettiette-Hall, O. Cheshnovsky and R.E. Smalley, J. Chem. Phys., 1992, 96, 3319

175 M.A. Nygren, P.E.M. Siegbahn, C. Jin, T. Guo and R.E. Smalley, J. Chem. Phys., 1991, 95, 6181

176 J. Li, X. Li, H.J. Zhai and L.S. Wang, Science, 2003, 299, 864

177 B.E. Salisbury, W.T. Wallace and R.L. Whetten, Chem. Phys., 2000, 262, 131
178 D. Stolcic, M. Fischer, G. Ganteför, Y.D. Kim, Q. Sun and P. Jena, J. Am. Chem. Soc., 2003, 125, 2848

179 Y.D. Kim, M. Fischer and G. Ganteför, Chem. Phys. Lett., 2003, 377, 170

180 W.T. Wallace and R.L. Whetten, J. Phys. Chem. B, 2000, 104, 10964

181 W.T. Wallace and R.L. Whetten, European Journal of Physics D, 2001, 16,123

182 J. Hagen, L.D. Socaciu, U. Heiz, T.M. Bernhardt and L. Wöste, European Journal of Physics D, 2003, 24, 327

183 J. Hagen, L.D. Socaciu, M. Elijazyfer, U. Heiz, T.M. Bernhardt and L. Wöste, Phys. Chem. Chem. Phys., 2002, 4, 1707

184 W.T. Wallace and R.L. Whetten, J. Am. Chem. SoC., 2002, 124, 7499

185 L.D. Socaciu, J. Hagen, T.M. Bernhardt, L. Wöste, U. Heiz, H. Häkkinen and U. Landman, J. Am. Chem. Soc., 2003, 125, 10437

186 I. Balteanu, O.P. Balaj, B.S. Fox, M.K. Beyer, Z. Bastl and V.E. Bondybey, Phys. Chem. Chem. Phys., 2003, 5, 1213

187 W.T. Wallace, R.B. Wrywas, R.L. Whetten, R. Mitri_ and V. Bona_i_Kouteck_, J. Am. Chem. Soc., 2003, 125, 8408

188 K. Sugawara, F. Sobott and A.B. Vakhtin, J. Chem. Phys., 2003, 118, 7808

189 B. Hammer and J. Norskov, Nature, 1995, 376, 238

190 M. Mavrikakis, P. Stoltze and J. Nørskov, Catal.Lett., 2001, 64, 101

191 M. Haruta, S. Tsubota, T. Kobayashi, H. Kageyama, M.J. Genet and B. Delmon, J. Catal., 1993, 144, 175

192 M. Valden, X. Lai and D.W. Goodman, Science, 1998, 281, 1647

193 G. Mills, M.S. Gordon and H. Metiu, J. Chem. Phys., 2003, 118, 4198

194 Y. Xu and M. Mavrikakis, J Phys Chem. B, 2003, 107, 9298

195 H. Strømsnes, S. Jusuf, A. Bagatur'yants, O. Gropen, and U. Wahlgren, Theo. Chem. Acc., 2001, 106, 329

196 V.A. Bondzie, S.C. Parker, and C.T. Campbell, J.Vac. Sci. Techn. A, 1999, 17, 1717

197 Z.P. Liu, P. Hu, and A. Alavi, J. Am. Chem. Soc., 2002, 124, 14770

198 F. Furche, R. Ahlrichs, P. Weis, C. Jacob, S. Gilb, T. Bierweiler, and M.M. Kappes, J. Chem. Phys., 2002, 117, 6982

199 H. Häkkinen, B. Yoon, U. Landman, X. Li, H.J. Zhai and L.S. Wang, J. Phys. Chem. A, 2003, 107, 6168

200 J. Wang, G. Wang and J. Zhao, Phys. Rev. B, 2002, 66, 035418

201 H. Häkkinen and U. Landman, Phys. Rev. B, 2000, 62, R2287

202 H. Grönbeck and W. Andreoni, Chem. Phys., 2000, 262, 1

203 J. Zhao, J. Yang and J.G. Hou, Phys. Rev. B, 2003, 67, 085404

204 I.L. Garzón, K. Michaelian, M.R. Beltrán, A. Posada-Amarillas, P. Ordejón, E. Artacho, D. Sánchez-Portal and J.M. Soler, Phys. Rev. Lett., 1998, 81, 1600

205 I.L. Garzón, K. Michaelian, M. R. Beltrán, A. Posada-Amarillas, P. Ordejón, E. Artacho, D. Sánchez-Portal, and J. M. Soler, Eur. Phys. J. D., 1999, 9, 211

206 O. D. Häberlen, S.C. Chung, M. Stener, and N. Rösch, J. Chem. Phys., 1997, 106, 5189

207 C.L. Cleveland, U. Landman, M. N. Shafigullin, P.W. Stephens and R.L. Whetten, Z. Phys. D., 1997, 40, 503

208 G.D Agostino, A. Pinto and S. Mobilio, Phys. Rev. B, 1993, 48, 14447

209 J. Uppenbrink and D.J. Wales, J. Chem. Phys., 1992, 96, 8520

210 C.L. Cleveland, U. Landman, T.G. Schaaff, M.N. Shafigullin, P.W. Stephens and R.L. Whetten, Phys. Rev. Lett., 1997, 79, 1873 
211 L.D. Marks, Rep. Prog. Phys. 1994, 57, 603

212 S. Giorgio, C.R. Henry, C. Chapon, G. Nihoul and J.M. Penisson, Ultramicroscopy, 1991, 38, 1

213 M. Okumura, Y. Kitagawa, M. Haruta and K. Yamaguchi, Chem. Phys. Lett., 2001, 346, 163

214 G. Mills, M.S. Gordon and H. Metiu, Chem. Phys. Lett., 2002, 359, 493

215 D.H. Wells, W.N. Delgass and K.T. Thomson, J. Chem. Phys, 2002, 117, 10597

216 B. Yoon, H. Häkkinen and U. Landman, J. Phys. Chem. A, 2003, 107, 4066

217 A. Franceschetti, S.J. Pennycook, and S.T. Pantelides, Chem. Phys. Lett., 2003, 374, 471

218 S.A. Varganov, R.M. Olson, M.S. Gordon and H. Metiu, J. Chem. Phys., 2003, 119, 2531

219 X. Wu, L. Senapati, S.N. Nayak, A. Selloni, and M. Hajaligol, J. Chem. Phys., 2002, 117, 4010

220 H. Häkkinen and U. Landman, J. Am. Chem. Soc., 2001, 123, 9704

221 N. Lopez and J.K. Norskov, J. Amer. Chem. Soc., 2002, 124, 11262

222 S.R. Bahn, N. Lopez, J.K. Norskov and K.W. Jacobsen, Phys. Rev. B, 2002, 66, 081405

223 H. Ohnishi, Y. Kondo and K. Takayanagi, Nature, 1998, 395, 780

224 N.V. Skorodumova and S.I. Simak, Phys.Rev. B, 2003, 67, 121404

225 S. Chrétien, M.S. Gordon, and H. Metiu, submitted

226 M.M. Schubert, S. Hackenberg, A.C. van Veen, M. Muhler, V. Plzak and R.J. Behm, J.Catal., 2001, 197, 113

227 D.W. Goodman, J. Phys.Chem., 1996, 100, 13090

228 C.T. Campbell, Surf. Sci. Rep., 1997, 27, 1

229 C.R. Henry, Surf. Sci. Rep., 1998, 31, 231

230 C.R. Henry, Catalysis and Electrocatalysis at Nanoparticle Surfaces, Marcel Dekker Inc. 2003, 239

231 P. Gunter, J.W. Niemantsverdriet, F. Ribiero and G. Somorjai, Catalysis Reviews: Science and Engineering, 1997, 38, 77

232 A.K. Santra, and D.W. Goodman, J. Phys. Condens. Matter, 2002, 14, R31

233 H.J. Freund, M. Bäumer and H. Kuhlenbeck, Adv. Catal., 2000, 45, 333

234 M. Bäumer and H.J. Freund, Progr.Surf. Sci., 1999, 61, 127

235 H.J. Freund, Surf. Sci., 2002, 500, 271

236 F. Cosandey and T. Madey, Surf. Rev. Lett., 2001, 8, 73.

237 U. Diebold, Surf. Sci. Rep., 2003, 48, 53

238 T. Bredow and G. Pacchioni, Chem. Phys. Lett., 2002, 355, 417

239 G. Pacchioni, Surf. Rev. Lett., 2000, 7, 277

240 L. Zhang, R. Persaud and T.E. Madey, Phys. Rev. B, 1997, 56, 10549

241 X. Lai, T.P.St. Clair, M. Valden and D.W. Goodman, Progr. Surf. Sci., 1998, 59,25

242 Q. Guo, K. Luo, K.A. Davis and D.W. Goodman, Surface and Interface Analysis, 2001, 32, 161

243 A.K. Santra, A. Kolmakov, F. Yang and D.W. Goodman, Japanese Journal of Applied Physics, 2003, 42, 4795

244 N. Spiridis, J. Haber and J. Korecki, Vacuum, 2001, 63, 99

245 S.C. Parker, A.W. Grant, V. A. Bondzie and C.T. Campbell, Surf. Sci., 1999, 441, 10

246 E. Wahlström, N. Lopez, R. Schaub, P. Thostrup, A. Rønnau, C. Africh, E. Laegsgaard, J.K. Nørskov and F. Besenbacher, Phys. Rev. Lett., 2003, 90, 026101
247 A. Sanchez, S. Abbet, U. Heiz, W.D. Schneider, H. Häkkinen, R.N. Barnett and U. Landman, J. Phys. Chem. A, 1999, 103, 9573

248 Z. Yang, R. Wu and D.W. Goodman, Phys. Rev. B, 2000, 61, 14066

249 L. Giordano, G. Pacchioni, T. Bredow and J. Fernández Sanz, Surf. Sci., 2001, 471, 21

250 A. Vijay, G. Mills and H. Metiu, J. Chem. Phys., 2003, 118, 6536

251 Y. Wang and G.S. Hwang, Surf. Sci., 2003, 542, 72

252 J.A. Rodriguez, G. Liu, T. Jirsak, J. Hrbek, Z. Chang, J. Dvorak and A. Maiti, J. Am. Chem. Soc., 2002, 124, 5242

253 N. Lopez and J.K. Nørskov, Surf. Sci., 2002, 515, 175

254 Z. Yang, R. Wu, Q. Zhang and D.W. Goodman Phys. Rev. B, 2002, 65, 155407

255 A. Vittadini and A. Selloni, J. Chem. Phys., 2002, 117, 353

256 L. Zhang, F. Cosandey, R. Persaud and T.E. Madey, Surf. Sci., 1999, 439, 73

257 K. Højrup Hansen, T. Worren, S. Stempel, E. Laegsgaard, M. Baumer, H.J. Freund, F. Besenbacher and I. Stensgaard, Phys. Rev. Lett., 1999, 83, 4120

258 F. Cosandey, L. Zhang and T.E. Madey, Surf. Sci., 2001, 474, 1

259 F. Cosandey, and P. Stadelmann, Mas. Res. Soc. Symp. Proc., 2001, 589, 235

260 T. Akita, K. Tanaka, S. Tsubota and M. Haruta, Journal of Electron Microscopy, 2000, 49, 657

261 A. Renou and M. Gillet, Surf. Sci., 1981, 106, 27

262 S. Giorgio, C.R. Henry, B. Pauwels and G. Van Tendeloo, Materials Science and Engineering A, 2000, 297, 197

263 O. Dulub, W. Hebenstreit and U. Diebold, Phys. Rev. Lett., 2000, 84, 3646

264 M. Bowker, P. Stone, R. Bennett and N. Perkins, Surf. Sci., 2002, 497, 155

265 C. Xu, W.S. Oh, G. Liu, D. Y. Kim and D.W. Goodman, J. Vac. Sci. Technol. A, 1997, 15, 1261

266 J.R. Kitchin, M.A. Barteau and J.G. Chen, Surf. Sci., 2003, 526, 323

267 C.E.J. Mitchell, A. Howard, M. Carney and R.G. Egdell, Surf. Sci., 2001, 490, 196

268 P. Wynblatt and N.A. Gjostein, Acta Metallurgica, 1976, 24, 1165

269 P. Wynblatt, Acta Metallurgica, 1976, 24, 1175

270 P. Buffat, and J.P. Borel, Phys. Rev. A, 1976, 13, 2287

271 X. Lai and D.W. Goodman, J. Mol. Catal. A: Chem., 2000, 162, 33

272 X. Lai, T. P. St. Clair, and D. W. Goodman, Faraday Discuss., 1999, 114, 279

273 E.A. Willneff, C. Klanner and S.L. M. Schroeder, Chem. Commun., 2003, 258

274 R.A. Bennett, P. Stone and M. Bowker, Catal. Lett., 1999, 59, 994

275 A. Kolmakov, and D.W. Goodman, Catal. Lett., 2000, 70, 93

276 A. Kolmakov, and D.W. Goodman, Surf. Sci., 2001, 490, L597

277 K. Fukui, S. Sugiyama, and Y. Iwasawa, Phys. Chem. Chem. Phys., 2001, 3, 3871

278 E.C.H. Sykes, F.J. Williams, M.S. Tikhov and R.M. Lambert, J. Phys. Chem. $B, 2002,106,5390$

279 J.G. Allpress and J.V. Sanders, Surf. Sci., 1967, 7, 1

280 H. Poppa, K. Heinemann, and A.G. Elliot, J. Vac. Sci. Technol., 1971, 8, 471 
281 E.H. Lee, H. Poppa and G.M. Pound, Thin Solid Films, 1976, 32, 229

282 H. Sato, S. Shinozaki and L.J. Cicotte, J. Vac. Sci. Technol., 1969, 6, 62

283 A.K. Green, J. Dancy and E. Bauer, J. Vac. Sci. Tech., 1970, 7, 159

284 J.J. Metois, K. Heinemann and H. Poppa, Applied Physics Letters, 1976,

29, 134

285 J.J. Metois, K. Heinemann and H. Poppa, Philosophical Magazine, 1977, 35, 1413

286 J.J. Metois, K. Heinemann and H. Poppa, Thin Solid Films, 1977, 41, 197

287 J.M. Cowley and K.D. Neumann, Surf. Sci., 1984, 145, 301

288 K. Heinemann, H.K. Kim, and H. Poppa, J. Vac. Sci. Technol., 1979, 16, 622

289 S. Ferrero, A. Piednoir and C.R. Henry, Nanoletters, 2001, 1, 227

290 S. Giorgio, C. Chapon, C.R. Henry, G. Nihoul and J.M. Penisson, Philosophical Magazine A, 1991, 64, 87

291 K. Højrup-Hansen, S. Ferrero and C.R. Henry, Appl. Surf. Sci., accepted

292 P.M. Ajayan and L.D. Marks, Phys. Rev. Lett., 1989, 63, 279

293 J.L. Robins and T.N. Rodin, Surf. Sci., 1964, 2, 346

294 M. Kubo, R. Muira, R. Yamauchi, R. Vetrivel and A. Miyamoto, Appl. Surf. SCi., 1995, 89, 131

295 J.A. Venables and J.H. Harding, Journal of Crystal Growth, 2000, 211, 27

296 J.L. Robins, T.N. Rhodin and R.L. Gerlach, J. Appl. Phys., 1966, 37, 3893

297 T. Kizuka and N. Tanaka, Phys. Rev. B, 1997, 56, 10079

298 B. Pauwels, G. Van Tendeloo, W. Bouwen, L. Theil Kuhn, P. Lievens, H. Lei and M. Hou, Phys. Rev. B, 2000, 62, 10383

299 K. Blick, T.D. Mitrelias, J.S.J. Hargreaves, G.J. Hutchings, R.W. Joyner, C.J. Kiely and F.E. Wagner, Catal. Lett., 1998, 50, 211

300 K. Luo, D.Y. Kim and D.W. Goodman, J. Mol. Catal. A, 2001, 167, 191

301 C. Winkler, A. Carew, R. Raval, J. Ledieu and R. McGrath, Surf. Rev. Lett., 2001, 8, 693

302 S. Shaikhutdinov, R. Meyer, M. Naschitzki, M. Bäumer and H.J. Freund, Catal. Lett., 2003, 86, 211

303 J. Carrey, J.L. Maurice, F. Petroff and A. Vaurès, Phys. Rev. Lett., 2001, 86, 4600

304 J. Carrey, J.L. Maurice, F. Petroff and A. Vaurès, Surf. Sci., 2002, 504, 75

305 M. Ritter, W. Ranke and W. Weiss, Phys. Rev. B, 1998, 57, 7240

306 W. Weiss and W. Ranke, Progr. Surf. Sci., 2002, 70, 1

307 S. Shaikhutdinov, R. Meyer, D. Lahav, M. Bäumer, T. Klüner and H.-J. Freund, Phys. Rev. Lett., 2003, 91, 076102

308 N.G. Condon, F.M. Leibsle, T. Parker, A.R. Lennie, D.J. Vaughn and G. Thornton, Phys. Rev. B, 1997, 55, 15885

309 S.K. Shaikhutdinov, M. Ritter, X.G. Wang, H. Over and W. Weiss, Phys. Rev. B, 1999, 60, 11062

310 M. Haruta, T. Kobayashi, S. lijima and F. Delannay, Proc. 9th, Int. Cong. Catal., 1988, 3, 1206

311 C. Binns, Surf. Sci. Rep., 2001, 44, 1

312 G. Schmid, Chem. Rev., 1992, 92, 1709

313 U. Kreibig and L. Genzel, Surf. Sci., 1985, 156, 678

314 U. Kreibig, Solid State Communications, 1978, 28, 767

315 R. Monot, A. Châtelain and J.P. Borel, Phys. Lett. A, 1971, 34, 57

316 R. Dupree, C.T. Forwood and M.J.A. Smith, Physica Status Solidi, 1967, 24, 525

317 A.G. Shastri, A.K. Dayte and J. Schwank, J. Catal., 1984, 87, 265

318 S.J. Tauster and S.C. Fung, J. Catal., 1978, 55, 29
319 R. Kubo, Journal of the Physical Society of Japan, 1962, 17, 975

320 W.P. Halperin, Rev. of Modern Physics, 1986, 58, 533

321 C.P. Vinod, G.U. Kulkarni and C.N.R. Rao, Chem. Phys. Lett., 1998, 289, 329

322 C.N. Rao, V. Vijayakrishnan, H.N. Aiyer, G.U. Kulkarni and G.N. Subbanna, J. Phys. Chem., 1993, 97, 11157

323 P. Claus, A. Brückner, C. Mohr and H. Hofmeister, J. Am. Chem. Soc., 2000, 122, 11430

324 S.B. DiCenzo, S.D. Berry and E.H. Hartford, Phys. Rev. B, 1988, 38, 8465

325 S.T. Lee, G. Apai, M.G. Mason, R. Benbow and Z. Hurych, Phys. Rev. B, 1981, 23, 505

326 M.M. Alvarez, J.T. Khoury, T.G. Schaaff, M.N. Shafigullin, I. Vezmar and R.L. Whetten, J. Phys. Chem. B. 1997, 101, 3706

327 L. Stievano, S. Santucci, L. Lozzi, S. Calogero and F.E. Wagner, Journal of Non-Crystalline Solids, 1998, 232, 644

328 H.G. Boyen, T. Herzog, G. Kästle, F. Weigl, P. Ziemann, J.P. Spatz, M. Möller, R. Wahrenberg, M.G. Garnier and P. Oelhafen, Phys. Rev. B, 2002, 65, 075412

329 M.E. Lin, R. Reifenberger, A. Ramachandra and R.P. Andres, Phys. Rev. B, 1992, 46, 15498

330 P.H. Citrin and G.K. Wertheim, Phys. Rev. B, 1983, 27, 3176

331 P.H. Citrin, G.K. Wertheim and Y. Baer, Phys. Rev. Lett., 1978, 41, 1425

332 C.D. Wagner and J.A. Taylor, J. Electron Spectrosc. Relat. Phenom., 1982, 28, 211

333 M.G. Mason, Phys. Rev. B, 1983, 27, 748

334 K.S. Liang, W.R. Salaneck and I.A. Aksay, Solid State Communications, 1976, 19, 329

335 G.K. Wertheim, S.B. DiCenzo and S.E. Youngquist, Phys. Rev. Lett., 1983, 51, 2310

336 K.S. Kim, and N. Winograd, Chem. Phys. Lett., 1975, 30, 91

337 I. Coulthard, S. Degen, Y.J. Zhu and T.K. Sham, Canadian Journal of Chemistry, 1998, 76, 1707

338 O. Cordes, and M. Harsdorff, Appl. Surf. Sci., 1988, 33, 152

339 H. Roulet, J.M. Mariot, G. Dufour, and C.F. Hague, Journal of Physics F: Condensed Matter, 1980, 10, 1025

340 E. Costanzo, G. Faraci, A.R. Pennisi, S. Ravesi, A. Terrasi and G. Margaritondo, Solid State Communications, 1992, 81, 155

341 D. Dalacu, J.E. Klemberg-Sapieha and L. Martinu, Surf. Sci., 2001, 472, 33

342 S. Zafeiratos and S. Kennou, Surf. Sci., 1999, 443, 238

343 B. Richter, H. Kuhlenbeck, H.J. Freund and P. S. Bagus, Phys. Rev. Lett., accepted

344 A. Balerna, E. Bernieri, P. Picozzi, A. Reale, S. Santucci, E. Burattini and S. Mobilio, Phys. Rev. B, 1985, 31, 5058

345 C. Solliard and M. Flueli, Surf. Sci., 1985, 156, 487

346 A. Balerna, E. Bernieri, P. Picozzi, A. Reale, S. Santucci, E. Burattini and S. Mobilio, Surf. Sci., 1985, 156, 206

347 A. Pinto, A.R. Pennisi, G. Faraci, G. D’Agostino, S. Mobilio and F. Boscherini, Phys. Rev. B, 1995, 51, 5315

348 W. Vogel, D.G. Duff and A. Baiker, Langmuir, 1995, 11, 401

349 C. Chusuei, X. Lai, K. Luo and D.W. Goodman, Topics in Catalysis, 2001, 14,71 
350 Z. Yang and R. Wu, Phys. Rev. B, 2003, 67, 081403

351 J. Radnik, C. Mohr and P. Claus, Phys. Chem. Chem. Phys., 2003, 5, 172

352 A. Howard, D.N.S. Clark, C.E.J. Mitchell R.G. Egdell and V.R. Dhanak, Surf. Sci., 2002, 518, 210

353 N. Nilius, N. Ernst and H.J. Freund, Phys. Rev. B, 2002, 65, 115421

354 N. Nilius, N. Ernst and H.J. Freund, Surf. Sci., 2001, 478, L327

355 M. Haruta, S. Tsubota, A. Ueda, and H. Sakurai, Studies in Surface Science And Catalysis, 1993, 77, 45

356 K. Fukushima, G. H. Takaoka, J. Matsuo, and I. Yamada, Japanese Journal of Applied Physics, 1997, 36, 813

357 Y. Yuan, A. P. Kozlova, K. Asakura, H. Wan, K. Tsai, and Y. Iwasawa, J. Catal., 1997, 170, 191

358 M. A. P. Dekkers, M. J. Lippits, and B. E. Nieuwenhuys, Catal. Today, 1999, 54, 381

359 J. D. Grunwaldt, C. Kiener, C. Wögerbauer, and A. Baiker, J. Catal., 1999, 181,223

360 M. Okumura, S. Nakamura, S. Tsubota, T. Nakamura, M. Azuma and M. Haruta, Catal. Lett., 1998, 51, 53

361 J.A. Rodriguez, S. Chaturvedi, M. Kuhn, J. van Eck, U. Diebold, P.S. Robbert, H. Geisler and C.A. Ventrice, J. Chem. Phys., 1997, 107, 9146

362 J.A. Rodriguez, M. Pérez, T. Jirsak and J. Evans, J. Chem. Phys.Lett., 2003, 378, 526

363 C.L. Bianchi, S. Biella, A. Gervasini, L. Prati and M. Rossi, Catal. Lett., 2003, 85, 91

364 L.I. llieva, D.H. Andreeva, A.A. Andreev and Thermochimica Acta, 1997, 292, 169

365 Q. Fu, A. Weber, and M. Flytzani-Stephanopoulos, Catal. Lett., 2001, 77, 87

366 D. Andreeva, T. Tabakova, L. Ilieva, A. Naydenov, D. Mehanjiev and M.V. Abrashev, Appl. Catal. A, 2001, 209, 291

367 D.R. Rainer, C. Xu, P.M. Holmblad and D.W. Goodman, J. Vac. Sci. Technol. A, 1997, 15, 1653

368 J. France and P. Hollins, J. Electron Spectrosc. Relat. Phenom., 1993, 64, 251

369 C. Winkler, A.J. Carew, S. Haq and R. Raval, Langmuir, 2003, 19, 717

370 C. Lemire, R. Meyer, S. Shaikhutdinov and H.-J. Freund, Angew. Chemie Int. Ed., 2003, 43, 118

371 C. Lemire, R. Meyer, S. Shaikhutdinov and H.-J. Freund, Surf. Sci., 2004, 552,27

372 D.C. Meier and D.W. Goodman, J. Am. Chem. SoC., 2004, 126, 1892

373 D. Guillemot, V.Y. Boresko, V.B. Kazansky, M. Polisset-Thfoin and J. Fraissard, J. Chem. Soc. Faraday Trans., 1997, 93, 3587

374 M. Manzoli, A. Chorino and F. Boccuzzi, Surf. Sci., 2003, 532, 377

375 H. Liu, A.I. Kozlov, A.P. Kozlova, T. Shido, K. Asakura and Y. Iwasawa, J. Catal., 185 (1999) 252

376 M.A.P. Dekkers, M.J. Lippits and B.E. Nieuwenhuys, Catal. Lett., 1998, 56, 195

377 H. Liu, A.I. Kozlov, A.P. Kozlova, T. Shido, and Y. Iwasawa, Phys. Chem. Chem. Phys., 1999, 1, 2851

378 S. Minicò, S. Scirè, C. Crisafulli, A.M. Visco and S. Galvagno, Catal. Lett., 1997, 47, 273

379 L. Fan, N. Ichikuni, S. Shimazu, and T. Uematsu, Appl. Catal. A, 2003, 246, 87
380 F. Boccuzzi, G. Cerrato, F. Pinna and G. Strukul, J. Phys. Chem. B., 1998, 102, 5733

381 T.M. Salama, T. Shido, R. Ohnishi and M. Ichikawa, J. Phys. Chem., 1996, 100,3688

382 F. Boccuzzi, A. Chorino, M. Manzoli, P. Lu, T. Akita, S. Ichikawa and M. Haruta, J. Catal., 2001, 202, 256

383 D. Andreeva, Gold Bull., 2002, 35, 82

384 W.S. Epling, G.B. Hoflund, J.F. Weaver, S. Tsubota and M. Haruta, J. Phys. Chem., 1996, 100, 9929

385 M. Okumura, K. Tanaka, A. Ueda, and M. Haruta, Solid State Ionics, 1997, 95, 143

386 Y.J. Zhu, A. Schnieders, J.D. Alexander, and T.P. Beebe, Langmuir, 2002, 18,5728

387 K.H. Choi, B.Y. Coh and H.I. Lee, Catal. Today, 1998, 44, 205

388 V.A. Bondzie, S.C. Parker and C.T. Campbell, Catal. Lett., 1999, 63, 143

389 G. Martra, L. Prati, C. Manfredotti, S. Biella, M. Rossi and S., Coluccia, J. Phys. Chem. B, 2003, 107, 5453

390 T.S. Kim, J.D. Stiehl, C.T. Reeves, R.J. Meyer and C.B. Mullins, J. Am. Chem. Soc., 2003, 125, 2018

391 J.D. Stiehl, T.S. Kim, C.T. Reeves, R.J. Meyer and C.B. Mullins, J. Amer. Chem. Soc., 2003, 125, 2018

392 J.D. Stiehl, T.S. Kim, S.M. McClure and C.B. Mullins, J. Amer. Chem. Soc., 2004, 126, 1606

393 J.D. Stiehl, T.S. Kim, S.M. McClure, C.B. Mullins, in preparation

394 M. Valden, S. Pak, X. Lai and D.W. Goodman, Catal. Lett., 1998, 56, 7

395 G.R. Bamwenda, S. Tsubota, T. Nakamura and M. Haruta, Catal. Lett., 1997, 44, 83

396 T. Akita, P. Lu, S. Ichikawa, K. Tanaka and M. Haruta, Surface and Interface Analysis, 2001, 31, 73

397 G.C. Bond, Surf. Sci. 1985, 156, 966

398 R. Burch, The Royal Society Specialist Report on Catalysis, 1985, 7, 149

399 Y. lizuka, T. Tode, T. Takao, K. Yatsu, T. Takeuchi, S. Tsubota and M. Haruta, J. Catal., 1999, 187, 50

400 B. Schumacher, V. Plzak, M. Kinne, and R.J. Behm, Catal. Lett., 2003, 89, 109

401 F. Porta, L. Prati, M. Rossi, S. Coluccia and G. Martra, Catal. Today, 2000, 61, 165

402 C. Bianchi, F. Porta, L. Prati and M. Rossi, Topics in Catalysis, 2000, 13, 231

403 M.A. Bollinger and M.A. Vannice, Appl. Catal. B Environmental, 1996, 8, 417

404 U. Heiz, A. Sanchez, S. Abbet and W.D. Schneider, Chem. Phys., 2000, 262, 189

405 U. Heiz and W.-D. Schneider, J. Phys. D: Appl. Phys., 2000, 33, R85

406 U. Heiz, A. Sanchez, S. Abbett, and W.-D. Schneider, Eur. Phys. J. D, 1999, 9, 35

407 H. Häkkinen, S. Abbet, A. Sanchez, U. Heiz and U. Landman, Angew. Chemie Int. Ed., 2003, 42, 1297

408 C.C. Chusuei, X. Lai, K.A. Davis, E.K. Bowers, J.P. Fackler and D.W. Goodman, Langmuir, 2001, 17, 4113

409 J. Guzman and B.C. Gates, Nanoletters, 2001, 1, 689

410 W.D. Knight, K. Clemenger, W.A. de Heer, W.A. Saunders, M.Y. Chou and M. L. Cohen, Phys. Rev. Lett., 1984, 52, 2141 
411 W. Vogel, D.A.H. Cunningham, K. Tanaka, and M. Haruta, Catal. Lett., 1996, 40, 175

412 D.A.H. Cunningham, W. Vogel, H. Kageyama, S. Tsubota and M. Haruta, J. Catal., 1998, 177, 1

413 D.A.H. Cunningham, W. Vogel, R.M. Torres Sanchez, K. Tanaka and M. Haruta, J. Catal., 1999, 183, 24

414 G. Schmid, R. Pfeil, R. Boese, F. Bandermann, S. Meyer, G.H.M. Calis and J.W.A. van der Velden, Chem. Ber., 1981, 114, 3634

415 M.A. Marcus, M.P. Andrews, J. Zegenhagen, A.S. Bommannavar and P. Montano, Phys. Rev. B, 1990, 42, 3312

416 D. van der Putten and R. Zanoni, Phys. Lett. A, 1995, 208, 345

417 H. Zhang, G. Schmid and U. Hartmann, Nanoletters, 2003, 3, 305

418 H.G. Boyen, G. Kästle, F. Wiegl, B. Koslowski, C. Dietrich, P. Ziemann, J.P. Spatz, S. Riethmüller, C. Hartmann, M. Möller, G. Schmid, M.G. Garnier and P. Oelhafen, Science, 2002, 297, 1533

419 J.D. Grundwaldt, M. Maciejewski, O.S. Becker, P. Fabrizioli and A. Baiker, J. Catal., 1999, 186, 458

420 H. Berndt, I. Pitsch, S. Evert, K. Struve, M.M. Pohl, J. Radnik and A. Martin, Appl. Catal. A, 2003, 244, 169

421 M. Maciejewski, P. Fabrizioli, J.D. Grunwaldt, O.S. Becker and A. Baiker, Phys. Chem. Chem. Phys., 2001, 3, 3846

422 A.K. Tripathi, V.S. Kamble, and N.M. Gupta, J.Catal. 1999, 187, 332

423 C.K. Chang, Y.J. Chen and C. Yeh, Appl.Catal.A, 1998, 174, 13

424 S. Tsubota, T. Nakamura, K. Tanaka and M. Haruta, Catal. Lett., 1998, 56, 131

425 B.S. Upshade, T. Akita, T. Nakamura and M. Haruta, J. Catal., 2002, 209, 331

426 A. Wolf and F. Schüth, Appl. Catal. A, 2002, 226, 1

427 M. Haruta, A. Ueda, S. Tsubota, and R.M. Torres Sanchez, Catal. Today, 1996, 29, 443

428 R. Zanella, S. Giorgio, C.R. Henry and C. Louis, J. Phys. Chem. B, 2002, 106, 7634

429 S. Tsubota, D. Cunningham, Y. Bando and M. Haruta, Studies in Surface Science and Catalysis, 1993, 77, 325

430 D. Wang, Z.Hao, D. Cheng, X. Shi and C. Hu, J. Mol. Catal. A, 2003, 200, 229

431 R.J.H. Grisel, P.J. Kooyman and B.E. Niewenhuys, J. Catal, 2000, 191, 430

432 S.J. Lee and A. Gavriilidis, J. Catal., 2002, 206, 305

433 L. Prati and G. Martra, Gold Bull., 1999, 32, 96

434 J.N. Lin and B.Z. Wan, Appl.Catal.B: Env., 2003, 41, 83

435 C. Mohr, H. Hofmeister and P. Claus, J.Catal. 2003, 213, 86

436 C. Mohr, H. Hofmeister, J. Radnik and P. Claus, J. Am. Chem. Soc., 2003, 125, 1905

437 F. Boccuzzi, A. Chiorino and M. Manzoli, Surf. Sci. 2000, 454, 942

438 F. Boccuzzi, A. Chiorino, M. Manzoli, D. Andreeva and T. Tabakova, J. Catal., 1999, 188, 176

439 W.N. Delgass, M. Boudart and G. Parravano, J. Phys. Chem., 1968, 72, 3563

440 M. Batista-Leal, J.E. Lester and C.A. Lucchesi, Journal of Electron Spectroscopy and Related Phenomena, 1977, 11, 333

441 I.W. Bassi, F.W. Lytle and G. Parravano, J. Catal., 1976, 42, 139
442 G. Cocco, S. Enzo, G. Fagherazzi, L. Schiffini, I.W. Bassi, G. Vlaic, S. Galvagno and G. Parravano, J. Phys. Chem., 1979, 83, 2527

443 H. Kageyama, N. Kamijo, T. Kobayashi and M. Haruta, Physica B, 1989, 158, 183

444 J.Y. Lee and J. Schwank, J. Catal., 1986, 102, 207

445 M.M. Mohamed and M. Ichikawa, J. Colloid Interface Sci., 2000, 232, 381

446 A.M. Visco, F. Neri, G. Neri, A. Donato, C. Milone and S. Galvagno, Phys Chem Chem Phys., 1999, 1, 2869

447 E.D. Park and J.S. Lee, J. Catal., 1999, 186, 1

448 Z. Hao, L. An, H. Wang and T. Hu, Reaction Kinetics and Catalysis Letters, 2000, 70, 153

449 Y. Kobayashi, S. Nasu, S. Tsubota and M. Haruta, Hyperfine Interactions, 2000, 126, 95

450 T.M. Salama, R. Ohnishi, T. Shido and M. Ichikawa, J. Catal., 1996, 162, 169

451 T.M. Salama, T. Shido, H. Minagawa and M. Ichikawa, J. Catal., 1995, 152,322

452 T.M. Salama, T. Shido, R. Ohnishi and M. Ichikawa, Chem. Comm., 1994, 2749

453 R.D. Waters, J.J. Weimer and J.E. Smith, Catal. Lett., 1995, 30, 181

454 J. Guzman and B.C. Gates, Angew. Chemie, Int. Ed., 2003, 42, 690

455 Y.M. Kang, and B.Z. Wan, Appl. Catal. A 1995, 128, 53

456 R.M. Finch, N.A. Hodge, G.J. Hutchings, A. Meagher, Q.A. Pankhurst, M.R.H. Siddiqui, F.E. Wagner and R. Whyman, Phys Chem Chem Phys, 1999, 1, 485

457 N.A. Hodge, C.J. Kiely, R. Whyman, M.R.H. Siddiqui, G.J. Hutchings, Q.A. Pankhurst, F.E. Wagner, R.R. Rajaram, and S.E. Golunski, Catal. Today, 2002, 72, 133

458 Y.M. Kang and B.Z. Wan, Catal. Today, 1995, 26, 59

459 C. Sze, E. Gulari and B.G. Demczyk, Materials Letters, 1998, 36, 11

460 Q.Fu, H. Saltsburg and M. Flytzani-Stephanopoulos, Science, 2003, 301, 935

461 Q.Fu, S. Kudriavtseva, H. Saltsburg and M. Flytzani-Stephanopoulos, Chem. Eng. J., 2003, 93, 41

462 S. Schimpf, M. Lucas, C. Mohr, U. Rodemerck, A. Brückner, J. Radnik, H. Hofmeister and P. Claus, Catal. Today, 2002, 72, 63

463 H. Kageyama, S. Tsbubota, K. Kadono, K. Fukumi, T. Akai, N. Kamijo and M. Haruta, Journal de Physique IV, 1997, 7, 935

464 D. Horváth, L. Toth and L. Guczi, Catal. Lett., 2000, 67, 117

465 V. Idakiev, L. Ilieva, D. Andreeva, J.L. Blin, L. Gigot and B.L. Su, Appl. Catal. A, 2003, 243, 25

466 N.M. Gupta and A.K. Tripathi, J. Catal., 1999, 187, 343

467 S.K. Tanielyan and R.L. Augustine, Applied Catalysis A, 1992, 85, 73

468 L. Guczi, D. Horváth, Z. Pászti, L. Tóth, Z. E. Horváth, A. Karacs and G.Petõ, J. Phys. Chem. B, 2000, 104, 3183

469 A.Y. Stakheev and L.M. Kustov, Appl. Catal. A, 1999, 188, 3

470 J.D. Grunwaldt and A. Baiker, J. Phys. Chem. B, 1999, 103, 1002

471 Z.M. Liu and M.A. Vannice, Catal. Lett., 1997, 43, 51

472 L. Guczi, D. Horváth, Z. Pászti and G. Petõ, Catal. Today, 2002, 72, 101

473 F. Boccuzzi, A. Chorino, S. Tsubota and M. Haruta, J. Phys. Chem., 1996, 100,3625 
474 L.M. Molina and B. Hammer, Phys. Rev. Lett., 2003, 90, 206102

475 H.M. Ajo, V.A. Bondzie and C.T. Campbell, Catal. Lett., 2002, 78, 359

476 C. Yang, M. Kalwei, F. Schüth and K. Chao, Appl. Catal. A, 2003, 254, 289

477 W.S. Eppling, C.H.F. Peden, M.A. Henderson and U. Diebold, Surf. Sci., 1998, 412, 333

478 G. Pacchioni and A.M. Ferrari, Catal. Today, 1999, 50, 533

479 M.A. Henderson, W.S. Epling, C.L. Perkins, C.H.F. Peden and U. Diebold, J. Phys. Chem. B, 1999, 103, 5328

480 T.T. Magkoev, D. Rosenthal, S.L.M. Schröder and K. Christmann, Technical Physics Letters, 2000, 26, 894

481 Y. lizuka, H. Fujiki, N. Yamauchi, T. Chijiwa, S. Arai, S. Tsubota and M. Haruta, Catal. Today, 1997, 36, 115

482 A.M. Venezia, V. La Parola, G. Deganello, B. Pawelec and J.L.G. Fierro, J. Catal., 2003, 215, 317

483 L. Guczi, A. Beck, D. Horváth, Z. Koppány, G. Stefler, K. Frey, I. Sajó, O. Geszti, D. Bazin and J.Lynch, J. Mol. Catal. A, 2003, 204, 545

484 A. Venugopal, J. Aluha and M.S. Scurrell, Catal. Lett., 2003, 90, 1

485 Y. lizuka, K. Akita, A. Kawamoto, M. Date, S. Tsubota, M.Okumura and M. Haruta, submitted

486 K. Tanaka, Surf. Sci., 1996, 357, 721

487 R.J.H. Grisel and B.E. Nieuwenhuys, J. Catal, 2001, 199, 48

488 A. Eichler and J. Hafner, Surf. Sci., 1999, 433, 58

489 U. Burghaus and H. Conrad, Surf. Sci., 1996, 352, 253

490 Y.J. Chen and C. Yeh, J. Catal., 2001, 200, 59

491 W.A. Bone and G.W. Andrew, Proceedings of the Royal Society A, 1925, 109, 409

492 S.L.M. Schroeder, personal communication

493 A.G. Daglish and D.D. Eley, Proc. 2nd, Int. Cong. Catal. Paris 1960, 1961, 2, 1615

494 M. Olea, M. Kunitake, T. Shido and Y. Iwasawa, Phys. Chem. Chem. Phys., 2001, 3, 627

495 P. Mars, and D.W. van Krevelen, Chem. Eng. Sci. Spec. Suppl., 1954, 3, 41

496 N.M. Gupta and A.K. Tripathi, Gold Bulletin, 2001, 34, 120

497 W. Liu and M. Flytzani-Stephanopoulos, J. Catal., 1995, 153, 317

498 Z. Hao, L. Fen, G.Q. Luo, J. Liu, L. An and H. Wang, Appl. Catal. A, 2001, 213, 173

499 M. Okumura, J.M. Coronado, J. Soria, M. Haruta and J.C. Conesa, J. Catal., 2001, 203, 168

500 Y.M. Kang and B.Z. Wan, Catal. Today, 1997, 35, 379

501 A. Knell, P. Barnickel, A. Baiker and A. Wokaun, J. Catal., 1992, 137, 306

502 G.Y. Wang, W.X. Zhang, H.L. Lian, D.Z. Jiang and T.H. Wu, Appl. Catal. A, 2003, 239, 1

503 M.M. Schubert, V. Plzak, J. Garche and R.J. Behm, Catal. Lett., 2001, 76, 143

504 G.K. Bethke and H.H. Kung, Appl. Catal. A, 2000, 194, 43

505 F. Boccuzzi and A. Chorino, J. Phys. Chem. B, 2000, 104, 5414

506 G. Wang, W. Zhang, H. Lian, Q. Liu, D. Jhang and T. Wu, Reaction Kinetics and Catalysis Letters, 2002, 75, 343

507 D.A. Bulushev, L. Kiwi-Minsker, I. Yuranov, E.I. Suvorova, P.A. Buffat and A. Renken, J. Catal., 2002, 210, 149
508 D.A.H. Cunningham, W. Vogel and M. Haruta, Catal. Lett., 1999, 63, 43

509 M. Date and M. Haruta, J. Catal., 2001, 201, 221

510 M. Date Y. Ichihashi, T. Yamashita, A. Chiorino, F. Boccuzzi and M. Haruta, Catal. Today, 2002, 72, 89

511 H. Sakurai, A. Ueda, T. Kobayashi and M. Haruta, Chem. Comm., 1996, 271

512 H.H. Kung, M.C. Kung and C.K. Costello, J. Catal., 2003, 216, 425

513 C.K. Costello, J.H. Yang, H.Y. Law, Y. Wang, J.N. Lin, L.D. Marks, M. C. Kung and H. H. Kung, Appl. Catal. A, 2003, 243, 15

514 C.K. Costello, M.C. Kung, H.S. Oh, Y. Wang and H.H. Kung, Appl. Catal. A, 2002, 232, 159

515 H.S. Oh, J.H. Yang, C.K. Costello, Y. Wang, S.R. Bare, H.H. Kung and M.C. Kung, J. Catal., 2002, 210, 375

516 X.Q. Gong, P. Hu and R. Raval, J Chem. Phys., 2003, 119, 6324

517 J. Guzman, and B.C. Gates, J. Phys. Chem. B, 2002, 106, 7659

518 E.E. Strangland, K.B. Stevens, R.P. Andres and W.N. Delgass, J. Catal., 2000, 191, 332

519 Y.A. Kalvachev, T. Hayashi, S. Tsubota and M. Haruta, Studies in Surface Science and Catalysis, 1997, 110, 965

520 B.S. Upshade, S. Tsubota, T. Hayashi and M. Haruta, Chemistry Letters, 1998, 1277

521 T. Hayashi, K. Tanaka and M. Haruta, American Chemical Society 211th National Meeting Petroleum Division, 1996, 41, 71

522 M. Haruta, B.S. Upshade, S. Tsubota and A. Miyamoto, Res. Chem. Intermed., 1998, 24, 329

523 G. Mul, A. Zwijnenburg, B. van der Linden, M. Makkee and J.A. Moulijn, J. Catal., 2001, 201, 128

524 B.S. Upshade, Y. Yamada, T. Akita, T. Nakamura and M. Haruta, Appl. Catal. A, 2001, 215, 137

525 A.K. Sinha, S. Seelan, T. Akita, S. Tsubota and M. Haruta, Catal. Lett., 2003, 85, 223

526 A. Zwijnenburg, A. Goossens, W.G. Sloof, M.W. J. Crajé, A.M. van der Kraan, L.J. de Jongh, M. Makkee and J.A. Moulijn, J. Phys. Chem. B, 2002, 106, 9853

527 T.A. Nijhuis, B.J. Huizinga, M. Makkee and J.A. Moulijn, Industrial Engineering and Chemistry Research, 1999, 38, 884

528 P. Landon, P.J. Collier, A.J. Papworth, C.J. Kiely and G.J. Hutchings, Chem. Commun. 2002, 2058

529 M. Okumura, Y. Kitagawa, K. Yamaguchi, T. Akita, S. Tsubota and M. Haruta, Chem. Lett., 2003, 32, 822

530 P. Paredes Olivera, E. M. Patrito and H. Sellers, Surf. Sci., 1994, 313, 25

531 C. Sivadinarayana, T.V. Choudhary, L.L. Daemen, J. Eckert and D.W. Goodman, J. Amer. Chem. Soc., in press

532 M. Haruta, Studies in Surface Science and Catalysis, 2002, 145, 31

533 G.J. Hutchings, M.R.H. Siddiqui, A. Burrows, C.J. Kiely and R. Whyman, Journal of the Chemical Society Faraday Transcripts, 1997, 93, 187

534 S. Gardner, G.B. Hoflund, D.R. Schryer, J. Schryer, B.T. Upchurch and E.J. Kielin, Langmuir, 1991, 7, 2135

535 J.N. Lin, J.H. Chen, C.Y. Hsiao, Y.M. Kang and B.Z. Wan, Appl. Catal. B: Env., 2002, 36, 19 